\title{
NON-UNIFORM 3D ANTENNA ARRAY OPTIMAL CONFIGURATION VIA EXTREMUM SEEKING CONTROL
}

\author{
Dissertation \\ Submitted to \\ The School of Engineering of the \\ UNIVERSITY OF DAYTON \\ In Partial Fulfillment of the Requirements for \\ The Degree of \\ Doctor of Philosophy in Engineering \\ By \\ Mosa Aboabdalla Abdesalam \\ UNIVERSITY OF DAYTON \\ Dayton, Ohio \\ December, 2016
}




\section{NON-UNIFORM 3D ANTENNA ARRAY OPTIMAL CONFIGURATION VIA EXTREMUM SEEKING CONTROL}

Name: Abdesalam, Mosa Aboabdalla

\section{APPROVED BY:}

Raúl Ordóñez, Ph.D.

Advisor Committee Chairman

Professor, Department of Electrical and computer Engineering

John Lomis, Ph.D.

Committee Member

Professor, Department of Electrical and computer Engineering
Robert Penno, Ph.D.

Committee Member

Professor, Department of Electrical and computer Engineering
Muhammad Islam, Ph.D.

Committee Member

Professor, Department of Electrical and computer Engineering
Robert J. Wilkens, Ph.D, P.E.

Associate Dean for Research and Innovation Professor

School of Engineering
Eddy M. Rojas, Ph.D., MA., P.E.

Dean, School of Engineering 


\section{(c) Copyright by}

Mosa Aboabdalla Abdesalam

All rights reserved

2016 


\begin{abstract}
NON-UNIFORM 3D ANTENNA ARRAY OPTIMAL CONFIGURATION VIA EXTREMUM SEEKING CONTROL
\end{abstract}

Name: Abdesalam, Mosa Aboabdalla

University of Dayton

Advisor: Dr. Raúl Ordóñez

In this work, designing a system that controls a non-uniform antenna array will be attempted. This system will reconfigure the antenna array to find the position and orientation that produce the maximum objective (directivity). To create such a system, we will assume that we have $N$ vehicles carrying antennas. These vehicles will be in 1D, 2D, and 3D spaces (e.g., quad-copters). Each vehicle with its antenna has five degrees of freedom. The five degrees are as follows, three degrees for the position of the vehicle $(x, y, z)$, and two for the orientation of the antenna $(\theta, \phi)$. The system should be able to test every possible value for these five parameters to dynamically choose the position and orientation that maximize the directivity. By measuring the performance function value online, a sequence, generated by three Extremum Seeking Control (ESC) algorithms that are compared in this work, guides the regulator that drives the state of system to a set point that optimizes the performance function. This approach develops a novel method to achieve reconfigurable antenna arrays, by defining an objective function to be optimized on-line using three different ESC methods: (a) Perturbation-based Extremum Seeking Control (PESC), (b) Numerical Optimization-based Extremum Seeking Control Using Direct Search (NOESC-DS), and (c) Numerical Optimization-based 
Extremum Seeking Control Using Particle Swarm Optimization (NOESC-PSO). Simulation results are provided for confirming the validity and the effectiveness of the proposed methods. We also studied the sensitivity of PESC and NOESC-PSO to changes in the initial conditions of the antenna array. We have found ESC to be a viable and effective method to achieve reconfigurable antenna arrays, capable of satisfying on the fly a given performance objective, even when the array elements can be moved in 3D space and the orientations of the antenna elements are allowed to vary. This is a challenging scenario, and our results indicate that it could be feasibly solved in practice using the proposed approach. 
To my father

To my mother

To my wife and daughter

To all my brothers and sisters

To all those who have helped me along the way with their support

For without their support I would never have come this far. 


\section{ACKNOWLEDGMENTS}

First of all, I thank God for looking over me during these past years and for granting me the ability to complete this degree.

Growing up I would have never seen myself where I am today. The pursuit of an education after graduating college was not something I had planned. Going to a new country and having to learn the language and culture while also learning new things as a grad student was not the simplest decision. It was hard and there were times when I wanted to give up, but the people around me would not let me give up on myself. So I worked harder and I came out with a Masters degree, thinking that it would be the highest degree I would have. I spent three years with my family and getting used to what I had left behind before again I was encouraged to continue learning. One of many who pushed me was my late father, he saw me in a much higher place in life than I saw myself. I hope he is proud of me, and I wished he was here, but I know he is in a better place. Without him pushing me I may have never came to America, and I would not be where I am today. I would also have not met the amazing people I know.

I especially want to thank Professor Raúl Ordóñez for his guidance and advice. Prof. Ordóñez is a person I thrive to be like, and he has taught me much throughout the years. In the hardest moments of this study, he was there guiding and helping me by showing and teaching me things I would have never thought of. Prof. Ordóñez has much broad knowledge and talents, and is the kind of person that one meets once in a lifetime. He is a mentor that I am proud of having and I hope will be a teacher and friend in the years to come. 
There are also the committee members, Professor Robert Penno, Professor John Loomis, and Professor Muhammad Islam that I would like to thank for their time and advice, whether it is while I am presenting or just anytime they see me and give me advice about the things they know are worrying me. I would also like to thank the head of my department, Dr. Guru Subramanyam, who gave me a job at the university when I had financial problems.

I would also like to thank my friends who have been there for me and helped me through the small and big part of this study and of this life I have made in America. First Dr. Huthaifa Ahmad Al Issa, a friend I will always remember and cherish. He is the kind of person that would do anything for a friend, and he truly did that for me. Dr. Abdulmajid Mrebit is also someone who helped me even though he had his own things to worry about. There are also my friends in the non-linear lab with whom I spent many days with them in the past eight years. Especially Ouboti DjaneyeBoundjou, who was a dear and helpful friend during our time together. He is a kind soul that would help anyone in need, which is something I admire about him. Also Faraj Abdelhafeid and Esmail Abuhdima, who helped me with some of my greatest obstacles while working on this dissertation. To all my friends thank you for everything.

I would also like to thank my family in Libya. They have patiently waited for my homecoming for the past eight years. They encouraged me and pushed me to not come home until I am done. And when things were rough, they worked their hardest to make sure I do not run into any troubles here. Without them I would not know if I would have been able to stay and finish my degree or not. And finally I would like to deeply thank my wife and daughter. All my hard work is for them. I have the deepest respect and gratitude for them. They moved to a new country, learned a new language, a new culture, and continued to support me through it all. They reminded me of my origin and my culture and helped keep it alive, and made sure I kept in touch with my family and friends when I was so focused on this study that I forgot about other things. They helped me in 
ways that no words can describe and they are the reason why I work hard, so they get the life they deserve. I will forever be in their debt for all they have given up and done for me. I am where I am today because of them. 


\section{TABLE OF CONTENTS}

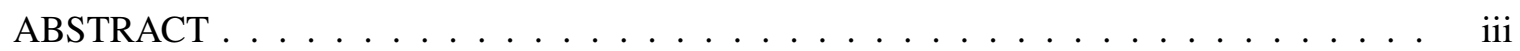

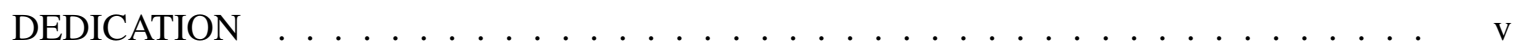

ACKNOWLEDGMENTS . . . . . . . . . . . . . . . . . . . . . vi

LIST OF FIGURES . . . . . . . . . . . . . . . . . . . . . . . . . xii

LIST OF TABLES $\ldots \ldots \ldots \ldots \ldots \ldots \ldots \ldots \ldots \ldots \ldots$. . . . . . . . . . . . . . . . . . . . . . . . .

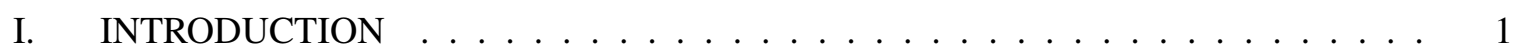

1.1 Motivation . . . . . . . . . . . . . . . . . . . . . 1

1.2 History and Literature Overview of Non-conformal Antenna Arrays . . . . . . . 2

1.3 History and Literature Overview of the Extremum Seeking Control . . . . . . . 4

1.4 History and Literature Overview of Particle Swarm Optimization . . . . . . . . 6

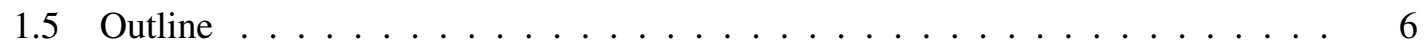

1.6 Perturbation-based Extremum Seeking Control . . . . . . . . . . . . . . . . 7

1.7 Numerical Optimization-based Extremum Seeking Control Using Direct Search 9

1.8 Numerical Optimization-based Extremum Seeking Control Using Particle Swarm Optimization . . . . . . . . . . . . . . . . . 10

II. PROBLEM STATEMENT . . . . . . . . . . . . . . . . . . . . . . . . . . . . . . . 13

2.1 Important Parameters of the Antenna $\ldots \ldots \ldots \ldots$

$2.1 .1 \quad$ Antenna Coordinate System . . . . . . . . . . . . . . . . 15

2.1 .2 Geometry Antenna Configuration . . . . . . . . . . . . . . . . . . . 16

2.1 .3 Radiation Pattern . . . . . . . . . . . . . . . . . . . . . . 16

2.1 .4 Directivity . . . . . . . . . . . . . . . . . . 17

2.2 Main Concepts of Short Dipole Antennas . . . . . . . . . . . . . . . . 18

2.3 Antenna Arrays . . . . . . . . . . . . . . . . . . . . . . . . . 21

2.4 Kinematic Point-Mass Model Simulations . . . . . . . . . . . . . . . . . . . 24 
III. 1D FIXED ANTENNA ORIENTATION . . . . . . . . . . . . . . . . . . . . . . . . 26

$3.1 \quad$ Two Vehicles in 1D with Fixed Antenna Orientation at $\left(\theta_{1,2}, \phi_{1,2}\right)=(0,0) \ldots$. . 26

$3.1 .1 \quad$ Perturbation-based Extremum Seeking Control . . . . . . . . . . . 26

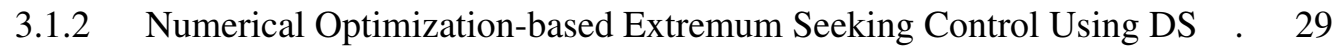

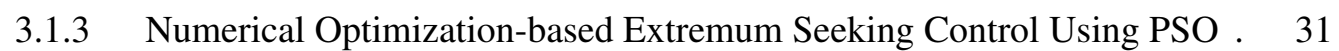

$3.1 .4 \quad$ Comparison Results of PESC and NOESC with DS and PSO . . . . . . 33

3.2 Three Vehicles in 1D with Fixed Antenna Orientation $\ldots \ldots \ldots \ldots$

3.2.1 Perturbation-based Extremum Seeking Control . . . . . . . . . . . . . . 35

3.2.2 Numerical Optimization-based Extremum Seeking Control Using DS . . 36

$3.2 .3 \quad$ Numerical Optimization-based Extremum Seeking Control Using PSO . 38

$3.2 .4 \quad$ Comparison Results of PESC and NOESC with DS and PSO . . . . . . 40

3.3 Sensitivity of Extremum Seeking Control to Changes the Initial Conditions in Antenna Array Applications . . . . . . . . . . . . . . . . . . . . 41

3.3.1 Perturbation-based Extremum Seeking Control at the Initial Condition

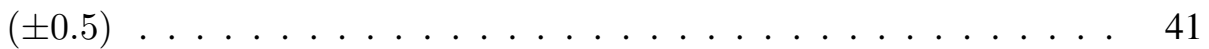

3.3.2 Numerical Optimization-based Extremum Seeking Control Using PSO at the Initial Condition $( \pm 0.5) \ldots \ldots \ldots$. . . . . . . . . . . 42

3.3.3 Perturbation-based Extremum Seeking Control at the Initial Condition $( \pm 7) 43$

3.3.4 Numerical Optimization-based Extremum Seeking Control Using PSO at the Initial Condition $( \pm 7) \ldots \ldots \ldots \ldots$. . . . . . . . . . 45

3.3.5 Perturbation-based Extremum Seeking Control With a different of Initial Conditions ...................... 46

3.3.6 Numerical Optimization-based Extremum Seeking Control Using PSO With a different of Initial Conditions $\ldots \ldots \ldots$. . . . . . . 48

3.3 .7 Conclusion for Sensitivity . . . . . . . . . . . . . . . . . . . 49

IV. 2D AND 3D FIXED ANTENNA ORIENTATION $\ldots \ldots \ldots \ldots \ldots$

$4.1 \quad$ Three Vehicles in 2D with Fixed Antenna Orientation $\ldots \ldots \ldots$

$4.2 \quad$ 2D Arbitrary Positioned Antenna Arrays . . . . . . . . . . . . . . . . . . 51

$4.2 .1 \quad$ Using Perturbation-based Extremum Seeking Control . . . . . . . . . . 51

4.2.2 Using Numerical Optimization-based Extremum Seeking Control with DS 55

$\begin{array}{lll}4.2 .3 & \text { Using Numerical Optimization-based Extremum Seeking Control with PSO } 57\end{array}$

$4.2 .4 \quad$ Comparison Results of PESC and NOESC with DS and PSO . . . . . . 59

4.3 Four Vehicles in 3D with Fixed Antenna Orientation $\ldots \ldots \ldots$

$4.3 .1 \quad$ Using Perturbation-based Extremum Seeking Control . . . . . . . . . . 61

4.3.2 Using Numerical Optimization-based Extremum Seeking Control with DS 64

4.3 .3 Comparison of Results of PESC and NOESC-DS . . . . . . . . . . . 66

V. $\quad$ 3D CASE WITH ARBITRARY ANTENNA ORIENTATION $\ldots \ldots \ldots \ldots$

$5.1 \mathrm{~N}$ Vehicles in 3D with Arbitrary Antenna Orientation $\ldots \ldots \ldots \ldots$ 
5.2 TWO Vehicles in 3D with Arbitrary Antenna Orientation . . . . . . . . . . . . 69

$5.2 .1 \quad$ Using Perturbation-based Extremum Seeking Control . . . . . . . . . . 69

5.2.2 Using Numerical Optimization-based Extremum Seeking Control with DS 74

\begin{tabular}{|lll}
\hline 5.2 .3 & Using Numerical Optimization-based Extremum Seeking Control with PSO 77
\end{tabular}

5.2.4 Comparison of PESC and NOESC with DS and PSO for 2 Vehicles in 3D 81

5.3 Four Vehicles in 3D with Arbitrary Antenna Orientation . . . . . . . . . . 81

5.3 .1 Using Perturbation-based Extremum Seeking Control . . . . . . . . . . 82

5.3.2 Using Numerical Optimization-based Extremum Seeking Control with DS 86

5.3 .3 Comparison of PESC and NOESC-DS for 4 Vehicles in 3D . . . . . . . 90

VI. CONCLUSIONS AND FUTURE WORK $\ldots \ldots \ldots \ldots \ldots \ldots$

6.1 Conclusions . . . . . . . . . . . . . . . . . . . . . . . . . . 91

6.2 Future Work $\ldots \ldots \ldots \ldots \ldots$. . . . . . . . . . . . . . . . . 92

BIBLIOGRAPHY . . . . . . . . . . . . . . . . . . . . . . . . 94 


\section{LIST OF FIGURES}

$1.1 \quad$ Perturbation-based extremum seeking control. . . . . . . . . . . . . . . . . . 9

1.2 Numerical optimization-based extremum seeking control using DS. . . . . . . . . 10

$1.3 \quad$ Numerical optimization-based extremum seeking control using PSO. . . . . . . . . . 11

$2.1 \quad$ Antenna coordinate system. . . . . . . . . . . . . . . . . 15

2.2 Antenna geometry . . . . . . . . . . . . . . . . . . . 16

2.3 Radiation pattern lobes $\ldots \ldots \ldots \ldots \ldots \ldots$

2.4 Short dipole antenna geometry . . . . . . . . . . . . . . . . . . . . 18

2.5 Three-dimensional radiation pattern of short dipole. . . . . . . . . . . . . . 20

2.6 Cross-section of field pattern in $y z, x z$, and $x y$ planes. $\ldots \ldots \ldots \ldots \ldots$

2.7 Geometry of a two-element short dipole arrays . . . . . . . . . . . . . . 22

2.8 Geometry of a two-element array positioned along the $x$-axis $\ldots \ldots \ldots \ldots$

2.9 Directivity versus element spacing. . . . . . . . . . . . . . . . . . . 24

2.10 Two vehicles in $1 \mathrm{D}$ and fixed orientation of antenna $\ldots \ldots \ldots$

$3.1 \quad$ Objective function for PESC in a 1D case (2 vehicles). . . . . . . . . . . . . . 27

3.2 Position for PESC in a 1D case $(2$ vehicles $) . \ldots \ldots \ldots \ldots$

3.3 Control input of $x$-axis for PESC in a 1D case (2 vehicles). . . . . . . . . . . . 28 
$3.4 \quad$ Objective function for NOESC-DS in a 1D case (2 vehicles). . . . . . . . . . . . . 29

3.5 Position for NOESC-DS in a $1 \mathrm{D}$ case $(2$ vehicles $) . \ldots \ldots \ldots \ldots$

3.6 Control input of $x$-axis for NOESC-DS in a 1D case (2 vehicles). . . . . . . . . . . 30

$3.7 \quad$ Objective function for NOESC-PSO in a 1D case (2 vehicles). . . . . . . . . . 31

$3.8 \quad$ Position for NOESC-PSO in a 1D case $(2$ vehicles $) . \ldots \ldots \ldots \ldots \ldots$

$3.9 \quad$ Control input of $x$-axis for NOESC-PSO in a 1D case (2 vehicles). . . . . . . . . . 32

3.10 Three vehicles in 1D and fixed orientation antenna. . . . . . . . . . . . . . 34

3.11 Objective function for PESC in a 1D case (3 vehicles). . . . . . . . . . . . . 35

3.12 Position for PESC in a $1 \mathrm{D}$ case $(3$ vehicles). . . . . . . . . . . . . 36

3.13 Control input of $x$-axis for PESC in a 1D case (3 vehicles). . . . . . . . . . . . 36

3.14 Objective function for NOESC-DS for 1D case (3 vehicles). . . . . . . . . . . 37

3.15 Position for NOESC-DS in a 1D case (3 vehicles). . . . . . . . . . . . . 37

3.16 Control input of $x$-axis for NOESC-DS in a 1D case (3 vehicles). . . . . . . . . . 38

3.17 Objective function for NOESC-PSO in a 1D case (3 vehicles). . . . . . . . . . . . 39

3.18 Position for NOESC-PSO in a 1D case (3 vehicles). . . . . . . . . . . . . . 39

3.19 Control input of $x$-axis for NOESC-PSO in a 1D case (3 vehicles). . . . . . . . . . 40

3.20 Objective function for PESC for 1D case (3 vehicles). . . . . . . . . . . . . 41

3.21 Position for $\mathrm{PESCl}$ for $1 \mathrm{D}$ case $(3$ vehicles). . . . . . . . . . . . . . 42

3.22 Objective function for NOESC-PSO in a 1D case (3 vehicles). . . . . . . . . . . . 43

3.23 Position for NOESC-PSO in a 1D case (3 vehicles). . . . . . . . . . . . . . 43

3.24 Objective function for PESC in a 1D case (3 vehicles). . . . . . . . . . . . . 44 
3.25 Position for PESC in a $1 \mathrm{D}$ case $(3$ vehicles $) . \ldots \ldots \ldots$. . . . . . . . . . . 44

3.26 Objective function for NOESC-PSO in a 1D case (3 vehicles). . . . . . . . . . . . 45

3.27 Position for NOESC-PSO in a 1D case (3 vehicles). . . . . . . . . . . . . . . 46

3.28 Set of objective function for PESC in a 1D case (3 vehicles). . . . . . . . . . . . 47

3.29 Set of position for PESC in a 1D case (3 vehicles). . . . . . . . . . . . . . . 47

3.30 Set of objective function for NOESC-PSO in a 1D case (3 vehicles). . . . . . . . . 48

3.31 Set of position for NOESC-PSO in a 1D case (3 vehicles). . . . . . . . . . . . . 49

4.1 Three vehicles in 2D and fixed antenna orientation . . . . . . . . . . 51

4.2 Objective function for PESC in a 2D case (3 vehicles). . . . . . . . . . . . 52

4.3 Position for PESC in a 2D case (3 vehicles). . . . . . . . . . . . . . . . . 53

4.4 Control input of $x$-axis for PESC in a 2D case (3 vehicles). . . . . . . . . . . . 54

4.5 Vehicles trajectory for PESC in a 2D case (3 vehicles). . . . . . . . . . . . 54

$4.6 \quad$ Objective function for NOESC-DS in a 2D case (3 vehicles). . . . . . . . . . . . 55

$4.7 \quad$ Position for NOESC-DS in a $2 \mathrm{D}$ case $(3$ vehicles $) . \ldots \ldots \ldots \ldots$

4.8 Control input of $x$-axis for NOESC-DS in a 2D case (3 vehicles). . . . . . . . 56

$4.9 \quad$ Vehicles trajectory for NOESC-DS in a 2D case (3 vehicles). . . . . . . . . . . 57

4.10 Objective function for NOESC-PSO in a 2D case (3 vehicles). . . . . . . . . . 58

4.11 Position for NOESC-PSO in a 2D case (3 vehicles). . . . . . . . . . . . 58

4.12 Control input of $x$-axis for NOESC-PSO in a 2D case (3 vehicles). . . . . . . . . . 59

4.13 Vehicles trajectory for NOESC-PSO in a 2D case (3 vehicles). . . . . . . . . . 59

4.14 Four vehicles in 3D and fixed antenna orientation . . . . . . . . . . . . . 61 
4.15 Objective function for PESC in a 4 vehicles in 3D. $\ldots \ldots \ldots \ldots$

4.16 Position for PESC in a 4 vehicles in 3D. . . . . . . . . . . . . . 63

4.17 Control input for PESC in a 4 vehicles in 3D. . . . . . . . . . . . . . . 63

4.18 Trajectory for for PESC in a 4 vehicles in 3D. . . . . . . . . . . . . . . . 64

4.19 Objective function for NOESC-DS for 3D case (4 vehicles). . . . . . . . . . . 65

4.20 Position for NOESC-DS for 3D case (4 vehicles). . . . . . . . . . . . . . 65

4.21 Control input of $x$-axis for NOESC-DS for 3D case (4 vehicles). . . . . . . . . . 66

4.22 Vehicles trajectory for NOESC-DS for 3D case (4 vehicles). . . . . . . . . . . 66

5.1 Geometry of N vehicles in 3D for non-uniformly spaced and orientation ntenna array 69

5.2 Objective function of two vehicles in 3D antenna orientated (PESC). . . . . . . . 71

$5.3 \quad$ Optimal positions of two vehicles in 3D antenna orientated (PESC). . . . . . . . . 71

5.4 Optimal angles of two vehicles in 3D antenna orientated (PESC). . . . . . . . . 72

5.5 Control input for positions of two vehicles in 3D antenna orientated (PESC). . . . $\quad 72$

5.6 Control input for angles of two vehicles in 3D antenna orientated (PESC). . . . . . 73

5.7 Vehicle trajectory for two vehicles in 3D antenna orientated (PESC). . . . . . . . 73

$5.8 \quad$ Objective function of two vehicles in 3D antenna orientated (NOESC-DS). . . . . . 74

5.9 Optimal positions of two vehicles in 3D antenna orientated (NOESC-DS). . . . . . 75

5.10 Optimal Angles of two vehicles in 3D antenna orientated (NOESC-DS). . . . . . . 75

5.11 Control input for positions of two vehicles in 3D antenna orientated (NOESC-DS). $\quad 76$

5.12 Control input for angles of two vehicles in 3D antenna orientated (NOESC-DS). . . 76

5.13 Vehicle trajectory for two vehicles in 3D antenna orientated (NOESC-DS). . . . . . 77 
5.14 Objective function of two vehicles in 3D antenna orientated (NOESC-PSO). . . . . 78

5.15 Optimal positions of two vehicles in 3D antenna orientated (NOESC-PSO). . . . . 79

5.16 Optimal angles of two vehicles in 3D antenna orientated (NOESC-PSO). . . . . . . 79

5.17 Control input for positions of two vehicles in 3D antenna orientated (NOESC-PSO). 80

5.18 Control input for angles of two vehicles in 3D antenna orientated (NOESC-PSO). . $\quad 80$

5.19 Vehicle trajectory for two vehicles in 3D antenna orientated (NOESC-PSO). . . . . 81

5.20 Objective function of four vehicles in 3D and antenna orientated (PESC). . . . . . 83

5.21 Optimal positions of four vehicles in 3D and antenna orientated (PESC). . . . . . . 84

5.22 Optimal orientation angles for the antenna for four vehicles in 3D (PESC). . . . . . 84

5.23 Control input for positions of four vehicles in 3D antenna orientated (PESC). . . . 85

5.24 Control input for angles of four vehicles in 3D antenna orientated (PESC). . . . . . 85

5.25 Vehicle trajectory for four vehicles in 3D antenna orientated (PESC). . . . . . . . . 86

5.26 Objective function of four vehicles in 3D antenna orientated (NOESC-DS). . . . . 87

5.27 Optimal position of four vehicles in 3D antenna orientated (NOESC-DS). . . . . . 87

5.28 Optimal angle of four vehicles in 3D antenna orientated (NOESC-DS). . . . . . . . 88

5.29 Control input for the position of four vehicles in 3D antenna orientated (NOESC-DS). 88

5.30 Control input for the angles of four vehicles in 3D antenna orientated (NOESC-DS). 89

5.31 Vehicle trajectory for four vehicles in 3D antenna orientated (NOESC-DS). . . . . 89 


\section{LIST OF TABLES}

3.1 Comaparison results of three algorithms for Case 2 Vehicles . . . . . . . . . . . . 34

3.2 Comparison results of three algorithms for Case 3 Vehicles $\ldots \ldots \ldots$. . . . . . . 40

4.1 Comparison results of three algorithms in a 2D case (3 vehicles). . . . . . . . . . 60 


\section{CHAPTER I}

\section{INTRODUCTION}

\subsection{Motivation}

Non-conformal antenna arrays are complex and difficult to design and analyze, and it is desirable to create a system that is able to autonomously reconfigure itself to achieve a desired objective in real time, or close to real time. Many previous researches have handled circuit array antennas, planar antennas, and linear antenna arrays. By taking advantage of the straightforward mathematical basics of these types of antennas, researchers were able to optimize different systems. These antenna arrays could be considered relatively less complex and easier to work with compared to the non-conformal antenna arrays. Non-conformal antenna arrays were a subject of study, before this work, using algorithms such as genetic algorithms and particle swarm optimization algorithms. Their accomplished results were fairly satisfying. This work is an attempt to go farther with non-conformal antennas by giving them five degrees of freedom. These five degrees of freedom will give the ability to adjust the position and orientation of the antennas. We propose to use Extremum Seeking Control (ESC) algorithm, for the first time, to create a system that is able to autonomously reconfigure itself to achieve some desired objective. Three main methods are used to achieve this purpose, Perturbation-based Extremum Seeking Control (PESC) method, Numerical Optimization-based Extremum Seeking Control Using Direct Search (NOESC-DS) method and Numerical Optimization-based Extremum Seeking Control Using Particle Swarm Optimization 
(NOESC-PSO). This work is an attempt to go farther with non-conformal 1D, 2D and $3 \mathbf{D}$ antenna arrays by giving the ability to adjust the position and orientation of the antenna to give the highest directivity with unknown objective function, position and orientation.

\subsection{History and Literature Overview of Non-conformal Antenna Arrays}

An antenna is a conductor by which radio waves are radiated out or received. Radio receivers and transmitters require antennas to couple the electrical connection to the electromagnetic field to carry out the radiation process. The signals are carried through the air (or space) by electromagnetic waves (i.e., radio) at the speed of light with almost no transmission loss. To convey signals (i.e., information) in mobile phones, televisions, radio systems, and data networks radio waves are used. Broad applications of radio waves are not limited to technology measurements such as radar, radio astronomy and Global Positioning System (GPS). The most important parameters of the antenna design are directivity, gain, bandwidth, mutual coupling, and radiation pattern. For example, a low-gain antenna receives or transmits over a wide range while a high directivity antenna increases signal strength. Several antennas that are connected and arranged in some specific structure to form a single antenna are called antenna arrays. One of the main antenna array features is the ability to produce combined radiation patterns, which is not the case in single antennas [1, 2].

The significance of directive antennas was realized, when the arrival of radio communications came about. In 1937 Wolff [3] showed how to determine the radiating system that would be built to produce a specified directive characteristic. In 1943 Schelkunoff [4] issued his paper on the mathematical theory of linear arrays that used a synthesis technique, which is independent of the nature of Wolff's theory. Dolph (1946) utilized the equal-ripple property of Chebyshev polynomials to optimize the main beam and the level of the sidelobes [5]. In the following decade, based on the

addition of terms of the $\frac{\sin (\theta)}{\theta}$ type, Woodward and Lawson created a synthesis method [6, 7]. As 
defined by Dolph, Taylor (1955) showed how to determine the continuous- current excitation function which would have the optimal property [8]. Taylor showed how the ideal equal-sidelobe pattern could be obtained arbitrarily closely, because beforehand realizability limitations prevented a realistic solution. All the previous work was made to enhance the uniform array elements. The first articles on improving array performance via geometry optimization date back to the early 1960s. Unz [9] studied linear arrays in 1960 and noted that performance improvement could be obtained by holding the weights constant and varying the element positions. An advantage of the methods of synthesizing an antenna array having the distribution of its elements is that they offer more degrees of freedom to the designer by what Sahalos [10] and Unz [11] say. In 1960, King, Packard, and Thomas [12] proposed eliminating grating lobes via element placement in an array. Later in 1961, Harrington [13] considered small element perturbations in an attempt to synthesize the desired array pattern. For use in radio astronomy, Lo suggest using a non-uniformly spaced antenna array based on what they discovered in 1961 [14]. In 1995, Lan, Jeffers, and Boucher presented the successive approximation method and dynamic programming method for the unequally spaced arrays [15]. Even with the help of computers, solving the problem analytically for large arrays is still very complicated by what the consensus of research today has shown. The problem is a highly non-linear problem, and the solution is not as mathematically convenient for periodic antenna arrays. Many optimization algorithms have proven to be successful tools for solving these electromagnetic problems [16, 17, 18], providing good results in a reasonable amount of time, which makes approaching it as an optimization problem the most fitting way to solve. An analytical technique for synthesis of unequally spaced arrays with linear, planar, cylindrical and spherical geometry was presented by Kummar and Branner in 2005 [19].

By taking advantage of the Genetic Algorithm (GA) and the Particle Swarm Optimization (PSO) to help search in the solution space for an optimal design, in whichever way they decide to define 
it, researchers would have approached the problem as an optimization problem. In 2008, a nonuniform circular antenna array using PSO was designed by Najjar and Khodier [20]. To reduce the elements space and amplitude perturbation, Abdolee and Vakilian developed a simple and fast GA in 2008 [21]. In order to minimize sidelobe levels in wideband arrays, Bevelacqua and Balanis, using the PSO algorithm with optimal weights, determined optimal array geometries in 2009 [22]. In 2011, Ridwan, Abdo, and Jorswicck designed non-uniform antenna arrays using GA, and Rupcic, Mandric, and Zagar learned the reduction of sidelobes by non-uniform elements spacing of a spherical antenna array [23, 24]. In 2012, Lager and Simeoni, and Coman presented mutual coupling in non-uniform array antennas in a very effective way, and they proposed the radiation properties of non-uniform array antennas [25, 26].

\subsection{History and Literature Overview of the Extremum Seeking Control}

In this section, we first present a historical development of ESC started in [27] and then review the existing literature, especially for the last decades. ESC was a point of interest in the 1950s and 1960s [28, 29, 30, 31, 32] especially by Russian researchers. At that time, it was the main subject in many books, including Tsien [33] (1954), Feldbaum [34] (1959), Krasovskii [35] (1964), Wilde [36], and Chinaev [37] (1969). Later in the 1980s Sternby proposed the adaptive control was introduced and became more popular among researchers. ESC did not gain popularity again until the 1990s as a real-time optimization tool. It can also be considered as an adaptive non linear control method. The research neglection of the ESC after the 1960s, which continued for three decades, was caused by two reasons. One reason was the evolution of computers, which caused many researchers in the real-time optimization to pursue the development of general-purpose optimization algorithms. Another reason was the fact that adaptive control objectives started to distinguish the stabilization from the optimization and this is where model reference adaptive control algorithms shine. At that time, the ESC approach of assuming any dynamic system as static caused it to be 
unattractive [38]. ESC has seen a resurgence after its stability analysis was established in Krstić and Wang [39] for continuous-time systems. And Ariyur and Krstic looked at real-time optimization by extremum-seeking control [40]. Krstić also studied the performance improvement and limitations in extremum seeking control [41]. Choi, Krstić, Ariyur, and Lee [42] published a work about discrete-time systems. Many successful applications of ESC have been reported in the literature, including particle beam matching [43], tokamak fusion devices [44], source seeking with nonholonomic unicycles [45, 46], control of combustion instability [47], maximizing the pressure rise in an axial flow compressor [48], limit cycle minimization [49], optimal positioning of mobile sensors under stochastic noise [50], control of thermoacoustic instability [51], and optical fiber amplifiers [52].

Guay and his team worked on the gradient-based extremum seeking control with adaptive design and they achieved some developments in [53], where they introduced the issue and the solution of ESC for a certain class of nonlinear systems with unknown parameters, where an explicit structure information for the performance function to be maximized is required. Zhang, and Ordóñez [54, 55, 56] present ESC and application of a numerical optimization-based extremum seeking control (NOESC) approach, utilizing more systematic studies, and then in [57, 58, 59], where the state regulation combines with the numerical optimization to make the ESC scheme. The book by Zhang and Ordóñez [27] presents extremum-seeking control and applications a numerical optimizationbased approach. However, ESC has never before been used in high-performance 1D, 2D and 3D antenna array systems. In this work we will be using the PESC and NOESC and as an optimization control for design of non-uniform $1 \mathbf{D}, 2 \mathbf{D}$ and $3 \mathbf{D}$ antenna arrays, in the arbitrary position and orientation. 


\subsection{History and Literature Overview of Particle Swarm Optimization}

Kennedy and Eberhart introduced the (PSO) in 1995 [60]. Kennedy and Eberharts version of PSO is now considered to be the Classic Algorithm, and the newer enhanced versions to be PSO. PSO is an idea derived from swarm behavior in birds flocking and fish schooling. Many afterwards worked to improve upon and use PSO in their own studies including [61, 62, 63, 64, 65]. Numerical optimization can be used with particle swarm optimization (PSO). From these studies different types of PSO are revealed, including, but not limited to, adaptive particle swarm optimization (APSO). Djaneye-Boundjou, Ordóñez and Gazi revealed the previously mentioned APSO by using Lyapunov's direct method to stabilize the optimizer [66], this technique is used in this study. In this study numerical optimization extremum seeking control using PSO is implemented. By measuring the performance function value online, a sequence, generated by PSO algorithm, guides the regulator that drives the state of system approaching to the set point that optimizes the performance. This study will provide the ability to adjust the position of the antennas by giving them one degree of freedom, while focusing and studying the initial condition. To create this system, that is able to autonomously and dynamically reconfigure itself, the ESC algorithm will be used to achieve a desired objective (directivity of the antenna array). The PESC method, NOESC with DS, and NOESC using PSO will be the three methods used to attempt this system.

\subsection{Outline}

The dissertation is set up in a way where Chapter One is an introduction on the history and workings of everything used in this study. Chapter Two is the problem statement. The important parameters of an antenna, including the geometry antenna configuration, radiation patterns, and directivity, the main concepts of a dipole antenna, antenna arrays, and the kinematic point-mass model system are laid out and explained. Continuing to Chapter Three, which is when the simulations that 
are made and studied are explained, antennas with fixed orientation in 1D space simulations are laid out. We start with two vehicles in 1D with fixed antenna orientation. Results given by PESC, NOESC with DS, and NOESC with PSO are shown and compared. Then three vehicles are tested in 1D space, and again the results of the three algorithms are explained and compared. The sensitivity of the two methods, PESC and NOESC with PSO, to initial condition is examined via simulations is also in Chapter Three. Afterworlds, Chapter Four is where simulation results for three and four vehicles with fixed antenna orientation in 2D and 3D using PESC and NOESC with PSO are given. The simulation results are compared. An explanation of arbitrary antennas is also given. Chapter Five studies arbitrary antenna orientation in 3D for two vehicles and four vehicles. Finally, Chapter Six is an overall summary of what is analyzed and learned in the study and what future studies may try to do to enhance on the information learned.

\subsection{Perturbation-based Extremum Seeking Control}

The idea behind PESC is that an estimate of the minimizer/maximizer of a map gets disturbed by a sinusoidal signal and by measuring the corresponding outputs, the estimate is adjusted. This estimate is then disturbed again and used as an input of the map. In this way, the estimate will approach the value of the minimizer/maximizer [40]. Figure 1.1] shows the basic PESC-scheme. This gradient estimate can be used by the steepest ascent controller to guide a vehicle to the peak of an objective function. The PESC estimation is advantageous for real-time applications where complex gradient calculations are not feasible. Additionally, PESC estimator does not require a continuous objective function model, which makes it ideal for unknown and noisy environments. Throughout this dissertation, we will deal with the general nonlinear system

$$
\begin{gathered}
\dot{x}=f(x, u), \\
y=J(x),
\end{gathered}
$$


where $x \in \mathbb{R}^{\mathrm{n}}$ is the state, $u \in \mathbb{R}$ is the input, $y \in \mathbb{R}$ is the performance output, and the function $f: D \times \mathbb{R} \rightarrow \mathbb{R}^{\mathrm{n}}$ and $J: D \rightarrow \mathbb{R}^{\mathrm{n}}$ are sufficiently smooth on $D \subseteq \mathbb{R}^{\mathrm{n}}$. For the simplification of analysis, we assume $D=\mathbb{R}^{\mathrm{n}}$ throughout the dissertation. This system is assumed to be stable or stabilizable at each of these equilibria by a local feedback controller. Suppose that we have a smooth control law

$$
u=\alpha(x, \theta)
$$

parameterized by a scalar parameter $\theta$. The closed-loop system.

$$
\dot{x}=f(x, \alpha(x, \theta)),
$$

then has equilibria parameterized by $\theta$. We make the following assumptions about the closed-loop system.

Assumption 1 There exists a smooth function $g: \mathbb{R} \rightarrow \mathbb{R}^{\mathrm{n}}$ such that

$$
f(x, \alpha(x, \theta))=0 \quad \text { if and only if } \quad x=g(\theta) .
$$

Assumption 2 For each $\theta \in \mathbb{R}$, the equilibrium $x=g(\theta)$ of the system 1.1 is locally exponentially stable.

Assumption 3 There exists $\theta^{*} \in \mathbb{R}$ such that

$$
\begin{aligned}
& (J \circ g)^{\prime}\left(\theta^{*}\right)=0, \\
& (J \circ g)^{\prime \prime}\left(\theta^{*}\right)<0 .
\end{aligned}
$$

Thus, we assume that the output equilibrium map

$$
y=J(g(\theta))
$$


has a maximum at $\theta=\theta^{*}$. Our objective is to develop a feedback mechanism that maximizes the steady state value of $y$ but without requiring knowledge of $\theta^{*}$ or the functions $J$ and $g$.

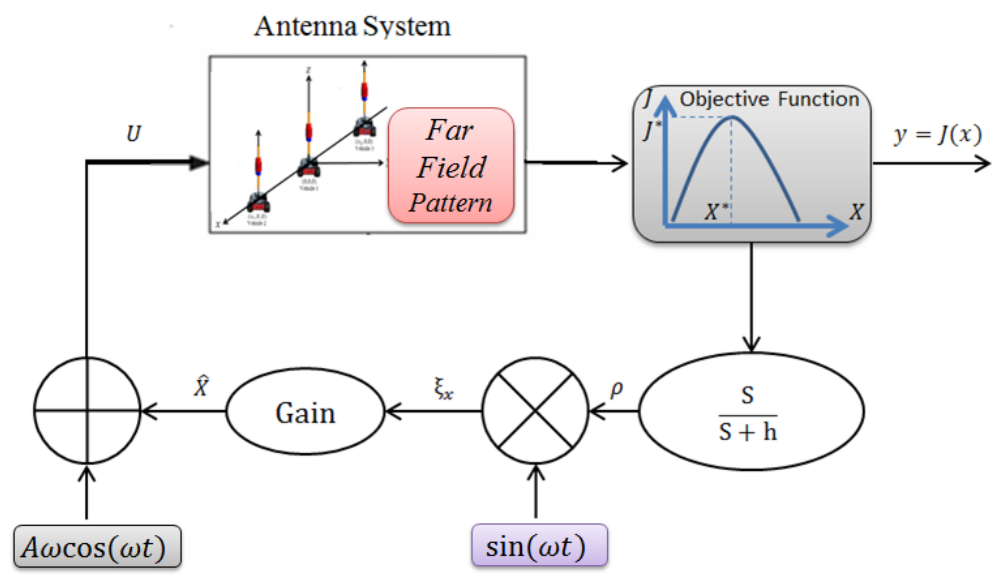

Figure 1.1: Perturbation-based extremum seeking control.

\subsection{Numerical Optimization-based Extremum Seeking Control Using Direct Search}

A block diagram of NOESC is proposed in Figure 1.3 , where the nonlinear system is modeled as the antenna system 1.1 and the performance function is the directivity of the antenna 1.2 . The extremum seeking controller (state regulator) ensures that the state $x$ travels along the set point sequence $x_{k}^{s}$ generated by the numerical optimization algorithm, which eventually converges to a maximizer of the performance function. The $x$ and $x_{k}^{s}$ are shown in the Figure 1.3 .

The basic NOESC algorithm [27] is as follows:

Step 0 Given $t_{0}=0$, let $x_{0}=x\left(t_{0}\right)$ and $k=0$. 
Step 1 Using an optimization algorithm, produce $x_{k+1}^{s}$ based on current state $x_{\left(t_{k}\right)}$, the measurements of $J\left(x_{\left(t_{k}\right)}\right), \nabla J\left(x\left(t_{k}\right)\right)$ or $\nabla^{2} J\left(x\left(t_{k}\right)\right)$. Denote

$$
x_{k+1}^{s}=O P T I M I Z E R\left(x_{\left(t_{k}\right)}\right) \text {. }
$$

Step 2 Design a state regulator $u$ that regulates the state $x_{t_{k}}$ to $x_{k+1}$ in a finite time $\delta_{k}$, let

$$
t_{k+1}=t_{k}+\delta_{k}
$$

Step 3 Set $k \leftarrow k+1$. Go to step 1 , and repeat.

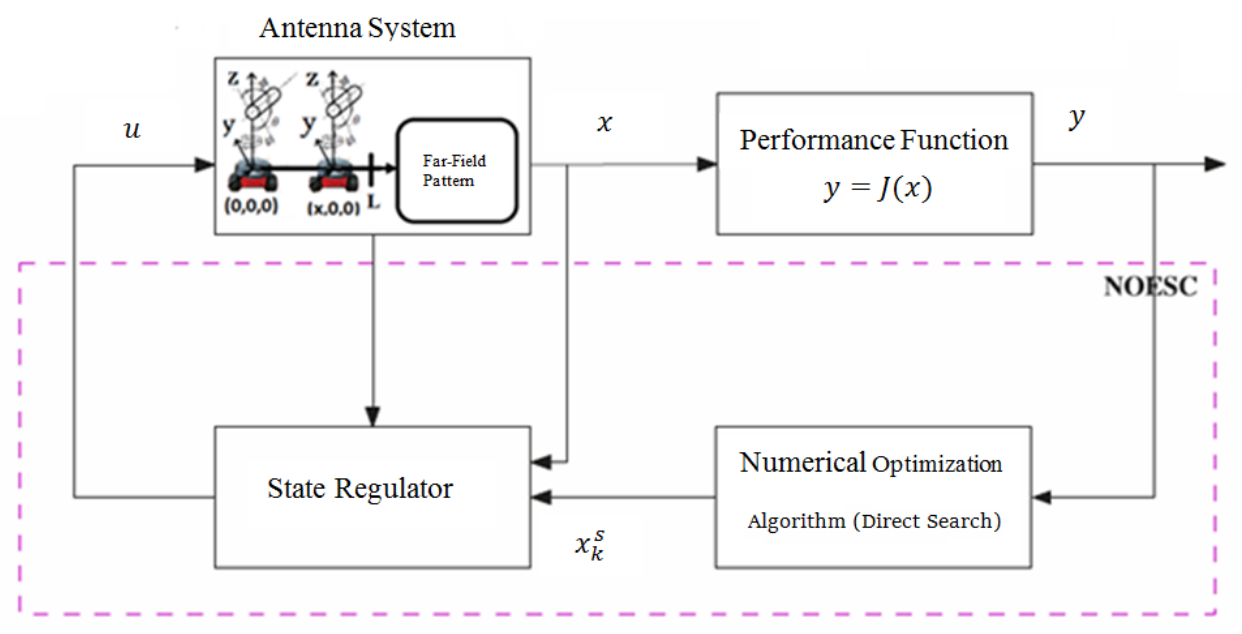

Figure 1.2: Numerical optimization-based extremum seeking control using DS.

\subsection{Numerical Optimization-based Extremum Seeking Control Using Particle Swarm Optimization}

Population based stochastic optimization method known as Particle Swarm Optimization (PSO) is encouraged from social interaction observed in bird flocking or fish schooling. It is an efficient optimization technique proved by experimental evidence [53, 64, 65]. As described in [66], the dynamics of particle $i$ are given by the equations 


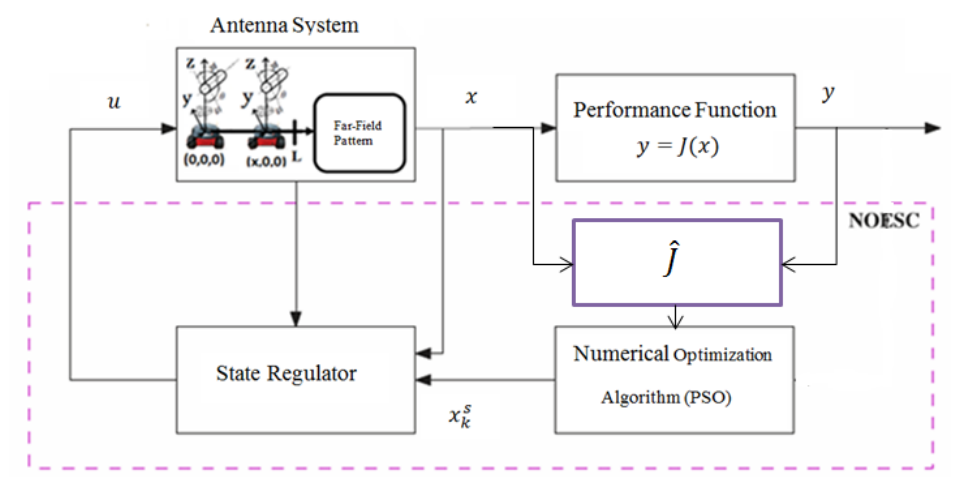

Figure 1.3: Numerical optimization-based extremum seeking control using PSO.

$$
\begin{aligned}
v^{i}(t+1)= & \left.w^{i} v^{i}(t)+\varphi_{1}^{i}(t)\left(p^{i}(t)-x^{i}(t)\right)\right) \\
& \left.+\varphi_{2}^{i}(t)\left(g^{i}(t)-x^{i}(t)\right)\right)
\end{aligned}
$$

$x^{i}(t+1)=x^{i}(t)+v^{i}(t+1)$,

where $i=1,2, \ldots, N$ and all product operations are performed element-wise. The variable $t$ denotes generation or iteration number. The position and velocity vectors of particle $i$ at generation $\mathrm{t}$ are respectively $x^{i}(t), v^{i}(t) \in \mathbb{R}^{\mathrm{n}}$. Moreover, $p^{i}(t), g^{i}(t) \in \mathbb{R}^{\mathrm{n}}$, respectively the personal best position achieved by particle $i$ and the global best position achieved by particle $i$ and its neighbors up to generation $t$, are given by

$$
\begin{aligned}
& p^{i}(t)=\arg \quad \max _{k=0, \ldots, i}\left\{\hat{J}\left(p^{i}(k)\right)\right\}, \\
& g^{i}(t)=\arg \max _{j \in S_{i}(k)}\left\{\hat{J}\left(p^{i}(k)\right)\right\}
\end{aligned}
$$

with $S_{i}(k)$ being the sets of all the neighbors of particle $i$ at generations $k=0, \ldots, t$. The parameter $w$ is referred to as inertia factor. The vectors $\left.\left.\varphi_{1}^{i}(t) \in\right] 0, \bar{\varphi}_{1}^{i}\right]^{n}$ and $\left.\left.\varphi_{2}^{i}(t) \in\right] 0, \bar{\varphi}_{2}^{i}\right]^{n}$ are independent 
identically uniformly distributed random vectors. We require that, for all $t, \varphi_{1}^{i}(t) \neq 0$ and $\varphi_{2}^{i}(t) \neq 0$ for reasons that will become clear later. We use a global neighborhood topology where all swarm particles are neighbors of one another. That means, for all $N$ swarm particles, $g^{1}(t)=g^{2}(t)=\ldots=$ $g^{N}(t)=g(t)$. It cannot be assumed that $J$ can be sampled at arbitrary locations. Only the location where the vehicles are is known. $J$ is only allowed to be sampled at the location of a vehicle. It makes sense to allow the vehicles to move. Only the samples from $J$ where the vehicles actually are can be used. As a vehicle moves from its current position to the next point in the sequence, given by the optimizer, $J$ is sampled along the way. The samples are used to construct a splines interpolation between those points. This is called interpolation $\hat{J} . \hat{J}$ is an approximation of $J$ that gets better as the vehicle collects more points. The PSO algorithm is run on $\hat{J}$, and it produces the next regulation point to go to from $\hat{J}$, as shown in Figure 1.3 The Adaptive Particle Swarm Optimization, which were results in Djaneye-Boundjou, Ordóñez and Gazi study [66] found by using Lyapunovs direct method to prove that our error system is ultimately uniformly bounded and hence the optimizer is stable, is implanted to advance results in this study. 


\section{CHAPTER II}

\section{PROBLEM STATEMENT}

Antenna arrays have been in use for some time, and it is well known that they offer distinct advantages over single radar antennas, including the ability to produce directed radiation patterns, flexible beam shaping, and an increase in bandwidth that would be difficult/expensive to obtain with a single antenna. The resulting radiation pattern of a radar array depends on the element pattern of each individual element, relative phase, and the physical spacing of the elements.

The most usual configurations are linear arrays, and planar arrays (linear arrays of linear arrays). Non-conformal arrays (those not limited to a plane, or not regularly arranged) have been and continue to be actively studied. There is a significant increase in complexity in non-conformal arrays and determining analytical solutions for the radiation pattern is difficult. Non-homogeneous arrays (where not all elements are of the same type) present similar challenges. But, like non-conformal arrays, they are attractive because of the flexibility they offer.

A very attractive type of radar array is one that is mobile. For example, a group of Unmanned Air Vehicles (UAVs) or Unmanned Ground Vehicles (UGVs) could be equipped with radar antennas on each vehicle. If the vehicles are constrained to move in formation, different array configurations can be achieved. Any number of vehicles can be accommodated to create a desired radiation pattern. The array can be dynamically reconfigured by either changing the vehicle formation (which 
can be maintained in a suitable two or three dimensional shape), or by changing the individual antenna magnitudes and phases, or both. Because the spacing between antennas can be dynamically modified, the frequency of the resulting antenna array can be increased or decreased, as needed. A high level of robustness can be potentially achieved, e.g., with respect to jamming, and even if some elements of the array fail or are destroyed.

To be truly effective, this kind of mobile sensor array must be able to reconfigure itself depending on the task at hand, or on environmental circumstances. The mobile radar sensor network will be subject to errors in positions for each UAV due to control and actuator limitations, wind, etc. In order to robustly maintain the desired beam shape of the overall array, closed-loop corrections of element magnitude and phase will probably be necessary.

Some research objectives can thus be conceived, including the development of a modeling and simulation tool for radar system design, and then the Investigation of formation control, position sensing, and phase control performance requirements needed to achieve robust beam shaping.

In this work, designing a system that controls a non-uniform antenna array will be attempted. This system will reconfigure the antenna to find the position and orientation that produce the maximum objective (directivity). To create such a system, we will assume that we have $N$ vehicles carrying antennas. These vehicles will be in 3D space (e.g., quadcopters). Each vehicle with its antenna has five degrees of freedom. The five degrees are as follows, three degrees for the position of the vehicle $(x, y, z)$, and two for the orientation of the antenna $(\theta, \phi)$. The system should be able to test every possible value for these five parameters to dynamically choose the position and orientation that maximize the directivity. To implement and optimize the system, we will use three algorithms, PESC, NOESC-DS and NOESC-PSO. 


\subsection{Important Parameters of the Antenna}

Many antenna parameters are defined in terms of the electromagnetic field radiated by the antenna. Finding the radiated fields, which can be done using an analytical approximation for the current distribution on the antenna together with a numerical method implemented in software is the first step in antenna analysis. The antenna radiation pattern can be obtained and various properties of the pattern, such as directivity and beam width, can be quantified once the radiated fields are known.

\subsubsection{Antenna Coordinate System}

Each array element location is represented by the antenna spherical coordinate system as shown in Figure 2.1. The angles $(\theta, \phi)$ refer to the azimuthal $(x-y)$ plane and elevation $(z)$ plane. The azimuthal plane is defined with respect to the $+x$-axis as the angle origin is rotated in the $x-y$ plane about the $z$-axis for $0<\phi<2 \pi(\mathrm{rad})$. The elevation plane is defined with respect to the $+z$-axis as the angle origin and is defined for $0<\theta<\pi$ (rad).

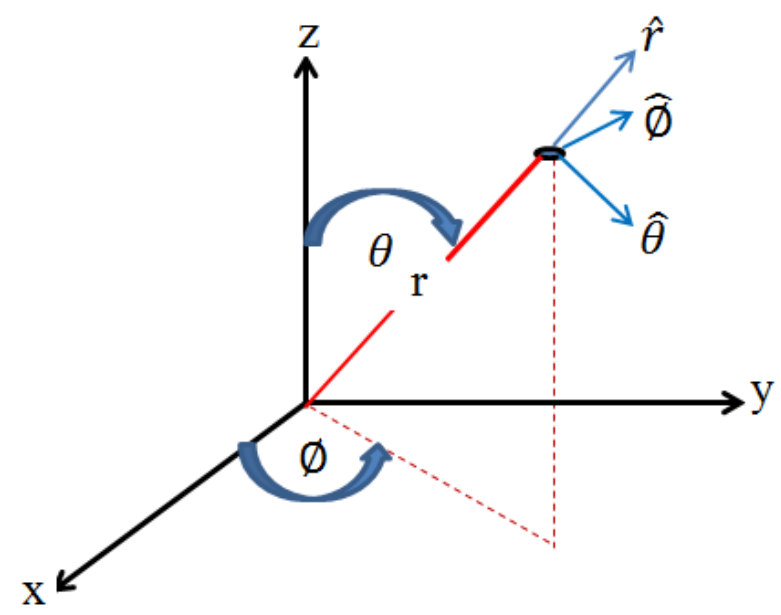

Figure 2.1: Antenna coordinate system. 


\subsubsection{Geometry Antenna Configuration}

The antenna array is a geometrical configuration of antenna elements grouped in such a way as to direct, or focus, radiated power towards a particular angular direction in space, enhancing spatial diversity of transmitted/received signals. The choice of geometry, number of elements, amplitude and phase of the array elements depends on the angular pattern desired. Typical geometric configurations are shown in Figure 2.2 below. Our attention in this work will be about $1 \mathbf{D}, 2 \mathbf{D}$ and $3 \mathbf{D}$ arbitrary antenna arrays.

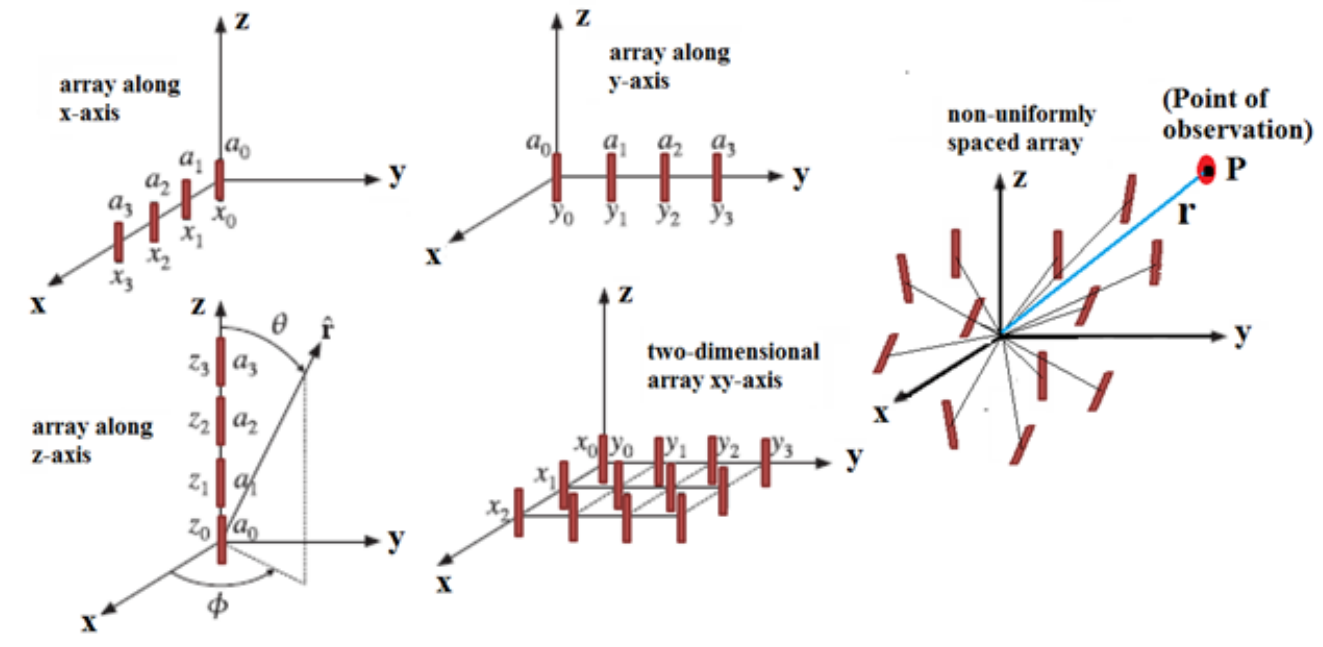

Figure 2.2: Antenna geometry

\subsubsection{Radiation Pattern}

A measure of power or radiation distribution with respect to a particular type of coordinates is the radiation pattern of an antenna. It is typically represented by a three dimensional graph, or polar plots of the horizontal and vertical cross sections. The pattern of an ideal isotropic antenna, which 
radiates equally in all directions, would look like a sphere. The antenna radiation pattern as shown in Figure 2.3]1].

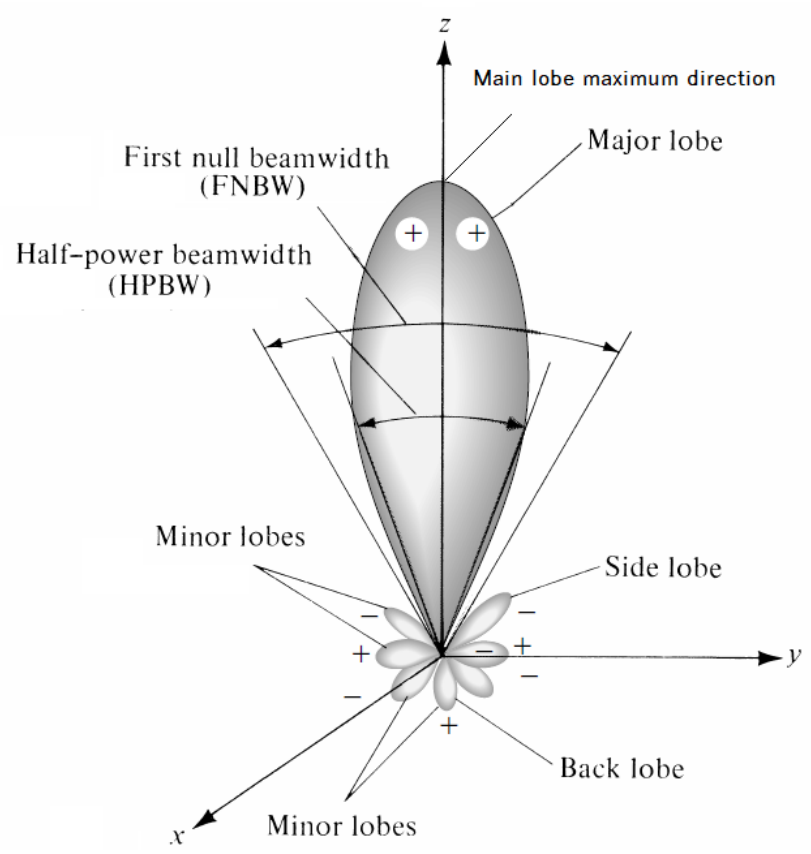

Figure 2.3: Radiation pattern lobes

\subsubsection{Directivity}

Directivity is defined as the ratio of the radiation intensity (radiated power per unit solid angle) in the direction of the main beam to the average radiation intensity. To put it another way, the directivity of an antenna or an array is the ratio of its maximum radiation intensity to the radiation intensity of an isotropic (omni directional) source' radiating the same total power. It measures the ability of concentrating the radiated energy in the main-beam direction. Optimal techniques using extremum seeking control are used to maximize the directivity of antenna arrays. 


\subsection{Main Concepts of Short Dipole Antennas}

The short dipole with length $L$ is oriented along the $z$-axis, as shown in Figure 2.4. The location of the dipoles are described by the coordination system where $(x, y, z)$ or, equivalently $(R, \theta, \phi)$ represent the observation point coordinates, $\left(x_{n}^{\prime}, y_{n}^{\prime}, z_{n}^{\prime}\right)$ or, equivalently $\left(R_{n}^{\prime}, \theta_{n}^{\prime}, \phi_{n}^{\prime}\right)$ represent the coordinates of the source, $R$ is the distance from any point on the source to the observation point, and $1 \leq n \leq N$ is associated with elements of the array [1, 2].

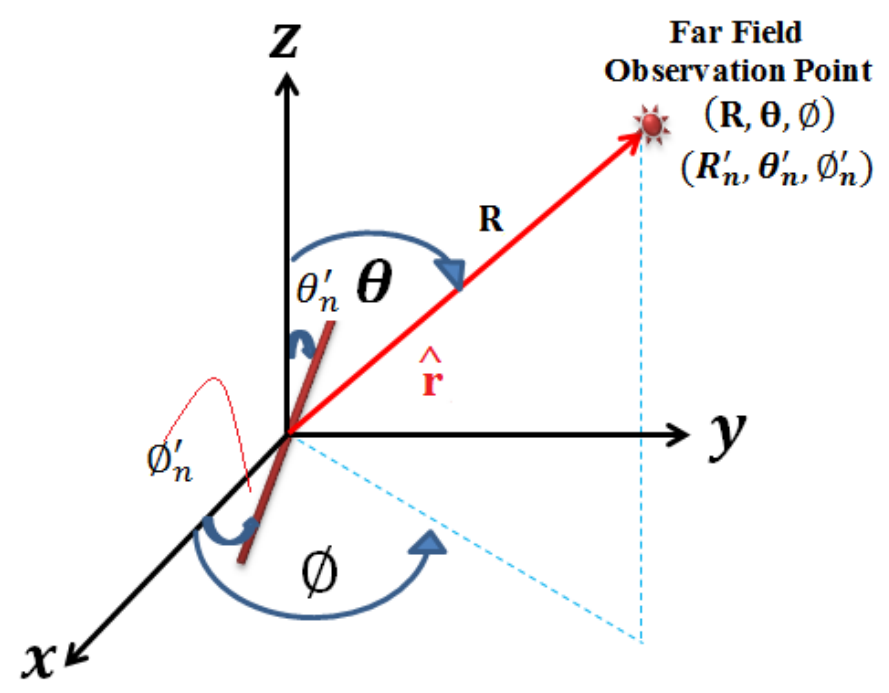

Figure 2.4: Short dipole antenna geometry.

The current that flows in the short dipole is given by

$$
I\left(z^{\prime}\right)=I_{0} \hat{z}^{\prime}
$$

where $\hat{z}^{\prime}$ is

$$
\hat{z}^{\prime}=\sin \theta_{n} \cos \phi_{n} \hat{x}+\sin \theta_{n} \sin \phi_{n} \hat{y}+\cos \theta_{n} \hat{z}
$$


for Arbitrarily oriented short dipole

$$
\bar{r}^{\prime}=z^{\prime} \hat{z}^{\prime}=z^{\prime}\left(\sin \theta_{n} \cos \phi_{n} \hat{x}+\sin \theta_{n} \sin \phi_{n} \hat{y}+\cos \theta_{n} \hat{z}\right)
$$

where the unit vector is

$$
\hat{r}=\sin \theta \cos \phi \hat{x}+\sin \theta \sin \phi \hat{y}+\cos \theta \hat{z} .
$$

Calculate the rotated element pattern along the $z$-axes follows as:

$$
\begin{aligned}
\hat{r} \cdot \bar{r}^{\prime} & =z^{\prime}\left(\sin \theta \cos \phi \sin \theta_{n} \cos \phi_{n}+\sin \theta \sin \phi \sin \theta_{n} \sin \phi_{n}+\cos \theta \cos \theta_{n}\right) \\
& =z^{\prime}\left(\sin \theta \sin \theta_{n}\left[\cos \phi \cos \phi_{n}+\sin \phi \sin \phi_{n}\right]+\cos \theta \cos \theta_{n}\right) \\
& =z^{\prime}\left(\sin \theta \sin \theta_{n} \cos \left(\phi-\phi_{n}\right)+\cos \theta \cos \theta_{n}\right)=\hat{z}^{\prime}\left(\hat{r} \cdot \bar{r}^{\prime}\right) .
\end{aligned}
$$

The vector potential is seen as

$$
\begin{aligned}
\overline{\mathrm{A}} & =\mu \int J\left(x^{\prime}, y^{\prime}, z^{\prime}\right) e^{\frac{-j \beta R}{4 \pi R}} d z^{\prime} \\
& =\mu \int I_{0} \hat{z}^{\prime} \frac{e^{-j \beta\left(r \cdot \bar{r}^{\prime} \cdot \hat{r}\right)}}{4 \pi R} d z^{\prime}=\mu\left(\frac{e^{-j \beta r}}{4 \pi R}\right) I_{0} \int \hat{z}^{\prime} e^{j \beta\left(\hat{r} \cdot \hat{r}^{\prime}\right) z^{\prime}} d z^{\prime},
\end{aligned}
$$

where $I_{0}=$ constant, and $\beta=2 \pi / \lambda$ is the wave number.

If we assume the incident wave is $\theta$ - polarized, the

$$
A_{\theta}=\hat{\theta} \cdot \overline{\mathrm{A}}=\mu I_{0} \frac{e^{-j \beta r}}{4 \pi r} \int\left(\hat{\theta} \cdot \hat{z}^{\prime}\right) e^{j \beta\left(\hat{r} \cdot \hat{r}^{\prime}\right) z^{\prime}} d z^{\prime}
$$

where $\hat{\theta}$ is

$$
\hat{\theta}=\hat{x} \cos \theta \cos \phi+\hat{y} \cos \theta \sin \phi-\hat{z} \sin \theta .
$$

The polarization of the incident wave is

$$
\begin{aligned}
\hat{\theta} \cdot \hat{z}^{\prime} & =\sin \theta_{n} \cos \phi_{n} \cos \theta \cos \phi+\sin \theta_{n} \sin \phi_{n} \cos \theta \sin \phi-\sin \theta \cos \theta_{n} \\
& =\sin \theta_{n} \cos \theta\left(\cos \phi_{n} \cos \phi+\sin \theta_{n} \sin \phi\right)-\sin \theta \cos \theta_{n} \\
& =\sin \theta_{n} \cos \theta\left(\cos \left(\phi-\phi_{n}\right)\right)-\sin \theta \cos \theta_{n} .
\end{aligned}
$$

We first find $A_{\theta}$ from equation 2.7 as follows:

$$
\begin{aligned}
A_{\theta} & =\mu I_{0} \frac{e^{-j \beta r}}{4 \pi r} \int_{-L / 2}^{L / 2}\left(\hat{\theta} \cdot \hat{z}^{\prime}\right) e^{j \beta\left(\hat{r} \cdot \hat{r}^{\prime}\right) z^{\prime}} d z^{\prime}=\left.\mu I_{0} \frac{e^{-j \beta r}}{4 \pi r}\left(\hat{\theta} \cdot \hat{z}^{\prime}\right) \frac{e^{j \beta\left(\hat{r} \cdot \hat{r}^{\prime}\right) z^{\prime}}}{j \beta\left(\hat{r} \cdot \hat{r}^{\prime}\right)}\right|_{-L / 2} ^{L / 2} \\
= & \mu I_{0} \frac{e^{-j \beta r}}{4 \pi r}\left(\hat{\theta} \cdot \hat{z}^{\prime}\right) \frac{2 j \sin \left(\beta\left(\hat{r} \cdot \hat{r}^{\prime}\right) \frac{L}{2}\right) L / 2}{j \beta\left(\hat{r} \cdot \hat{r}^{\prime}\right) \frac{L}{2}}=\mu_{0} L I_{0} \frac{e^{-j \beta r}}{4 \pi r}\left[\left(\hat{\theta} \cdot \hat{z}_{n}^{\prime}\right) \operatorname{sinc}\left[\frac{\beta L}{2}\left(\hat{r} \cdot \hat{r}_{n}^{\prime}\right)\right]\right],
\end{aligned}
$$

where $L$ is the length of the short dipole. Then, in the far-field region, it is seen that

$$
\bar{E}_{\theta} \simeq-j \omega A_{\theta}
$$


which produces

$$
\begin{aligned}
\bar{E}_{\theta} \simeq-j \omega \mu_{0} L I_{0} \frac{e^{-j \beta r}}{4 \pi r}\left[\left(\sin \theta_{n} \cos \theta\left(\cos \left(\phi-\phi_{n}\right)\right)\right.\right. \\
\left.\left.\quad-\sin \theta \cos \theta_{n}\right) \operatorname{sinc}\left(\frac{\beta L}{2}\left(\sin \theta_{n} \sin \theta\left(\cos \left(\phi-\phi_{n}\right)\right)+\cos \theta \cos \theta_{n}\right)\right)\right] .
\end{aligned}
$$

The normalized Field pattern is [2].

$$
\begin{aligned}
F(\theta, \phi) \simeq & {\left[\left(\sin \theta_{n} \cos \theta\left(\cos \left(\phi-\phi_{n}\right)\right)\right.\right.} \\
& \left.\left.-\sin \theta \cos \theta_{n}\right) \operatorname{sinc}\left(\frac{\beta L}{2}\left(\sin \theta_{n} \sin \theta\left(\cos \left(\phi-\phi_{n}\right)\right)+\cos \theta \cos \theta_{n}\right)\right)\right] .
\end{aligned}
$$

Equation 2.13 describes the field pattern of the dipole that is arbitrarily oriented and arbitrarily positioned.

A single dipole antenna is studied initially. The length of the short dipole is chosen as $\lambda / 50$ and the position is located at $(x, y, z)=(0,0,0)$, as shown in Figure 2.4

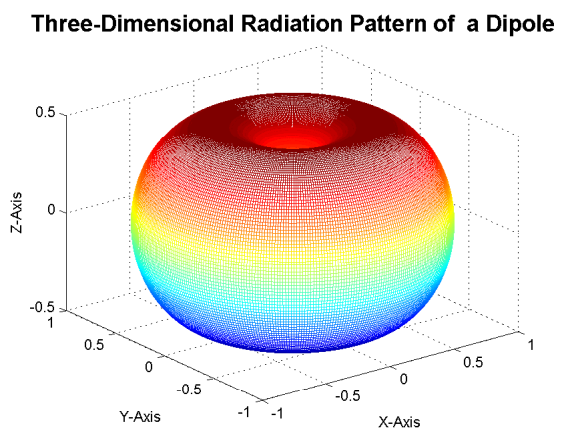

Figure 2.5: Three-dimensional radiation pattern of short dipole. 


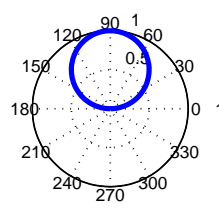

YZ Cut

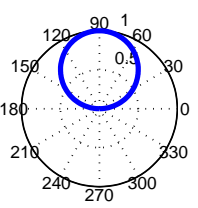

XZ Cut

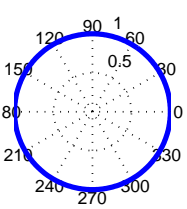

XY Cut

Figure 2.6: Cross-section of field pattern in $y z, x z$, and $x y$ planes.

The simulation results for one short dipole antenna are displayed. In Figure 2.5 the plot represents the three-dimensional radiation pattern, while Figure 2.6 represents the cross-section of the field pattern in $y z, x z$, and $x y$ planes of a dipole. The directivity is 1.4998 , which would be 1.7605 $\mathrm{dB}$, and the the half power beamwidth is 90 degree.

\subsection{Antenna Arrays}

Antenna arrays are a set of antennas arranged to provide highly directive patterns. Generally an array of $N$ dipoles, that are arbitrary,oriented and located are specified by five coordinates, three positional and two directional. First of all, as a simple application of the $1 \mathbf{D}$ antenna array formulas, it is considered that a two linear array of dipoles are positioned along the $x$-axis and oriented in the $z$-axis direction as shown in Figure 2.7, without controller system. The $y$-axis and $z$-axis have no element, meaning that there is not a dipole antenna on either axis. 


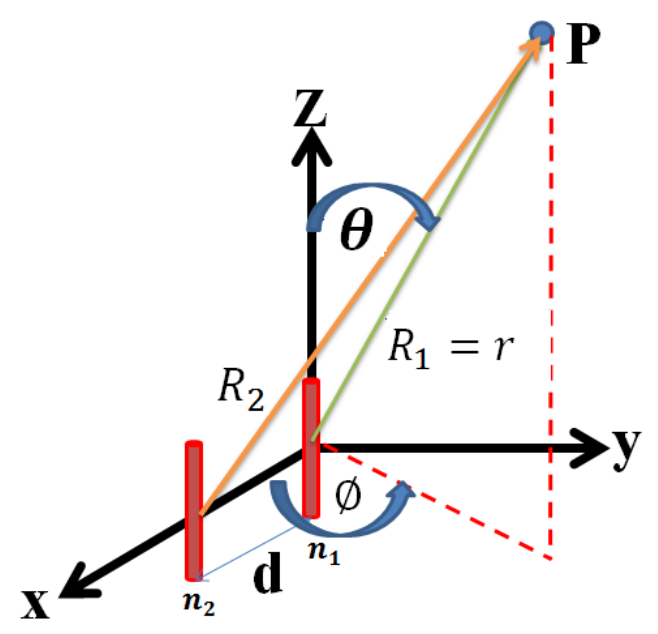

Figure 2.7: Geometry of a two-element short dipole arrays.

For one-dimensional arrays with elements along the $x$-axis the total field pattern in this case is found as:

$$
\begin{aligned}
& F(\theta, \phi) \\
& \simeq\left[\left(\sin \theta_{n} \cos \theta\left(\cos \left(\phi-\phi_{n}\right)\right)\right.\right. \\
& \left.\left.\quad-\sin \theta \cos \theta_{n}\right) \operatorname{sinc}\left(\frac{\beta L}{2}\left(\sin \theta_{n} \sin \theta\left(\cos \left(\phi-\phi_{n}\right)\right)+\cos \theta \cos \theta_{n}\right)\right)\right] e^{\left\{j \beta\left(x_{n} \sin \theta \cos \phi\right)\right\}} .
\end{aligned}
$$

The simulation results for two dipole antennas are displayed in Figure 2.8 . The right plot represents the three-dimensional radiation pattern for two dipoles. The left plot represent the cross-section of field pattern in $y z, x z, x y$ planes of the array antenna. 

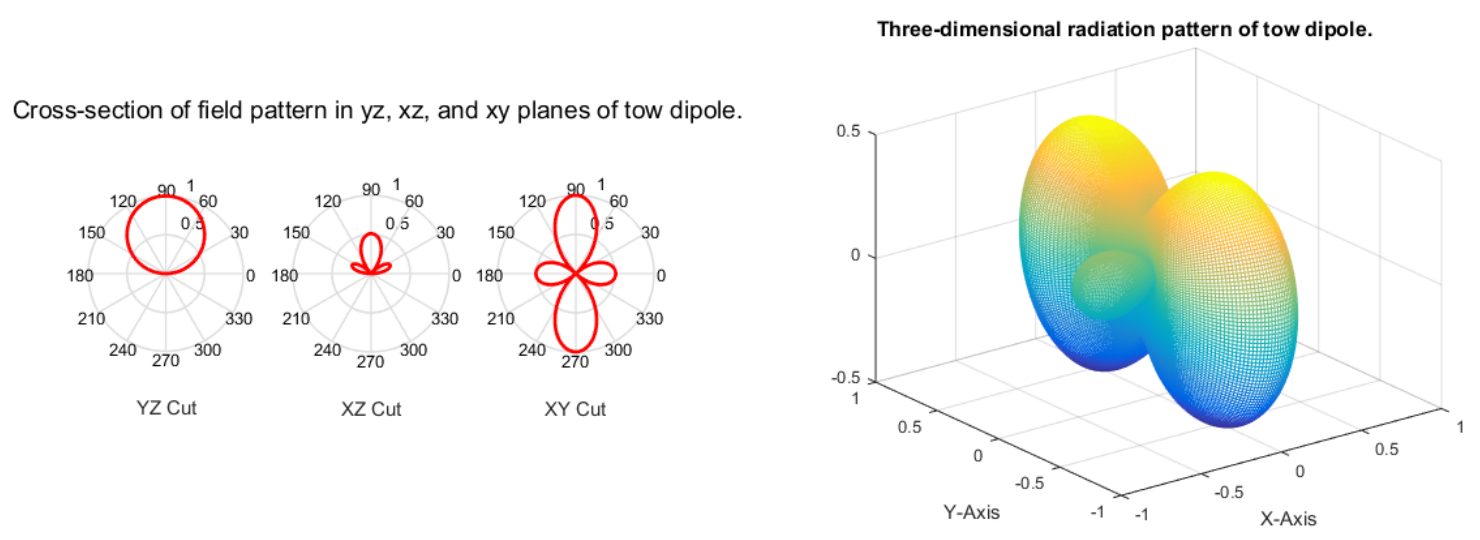

Figure 2.8: Geometry of a two-element array positioned along the $x$-axis

The directivity is defined as [2].

$$
D=\frac{4 \pi}{\Omega_{A}}
$$

where $\Omega_{A}$ is the beam solid angle defined by

$$
\Omega_{A}=\int_{0}^{2 \pi} \int_{0}^{\pi}|F(\theta, \phi)|^{2} \sin (\theta) d \theta d \phi
$$

so the directivity is

$$
D=\frac{4 \pi}{\int_{0}^{2 \pi} \int_{0}^{\pi}|F(\theta, \phi)|^{2} \sin (\theta) d \theta d \phi} .
$$

Numerical computation of equation (2.17), uses Matlab code to find the value of array element spacing, $d$, that gives the maximum directivity as shown in Figure 2.9. This figure shows the relation between $d$ and the directivity of the array. The directivity maximum is $D^{*}=4.5$ when the distance between two dipole $d^{*}=0.67$ meter where $d^{*}$ is the optimal position that gives the maximum directivity. The frequency of excetation is $3 \mathrm{MHz}$. 


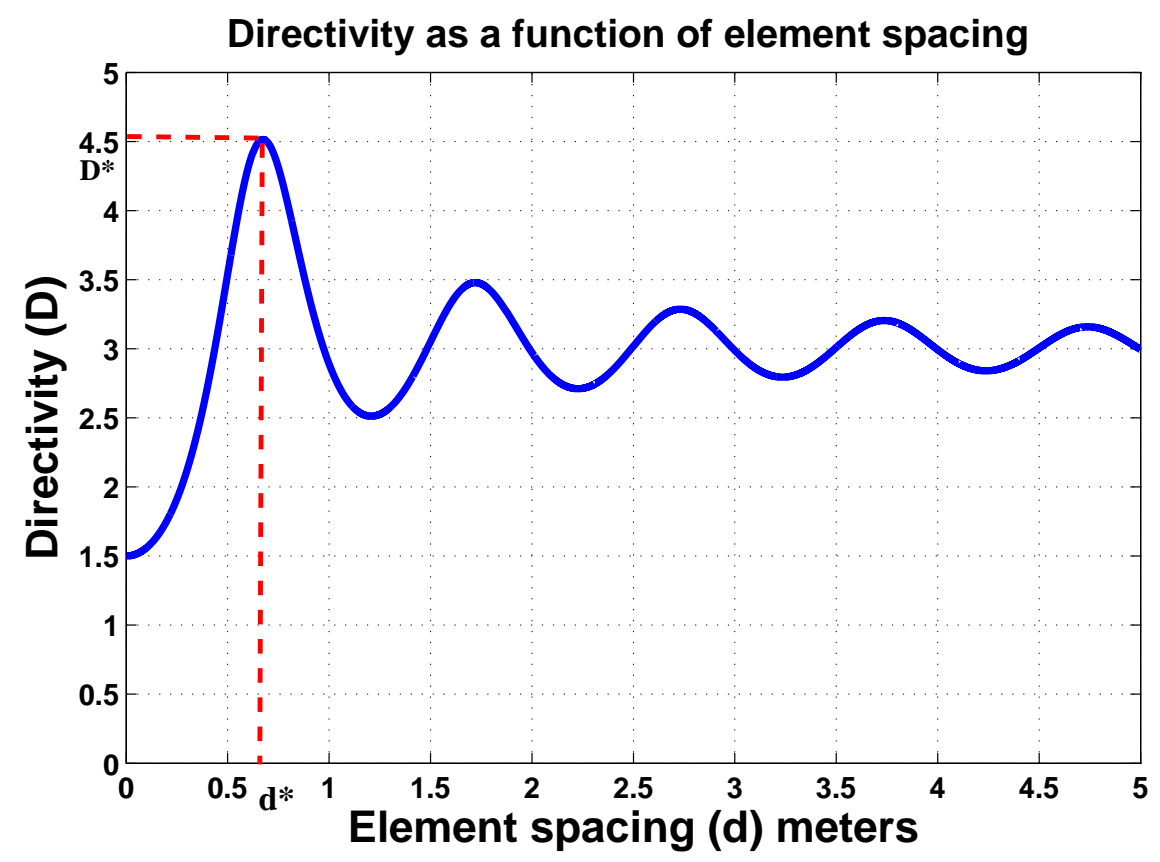

Figure 2.9: Directivity versus element spacing.

\subsection{Kinematic Point-Mass Model Simulations}

Dealing with the general nonlinear system

$$
\begin{gathered}
\dot{x}=f(x, u), \\
y=J(x),
\end{gathered}
$$

where $x \in \mathbb{R}^{\mathrm{n}}$, is the state, $u \in \mathbb{R}$ is the input, $y \in \mathbb{R}$ is the performance output, and the functions $f: \mathbf{D} \times \mathbb{R} \rightarrow \mathbb{R}^{\mathrm{n}}$ and $J: \mathbf{D} \rightarrow \mathbb{R}^{\mathrm{n}}$ are sufficiently smooth on $\mathbf{D} \subseteq \mathbb{R}^{\mathrm{n}}$. For the simplification of analysis, we assume $\mathbf{D}=\mathbb{R}^{\mathrm{n}}$. The ESC problem then can be stated as

$$
\max _{x \in n} \quad J(x) \quad \text { subject to } \quad \dot{x}=f(x, u) .
$$

For simulation purposes, we consider a one-dimensional case where $n=1$ and two vehicles are used the first one is fixed at the origin $(0,0,0)$, the second, moves on the $x$-axis and both have 
antennas that are fixed. The dynamics of the vehicle $x$ satisfy whose position is

$$
\begin{aligned}
& \dot{x}^{i}=u^{i}, \\
& y=J\left(x_{1}, u\right)=\text { Directivity, } \\
& u=\arg \max _{0<x_{1}<l} J\left(x_{1}, u\right),
\end{aligned}
$$

where $x_{1}$ is the position of the vehicle and $u$ is the control input for the autonomous vehicle. We consider the simplest case of a velocity-actuated point mass. The block diagram shows the vehicles in $1 \mathbf{D}$ and the fixed orientation of the antenna shown in Figure 2.10.

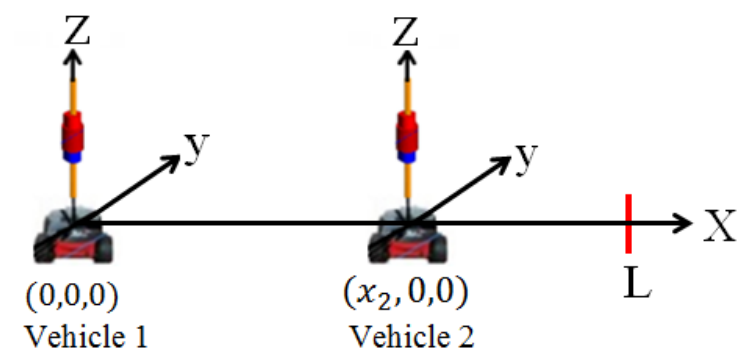

Figure 2.10: Two vehicles in 1D and fixed orientation of antenna.

The objective output $y$ represents the directivity, while the optimal value $x^{*}$ is the best position $x$ where the best $y$ is obtained. 


\section{CHAPTER III}

\section{D FIXED ANTENNA ORIENTATION}

\subsection{Two Vehicles in $1 D$ with Fixed Antenna Orientation at $\left(\theta_{1,2}, \phi_{1,2}\right)=(0,0)$}

There are two autonomous vehicles carrying directional antennas to find maximum directivity by changing the position of the vehicles along the $x$-axis. Vehicle 1 is fixed at the origin position, with the fixed antenna orientation being $\left(\theta_{1}, \phi_{1}\right)=(0,0)$, and Vehicle 2, with the fixed antenna orientation being $\left(\theta_{2}, \phi_{2}\right)=(0,0)$, moves in the direction of the $x$-axis, while having a controller looking for the best position that gives the optimal directivity, as shown in Figure 2.10 for simplicity. PESC is implemented in the system, and NOESC is implemented using both DS and PSO.

\subsubsection{Perturbation-based Extremum Seeking Control}

MATLAB was used to implement the PESC algorithm. The parameters of the PESC loop are chosen as loop perturbation frequency $w_{x}=20$ units (rad/s), loop perturbation amplitude $A_{x}=$ 0.01, loop gain $C_{x}=3$, and the filter $h=1$. The start position of the vehicle is $\left(x_{2}(0)\right)=0$. Unknown signal $J$ is shown in Figure 3.1. It illustrates the relationship between time and the objective function $J$. Figure 3.1 shows that the PESC is indeed able to find the optimum directivity $J^{*}$ even though the input varies with time. As expected, $J$ converges to a periodic motion around $J^{*}$. In addition, it shows that the objective function was driven toward its optimum rapidly and kept at the optimum by the method. The noise that appears before the objective function reaches its 
optimum value of 4.8592 is caused by the fact that the PESC method uses a sinusoidal perturbation signal.

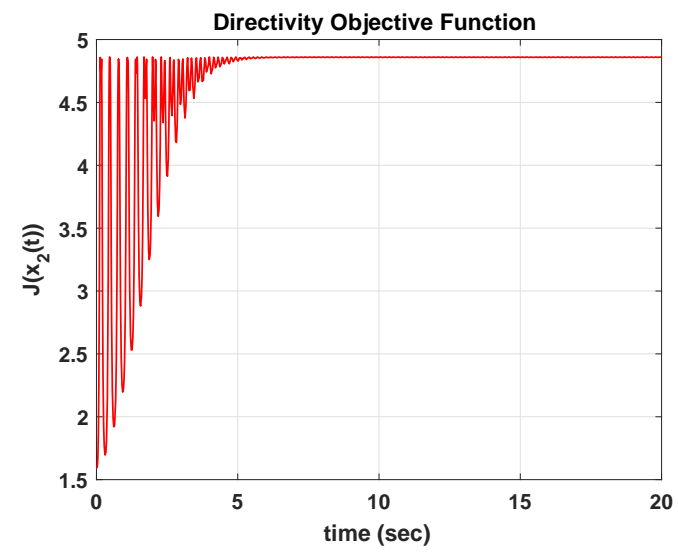

Figure 3.1: Objective function for PESC in a 1D case (2 vehicles).

Next, by observing the relationship between time and position, shown in Figure 3.2, it is clear that the PESC causes the optimum position to converge to a neighborhood of $x^{*}$. This optimum position of $x^{*}(0.6593)$, which is the average of the sine wave, is the point where the $J$ function reaches the value of $J^{*}$. In this figure it is evident that the oscillation is caused by the effect of the sine wave used in the PESC. 


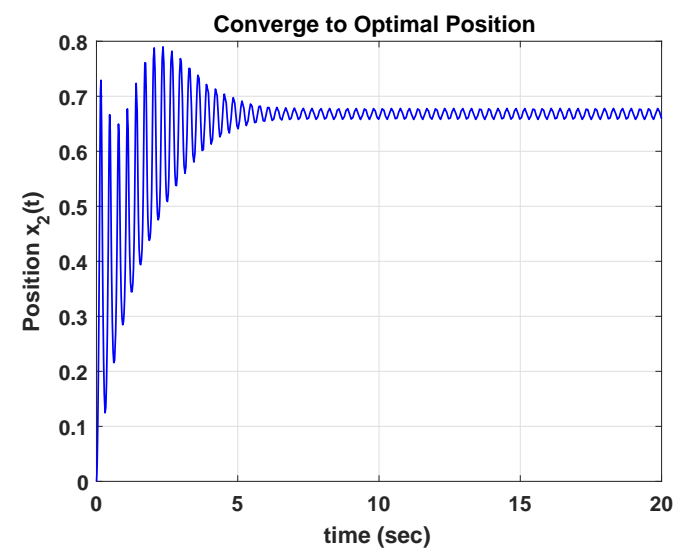

Figure 3.2: Position for PESC in a 1D case (2 vehicles).

The relationship between time and the controller can be seen in Figure 3.3. Here it is shown that around the optimum values of the objective function and the position, the controller converges to a neighborhood of zero.

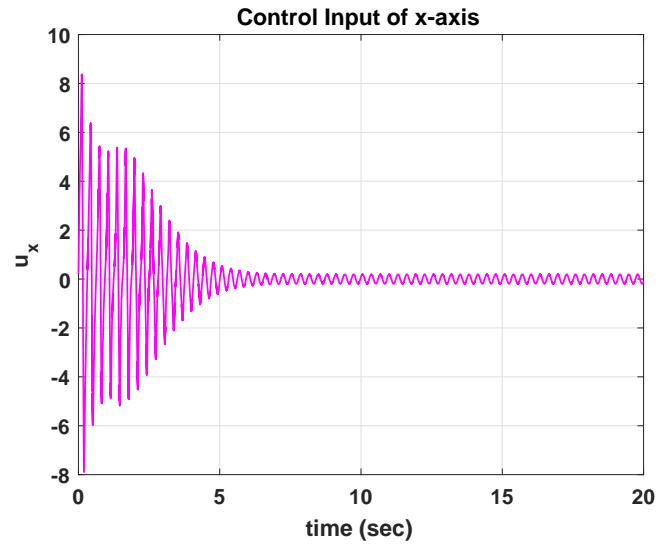

Figure 3.3: Control input of $x$-axis for PESC in a 1D case (2 vehicles). 


\subsubsection{Numerical Optimization-based Extremum Seeking Control Using DS}

The same problem is now solved using the NOESC method with direct search. The results are shown in Figures 3.4, 3.5, and 3.6. From Figure 3.4 it can be seen how the objective function changes with time. Again the objective function converges to the value 4.850, which represents the optimum value of the objective function. The convergence here happens very smoothly as shown in the figure.

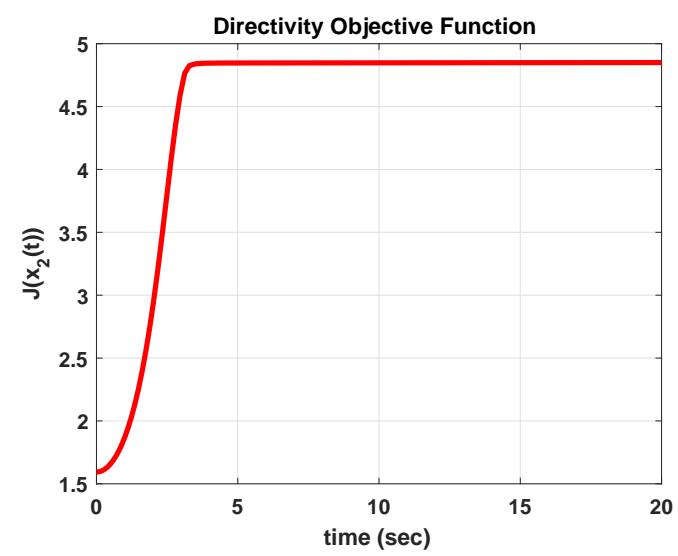

Figure 3.4: Objective function for NOESC-DS in a 1D case (2 vehicles).

Figure 3.5 illustrates the position $x_{2}$ as a function of time. As shown in this figure, the optimum position obtained by using the direct search algorithm [54] is 0.6519 . The convergence for the position towards its optimum value also comes in a smooth curve. 


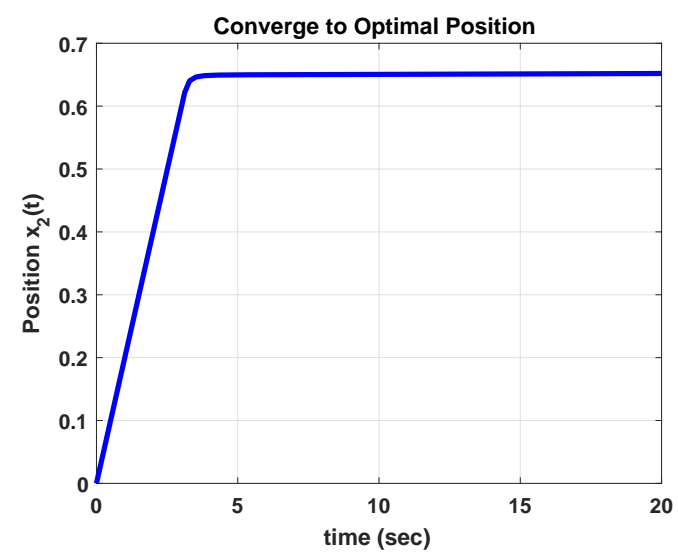

Figure 3.5: Position for NOESC-DS in a 1D case (2 vehicles).

The relationship between time and the controller can be seen in Figure 3.6. Here it is shown that around the optimum values of the objective function and the position, the controller converges to a neighborhood of zero.

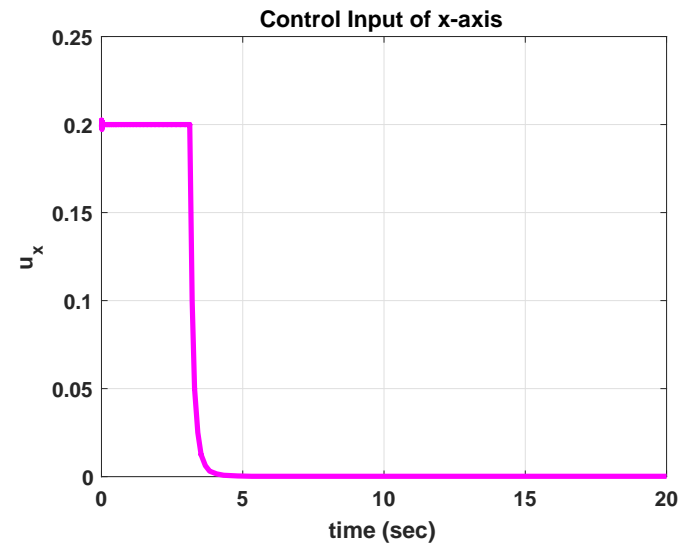

Figure 3.6: Control input of $x$-axis for NOESC-DS in a 1D case (2 vehicles). 


\subsubsection{Numerical Optimization-based Extremum Seeking Control Using PSO}

The same problem is now solved using the NOESC method with PSO. Splines interpolation is a featured function in MATLAB. PSO algorithm is run on the splines interpolation $\hat{J}$, an approximation of $J$ that gets better as the vehicle collects more points, which produces the next regulation point to go to from $\hat{J}$. Only when using PSO, $\hat{J}$ is considered the objective function. The results are shown in Figures 3.7, 3.8, and 3.9. From Figure 3.7 it can be seen how the objective function changes with time. Again the objective function converges to the value 4.8614, which represents the optimum value of the objective function. The convergence here happens very smoothly as shown in the figures.

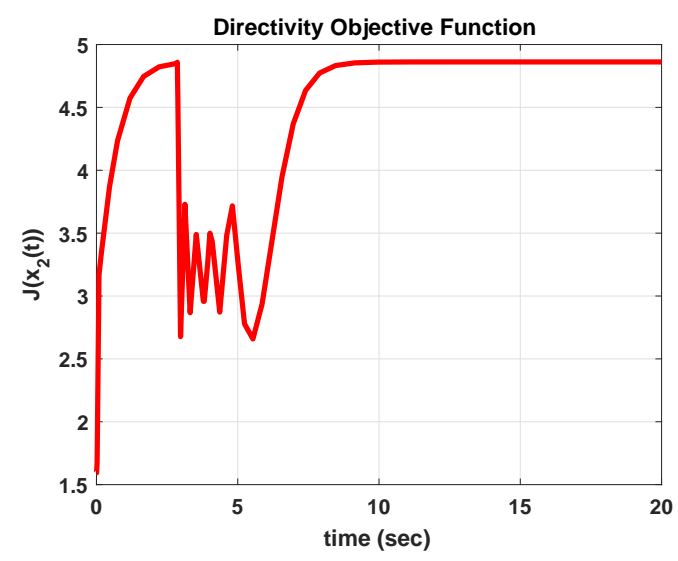

Figure 3.7: Objective function for NOESC-PSO in a 1D case (2 vehicles).

Figure 3.8 illustrates the position $x_{2}$ as a function of time. As shown in this figure, the optimum position obtained by using the PSO algorithm [65] is 0.6679 . The convergence for the position towards its optimum value also comes in a smooth curve. 


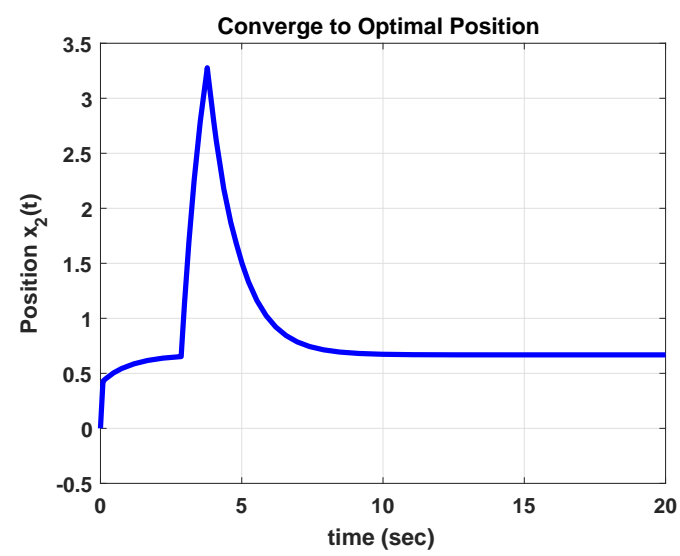

Figure 3.8: Position for NOESC-PSO in a 1D case (2 vehicles).

The relationship between time and the controller can be seen in Figure 3.9. Here it is shown that around the optimum values of the objective function and the position, the controller converges to a neighborhood of zero. The convergence happens at about five seconds.

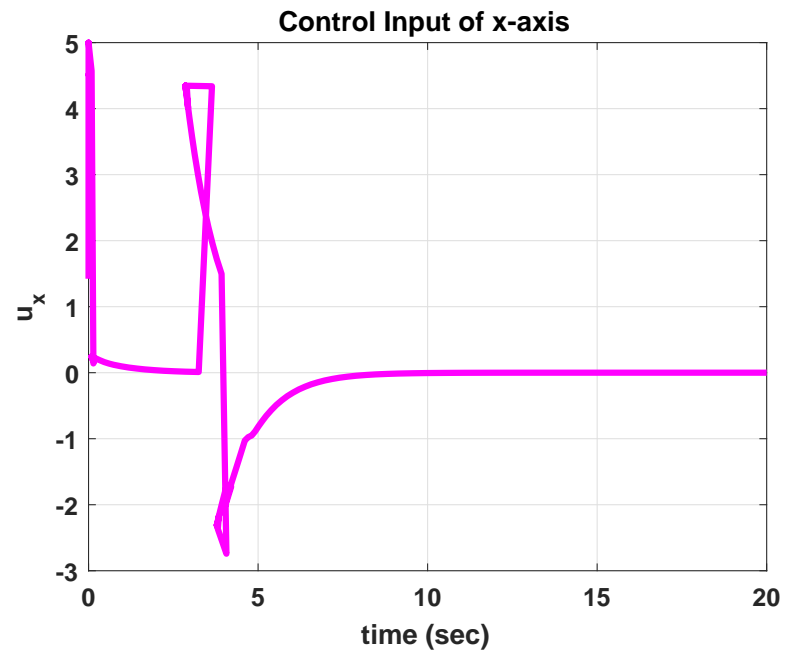

Figure 3.9: Control input of $x$-axis for NOESC-PSO in a 1D case (2 vehicles). 


\subsubsection{Comparison Results of PESC and NOESC with DS and PSO}

In comparing the PESC, NOESC with Direct Search, and NOESC with PSO, which pairs Figure 3.1 with Figure 3.4 with Figure 3.7. Figure 3.2 with Figure 3.5 with Figure 3.8, and Figure 3.3 with Figure 3.6 with Figure 3.9 , it can be noticed that each pair of figures performs the same task. Each pair achieves the same final result, despite the fact that different algorithms were used to obtain those results. One major difference though, is the effect of the sine wave that can be clearly seen when using the PESC method. On the other hand, when the NOESC with Direct Search method is used, the convergence process happens in a smooth matter. NOESC with PSO is not smooth because it is an approximation of $J$. In addition, the NOESC with DS method has a faster convergence of 4 second than the PESC and NOES with PSO method, which have a convergence of 5 second and 9 second respectively. Another major difference is that Figure 3.3, which is the control input of the PESC, has a control energy of 4.6402, the NOESC with Direct Search has a control input of 0.0301 shown in Figure 3.6, and the NOESC with PSO, control input shown in Figure 3.9, has a control energy of 9.1528. As seen, the control energy with the NOESC with Direct Search is lower than the control energy with PESC and NOESC with PSO, making it the best result in comparing the controller energy. Finally, comparing the PESC, NOESC with Direct Search, and NOESC with PSO, the NOESC-DS had the best results overall, which can be seen in Table 3.1. Equation 3.1 shows how to compute the control energy, i.e, its mean square value

$$
\text { ControlEnergy }=\frac{1}{T} \int_{0}^{T} u^{2}(t) d t
$$


Table 3.1: Comaparison results of three algorithms for Case 2 Vehicles

\begin{tabular}{|l|c|c|c|}
\hline ESC & Optimum objective & Control energy & Optimum position \\
\hline PESC & 4.8592 & 4.6402 & $x^{*}=0.6593$ \\
\hline NOESC-DS & 4.850 & 0.0301 & $x^{*}=0.6519$ \\
\hline NOESC-PSO & 4.8614 & 9.1528 & $x^{*}=0.6679$ \\
\hline
\end{tabular}

\subsection{Three Vehicles in 1D with Fixed Antenna Orientation}

Unlike the first case, in this part of the 1D study, three vehicles, one fixed and two moving, were analyzed. One is moving along the negative $x$-axis, and the other moves along the positive $x$-axis. Each one of these vehicles is equipped with a vertically orientated dipole antenna. While these antennas are fixed in this case, every vehicle has one degree of freedom. The one degree, which is for the position of the vehicles, are $\left(x_{1}, 0,0\right)$ and $\left(x_{2}, 0,0\right)$ as shown in the Figure 3.10 . The system should be able to test every possible value for the two states, $x_{1}$ and $x_{2}$, within the range $[-L, L]$. The optimal value for the position gives the optimal objective function which maximizes the directivity.

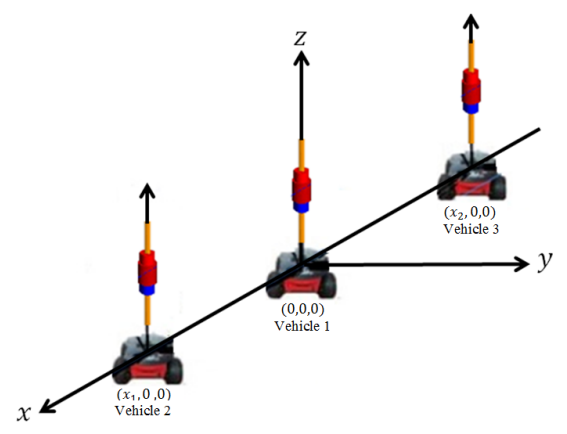

Figure 3.10: Three vehicles in 1D and fixed orientation antenna. 


\subsubsection{Perturbation-based Extremum Seeking Control}

MATLAB is used to implement the PESC algorithms. The parameters of the ESC loop are chosen as loop perturbation frequency, $w_{x_{1,2}}=20 \mathrm{rad} / \mathrm{s}$, loop perturbation amplitude $A_{x_{1,2}}=0.04$, loop gain $C_{x_{1,2}}=1$, the filter $h=1$, the state $x_{1}$ being between the range of $[-10,0]$, and the state $x_{2}$ being in the range of $[0,10]$. The initial condition of the vehicles is $\left.x_{1}(0)=-0.2, x_{2}(0)\right)=0.2$. All the initial conditions are in meters. The signal $J$ is shown in Figure 3.11. The optimal objective function value is 8.2477 , which is an average of a disturbance wave.

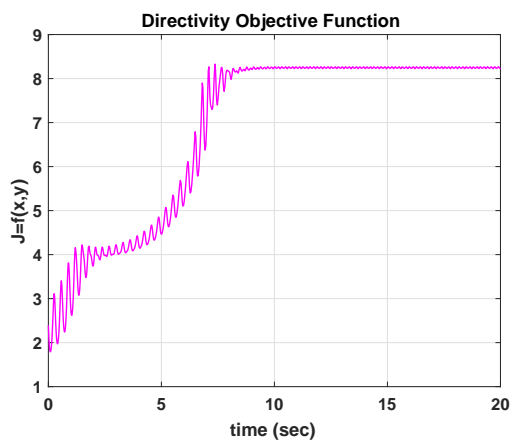

Figure 3.11: Objective function for PESC in a 1D case (3 vehicles).

In Figure 3.12, the vehicles' position shows that the vehicles have moved from the initial condition, which is $(-0.2,0.2)$, to the optimal position, which is $x_{1}=-0.7933$ and $x_{2}=0.7808$, and both of these numbers are an average of the pertubation signal. 


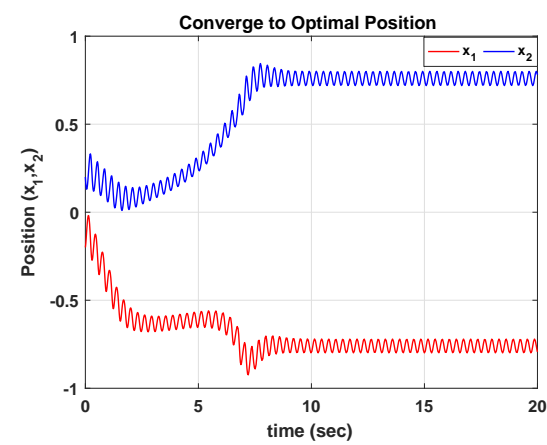

Figure 3.12: Position for PESC in a 1D case (3 vehicles).

When the optimal objective function and the optimal position of $x_{1}$ and $x_{2}$ are found, the controller converges to an average of neighborhood of zero. This is presented in Figure 3.13 .

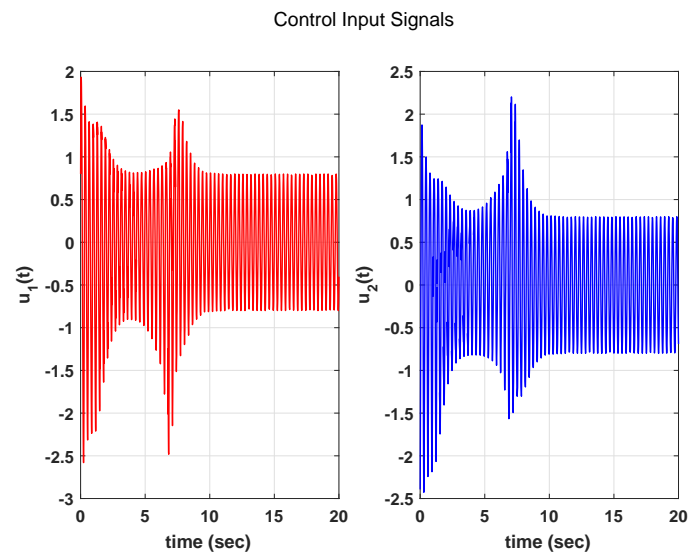

Figure 3.13: Control input of $x$-axis for PESC in a 1D case (3 vehicles).

\subsubsection{Numerical Optimization-based Extremum Seeking Control Using DS}

Now implementing the NOESC with Direct Search method instead of the PESC method, the results are shown in Figure 3.14, Figure 3.15, and Figure 3.16. From Figure 3.14 it can be seen how the objective function changes with time. Notice that the objective function converges to the 
value 8.3343 , which represents the optimum value of objective function, again. The convergence here happens very fluently as shown in the Figure 3.14. Exhibited in Figure 3.15 is the relationship

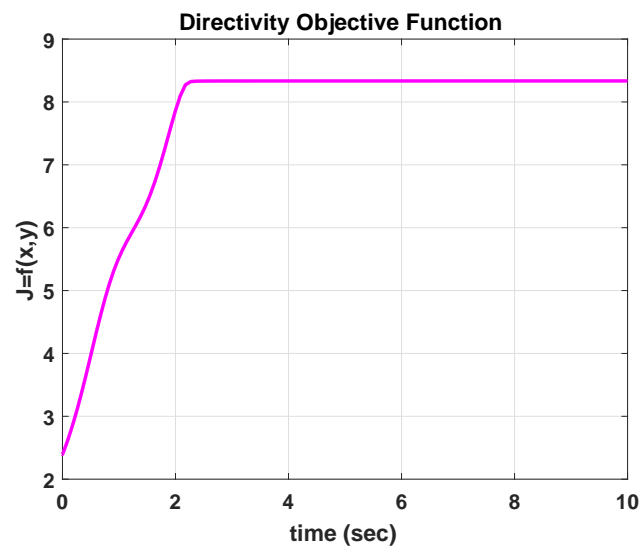

Figure 3.14: Objective function for NOESC-DS for 1D case (3 vehicles).

between the time and position. This shows that the optimal value is $x_{1}=-0.7580$ and $x_{2}=0.7579$. These results give the optimal objective value.

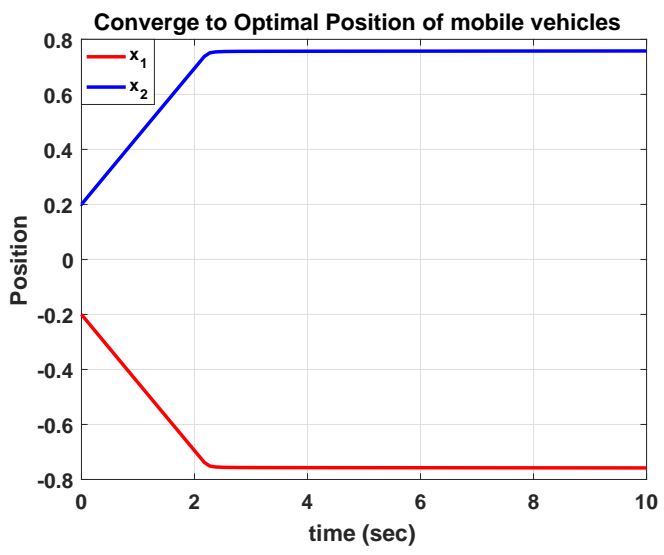

Figure 3.15: Position for NOESC-DS in a 1D case (3 vehicles). 
The controller input of $x_{1}$ is $u_{1}$, and the controller input of $x_{2}$ is $u_{2}$. These two controllers are able to achieve the optimal position. And the optimal position found gives the optimal objective directivity. It converges to a neighborhood of zero, which is presented in Figure 3.16 .

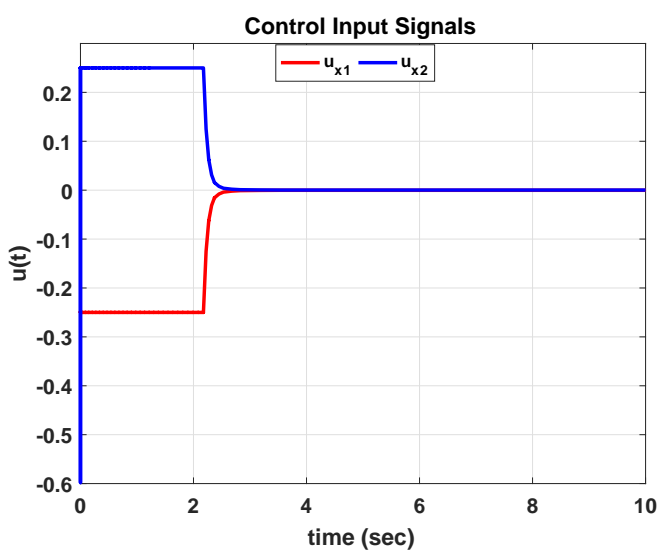

Figure 3.16: Control input of $x$-axis for NOESC-DS in a 1D case (3 vehicles).

\subsubsection{Numerical Optimization-based Extremum Seeking Control Using PSO}

On MATLAB, the TriScatteredInterp function is used because it performs interpolation on a scattered dataset that resides in $2 \mathbf{D}$ or $3 \mathbf{D}$ space, in this case $\left(x_{1}, x_{2}\right)$, which produces $\hat{J}$. Only when using PSO, $\hat{J}$ is considered the objective function. Implementing the NOESC with PSO method instead of the PESC method, while maintaining the same initial condition and state ranges, yielded the results shown in Figures 3.30, 3.31, and 3.19. From Figure 3.30 it can be seen how the objective function changes with time. Notice that the objective function converges to the value 8.3315 , which represents the optimum value of objective function. The convergence here happens very fluently as shown in the Figure 3.30 . 


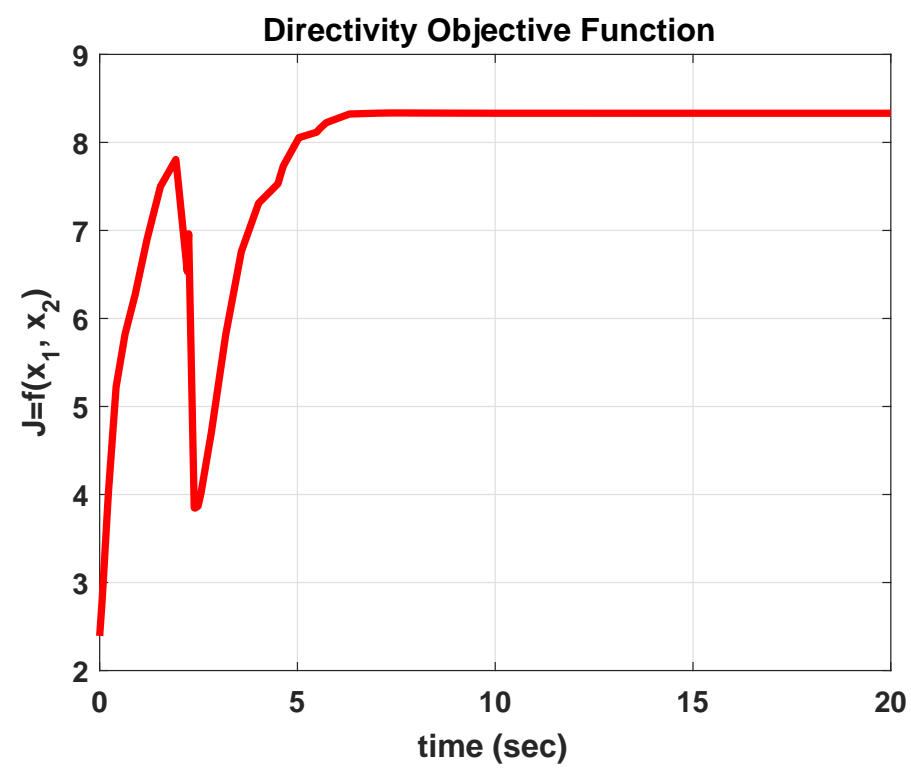

Figure 3.17: Objective function for NOESC-PSO in a 1D case (3 vehicles).

Figure 3.31 shows the relationship between the time and position. This shows that the optimal value is $x_{1}=-0.7673$ and $x_{2}=0.7548$. These results give the optimal objective value.

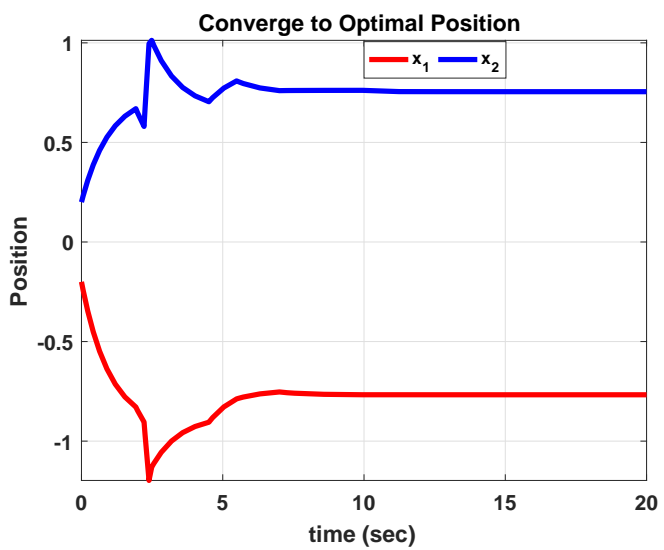

Figure 3.18: Position for NOESC-PSO in a 1D case (3 vehicles). 
The controller input of $x_{1}$ is $u_{1}$, and the controller input of $x_{2}$ is $u_{2}$. These two controllers are able to achieve the optimal position, which provides the optimal objective directivity. It converges to a neighborhood of zero, which is presented in Figure 3.19 .

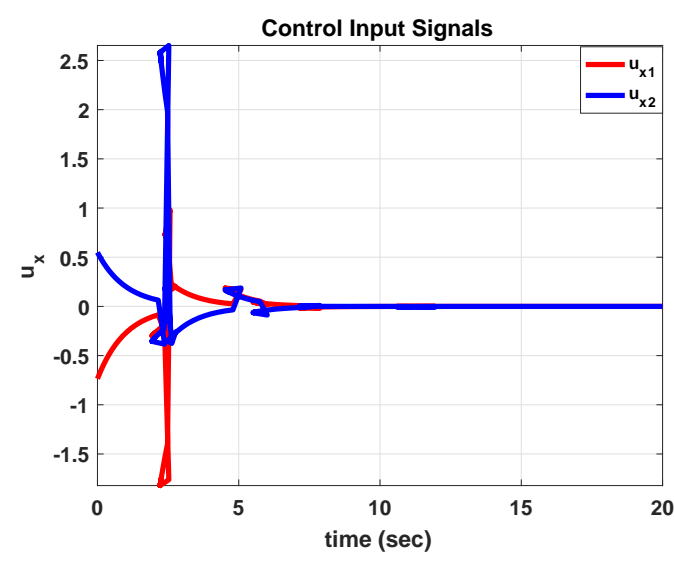

Figure 3.19: Control input of $x$-axis for NOESC-PSO in a 1D case (3 vehicles).

\subsubsection{Comparison Results of PESC and NOESC with DS and PSO}

The three algorithms, PESC, NOESC with Direct Search, and NOESC with PSO, though each is different, obtained similar results, but NOESC-DS is better because it has the lowest control energy. The results can be seen in Table 3.2 .

Table 3.2: Comparison results of three algorithms for Case 3 Vehicles

\begin{tabular}{|l|l|l|l|}
\hline ESC & Optimum Objective & Control energy & Optimum position \\
\hline \multirow{2}{*}{ PESC } & 8.2477 & $U_{1}=0.6775$ & $x_{1}=-0.7933$ \\
& & $U_{2}=0.6926$ & $x_{2}=0.7808$ \\
\hline \multirow{2}{*}{ NOESC-DS } & \multirow{2}{*}{ N.3343 } & $U_{1}=0.0471$ & $x_{1}=-0.7580$ \\
& & $U_{2}=0.0484$ & $x_{2}=0.7579$ \\
\hline \multirow{2}{*}{ NOESC-PSO } & 8.3315 & $U_{1}=0.4576$ & $x_{1}=-0.7673$ \\
& & $U_{2}=0.7749$ & $x_{2}=0.7548$ \\
\hline
\end{tabular}




\subsection{Sensitivity of Extremum Seeking Control to Changes the Initial Conditions in Antenna Array Applications}

In this section, we will compare two algorithms, Perturbation-based Extremum Seeking Control (PESC) and Numerical Optimization based Extremum Seeking Control using Particle Swarm Optimization (NOESC-PSO). The sensitivity of the two methods to initial condition is examined via simulations.

\subsubsection{Perturbation-based Extremum Seeking Control at the Initial Condition $( \pm 0.5)$}

MATLAB is used to implement the PESC algorithms. The parameters of the ESC loop are chosen as loop perturbation frequency, $w_{x_{1,2}}=50 \mathrm{rad} / \mathrm{s}$, loop perturbation amplitude $A_{x_{1,2}}=0.02$, loop gain $C_{x_{1,2}}=1$, the filter $h=1$, the state $x_{1}$ being between the range of $[0,7]$, and the state $x_{2}$ being in the range of $[-7,0]$. The initial condition of the vehicles are $\left.x_{1}(0)=0.5, x_{2}(0)\right)=$ -0.5 . The signal $J$ is shown in Figure 3.20 . The optimal objective function value converges to the maximum global optimal objective function of 8.3125 , which is an average of a disturbance wave.

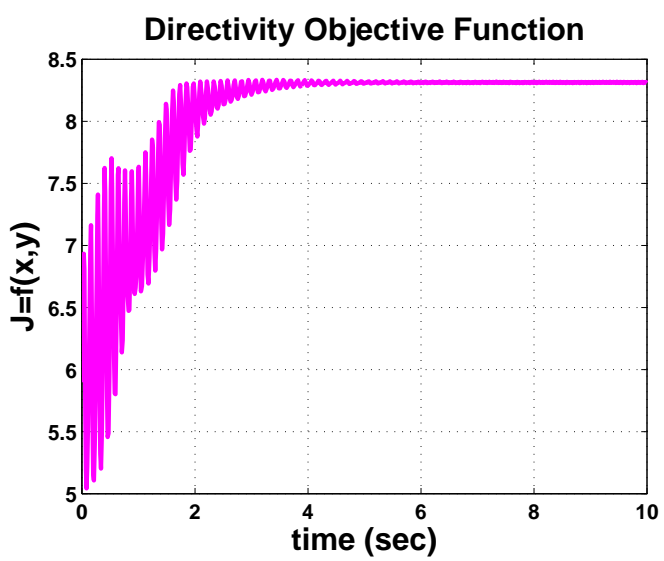

Figure 3.20: Objective function for PESC for 1D case (3 vehicles). 
In Figure 3.21, the vehicles' position shows that the vehicles have moved from the initial condition, which is $(0.5,-0.5)$, to the optimal position, which is $x_{1}=0.7505$ and $x_{2}=-0.7423$, and both of these numbers are an average of the perturbation signal.

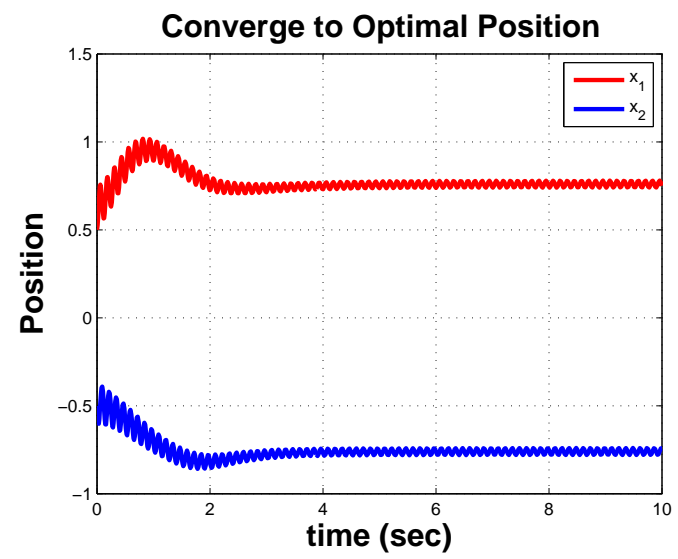

Figure 3.21: Position for PESCl for 1D case (3 vehicles).

\subsubsection{Numerical Optimization-based Extremum Seeking Control Using PSO at the Initial Condition $( \pm 0.5)$}

Using the same the TriScatteredInterp function, the NOESC with PSO method was implemented instead of the PESC method, while maintaining the same initial condition and state ranges, yielded the results shown in Figures 3.22, and 3.23. From Figure 3.22 it can be seen how the objective function changes fluently with time. Notice that the objective function converges to the value 8.3334 , which represents the optimum value of the objective function. 


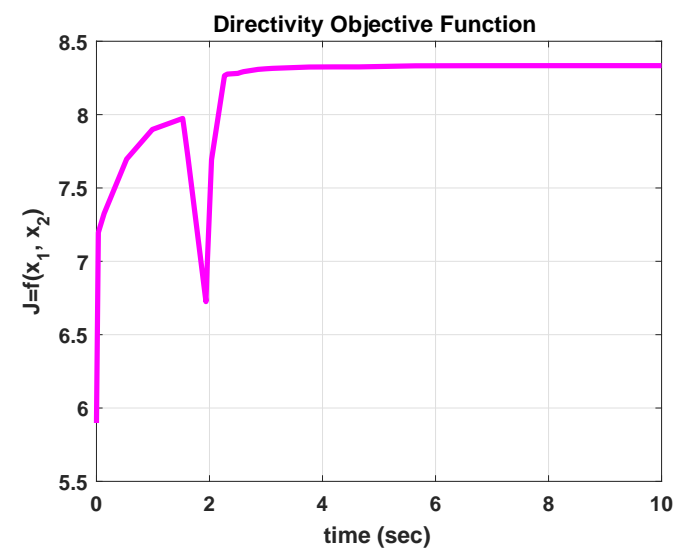

Figure 3.22: Objective function for NOESC-PSO in a 1D case (3 vehicles).

Figure 3.23 shows the relationship between the time and position. This shows that the optimal value is $x_{1}=0.7626$ and $x_{2}=-0.7556$. These results give the optimal objective value.

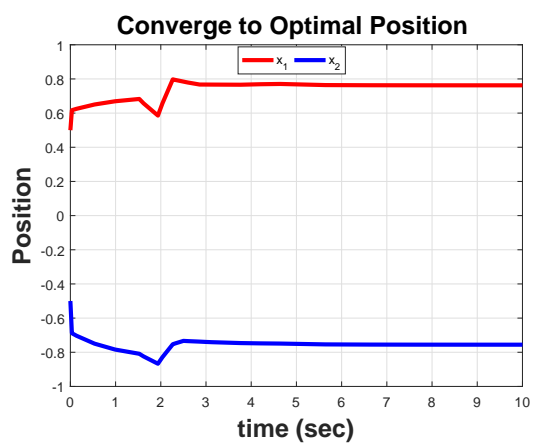

Figure 3.23: Position for NOESC-PSO in a 1D case (3 vehicles).

\subsubsection{Perturbation-based Extremum Seeking Control at the Initial Condition $( \pm 7)$}

MATLAB is used to implement the PESC algorithms. The parameters of the ESC loop are chosen as loop perturbation frequency, $w_{x_{1,2}}=50 \mathrm{rad} / \mathrm{s}$, loop perturbation amplitude $A_{x_{1,2}}=0.05$, loop gain $C_{x_{1,2}}=1$, the filter $h=1$, the state $x_{1}$ being between the range of $[0,7]$, and the state 
$x_{2}$ being in the range of $[-7,0]$. The initial condition of the vehicles is $\left.x_{1}(0)=7, x_{2}(0)\right)=-7$. The signal $J$ is shown in Figure 3.24. The optimal objective function value diverges to the local maximum objective function of 5.054, which is an average of a disturbance wave.

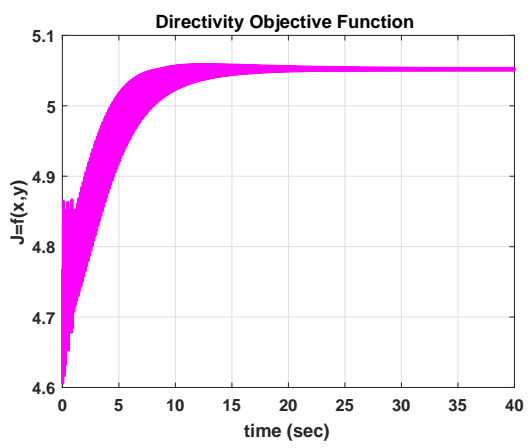

Figure 3.24: Objective function for PESC in a 1D case (3 vehicles).

In Figure 3.25, the vehicles' position shows that the vehicles have moved from the initial condition, which is $(7,-7)$, to the local position, which is $x_{1}=6.848$ and $x_{2}=-6.782$, and both of these numbers are an average of the perturbation signal.

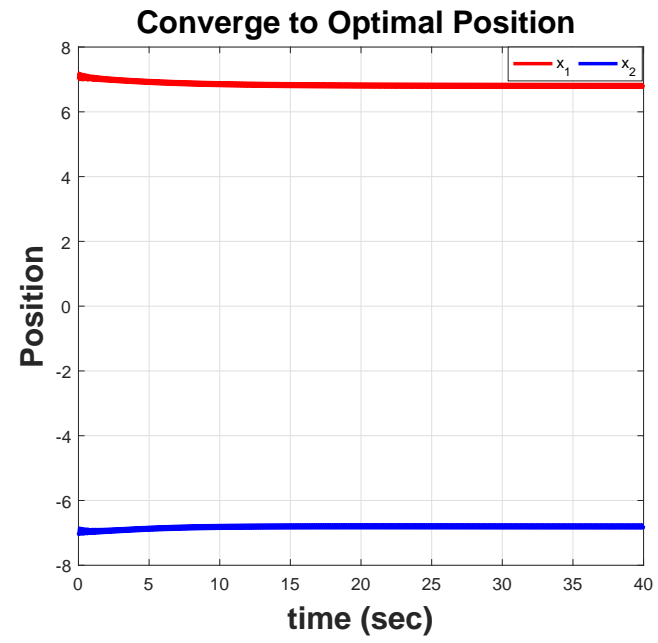

Figure 3.25: Position for PESC in a 1D case (3 vehicles). 


\subsubsection{Numerical Optimization-based Extremum Seeking Control Using PSO at the Initial Condition $( \pm 7)$}

Using the same the TriScatteredInterp function, the NOESC with PSO method was implemented instead of the PESC method, while maintaining the same initial condition and state ranges as the previous section, yielded the results shown in Figures 3.26, and 3.27. From Figure 3.26 it can be seen how the objective function changes with time. Notice that the objective function converges to the value 5.0601, which represents the global maximum objective function. The convergence here happens very fluently as shown in Figure 3.26 .

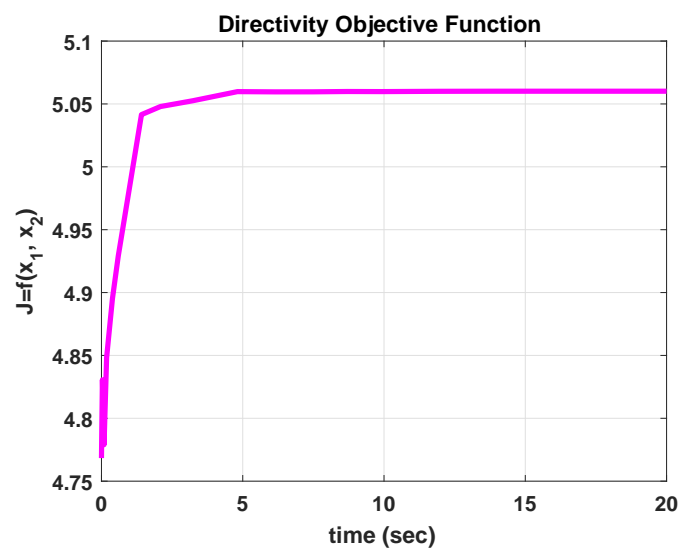

Figure 3.26: Objective function for NOESC-PSO in a 1D case (3 vehicles).

Figure 3.27 shows the relationship between the time and position. This shows that the optimal value is $x_{1}=6.8061$ and $x_{2}=-6.7970$. These results give the optimal objective value. 


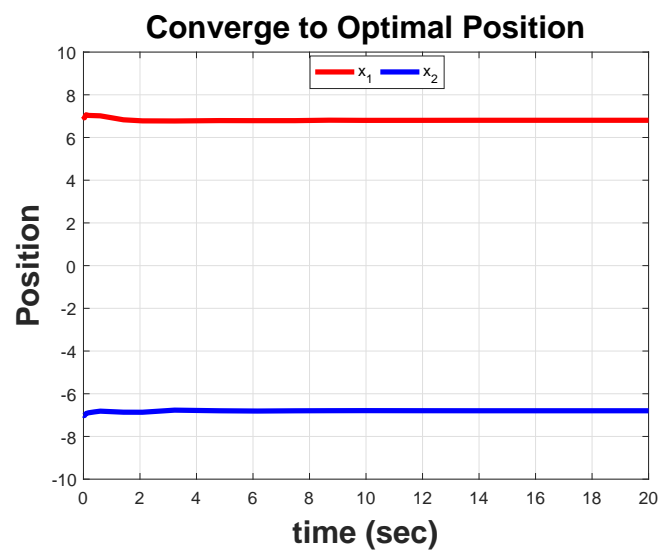

Figure 3.27: Position for NOESC-PSO in a 1D case (3 vehicles).

\subsubsection{Perturbation-based Extremum Seeking Control With a different of Initial Con- ditions}

MATLAB is used to implement the PESC algorithms. The parameters of the ESC loop are chosen as loop perturbation frequency, $w_{x_{1,2}}=50 \mathrm{rad} / \mathrm{s}$, loop perturbation amplitude $A_{x_{1,2}}=0.02$, loop gain $C_{x_{1,2}}=1$, the filter $h=1$, the state $x_{1}$ being between the range of $[0,3]$, and the state $x_{2}$ being in the range of $[-3,0]$. The initial condition of the vehicles are $x_{1}(0)=[0.5,1,1.5,2,2.5,3]$, $\left.x_{2}(0)\right)=[-0.5,-1,-1.5,-2,-2.5,-3]$. The optimum value of the objective function depends on the initial conditions, as is evident from the simulation in Figure 3.28. The loop may either converge to the optimum or diverge depending on the initial conditions. The signal $J_{i}$ is the optimal objective function where $i=1,2,3,4,5,6$. 


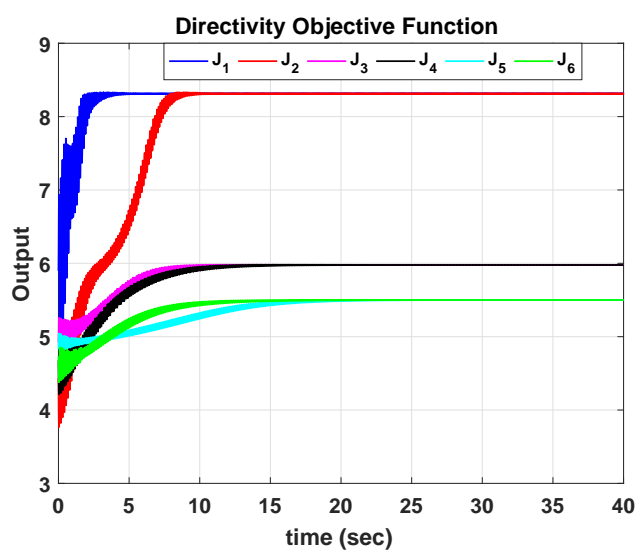

Figure 3.28: Set of objective function for PESC in a 1D case (3 vehicles).

In Figure 3.29, the vehicles' position shows that for each initial condition there is an optimal objective function. The highest objective function is the initial condition's convergence to the global maximum objective of 8.307. As for the others, two converge to 5.979 and the other two converge to 5.501. These two sets of results are the local maximum objective.

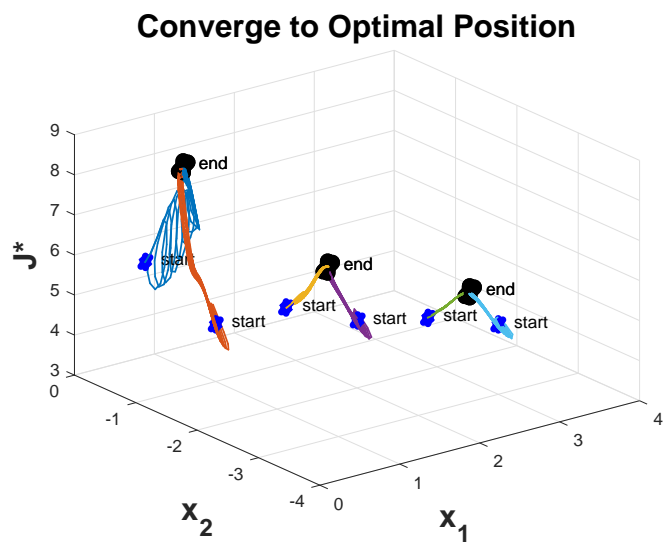

Figure 3.29: Set of position for PESC in a 1D case (3 vehicles). 


\subsubsection{Numerical Optimization-based Extremum Seeking Control Using PSO With a different of Initial Conditions}

Using the same the TriScatteredInterp function, the NOESC with PSO method was implemented instead of the PESC method, while maintaining the same initial condition and state ranges as the previous section, yielded the results shown in Figures 3.30 and 3.31 From Figure 3.30 it can be seen how the objective function changes with time. The highest objective function is the initial condition's convergence to the global maximum objective of 8.326. As for the others, two converge to the local maximum objective 5.983 and the other two converge to the local maximum objective 5.503.

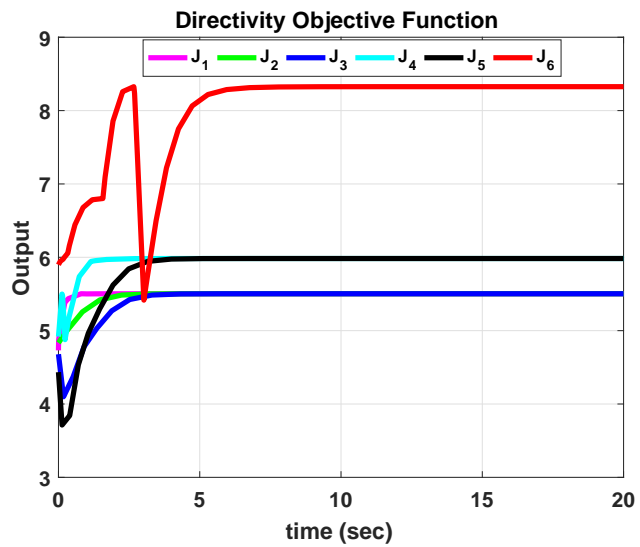

Figure 3.30: Set of objective function for NOESC-PSO in a 1D case (3 vehicles).

Figure 3.31 shows that all the differing initial conditions (represented in the graph as the word start) converge to the different maximum optimal object function (represented in the graph as the word end). 


\section{Converge to Optimal Position}

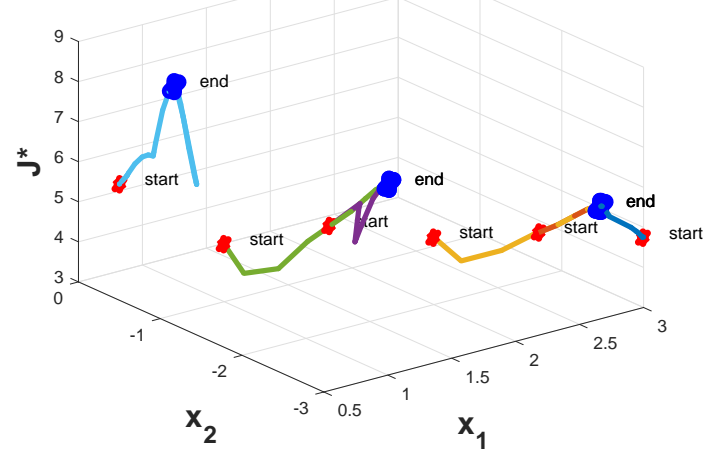

Figure 3.31: Set of position for NOESC-PSO in a 1D case (3 vehicles).

\subsubsection{Conclusion for Sensitivity}

Studying the sensitivity of the PESC or NOESC with PSO to changes in the initial condition in antenna array application showed evident that the PESC method and NOESC-PSO method are equally sensitive. When implementing the PESC and NOESC-PSO, they both converge or diverge to different optimal objective functions depending on the initial condition. With that it can be said that neither NOESC with PSO or PESC is more efficient than the other. 


\section{CHAPTER IV}

\section{D AND 3D FIXED ANTENNA ORIENTATION}

\subsection{Three Vehicles in 2D with Fixed Antenna Orientation}

In this section we consider a two dimension case where three vehicles are carrying directional fixed antennas. The first of the three vehicles is positioned at $\left(x_{1}, y_{1}, 0\right)$, where the whole vehicle is located in the positive quarter. The second is positioned at $\left(x_{2}, y_{2}, 0\right)$, where $x_{2}$ is negative and $y_{2}$ is positive. And the last vehicle has a position of $\left(x_{3}, y_{3}, 0\right)$, where the vehicle is located completely in the negative quarter. Each vehicle has two degrees of freedom, which would be a total of six degrees of freedom. The block diagram represents the three vehicles in $2 \mathbf{D}$ with fixed antenna orientation shown in Figure 4.1. The two algorithms, PESC and NOESC with PSO, were used to see which can get the optimum directivity and optimum position in 2D for the six degrees of freedom. 


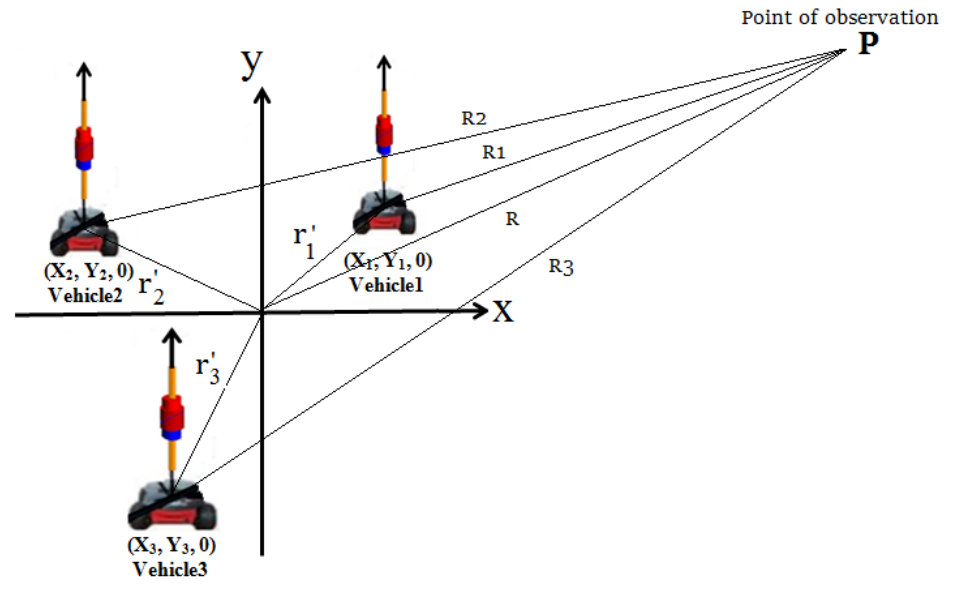

Figure 4.1: Three vehicles in 2D and fixed antenna orientation.

\subsection{D Arbitrary Positioned Antenna Arrays}

An arbitrary positioned antenna array in $2 \mathbf{D}$ has a setup where the dipoles are oriented in $z$-axis and positioned in the $x$ and $y$ axis as shown in Figure 4.1. For two dimensional arrays with elements on the $x-y$ plane the far-field radiation pattern of the array is given by

$$
\begin{aligned}
& F(\theta, \phi) \simeq\left[\left(\sin \theta_{n} \cos \theta\left(\cos \left(\phi-\phi_{n}\right)\right)\right.\right. \\
& \left.\left.\quad-\sin \theta \cos \theta_{n}\right) \operatorname{sinc}\left(\frac{\beta L}{2}\left(\sin \theta_{n} \sin \theta\left(\cos \left(\phi-\phi_{n}\right)\right)+\cos \theta \cos \theta_{n}\right)\right)\right] e^{\left\{j \beta\left(x_{n} \sin \theta \cos \phi+y_{n} \sin \theta \sin \phi\right)\right\}} .
\end{aligned}
$$

\subsubsection{Using Perturbation-based Extremum Seeking Control}

The simulation results can be found in Figure 4.2, Figure 4.3, and Figure 4.4 where the initial conditions are $x_{1}(0)=0.3, y_{1}(0)=0.3, x_{2}(0)=-0.2, y_{2}(0)=0.2, x_{3}(0)=-0.3$, and $y_{3}(0)=-0.3$. The parameters of the perturbation based extremum seeking control simulation are selected as $\omega_{1}=5 \mathrm{rad} / \mathrm{s}, \omega_{2}=6 \mathrm{rad} / \mathrm{s}, \omega_{3}=7 \mathrm{rad} / \mathrm{s}$, loop perturbation amplitude $\alpha_{i}=0.01$, loop 
gain $C_{x_{i}}=C_{y_{i}}=1$, and $i=1,2,3$. The weights of the potential function are chosen as $K=1$. The performance function is increased to a neighborhood of the maximum. It can also be noticed that three different perturbation frequencies are needed for three vehicles in a two-dimensional space. The vehicle starts at the initial condition by probing around to climb the gradient of the unknown map, the output of the unknown signal $J$ is shown in Figure 4.2. The PESC is indeed able to find the optimum directivity $J$ even though the optimum input varies with time as shown in Figure 4.2 . As expected, $J$ converges to a periodic motion around $J^{*}$. It also shows that the objective function was driven toward its optimum rapidly and kept at optimum by the gradient method. The noise that appears before the objective function reaches its optimum value of 8.3364 is caused by the fact that the PESC method is based on the sinusoidal wave perturbation.

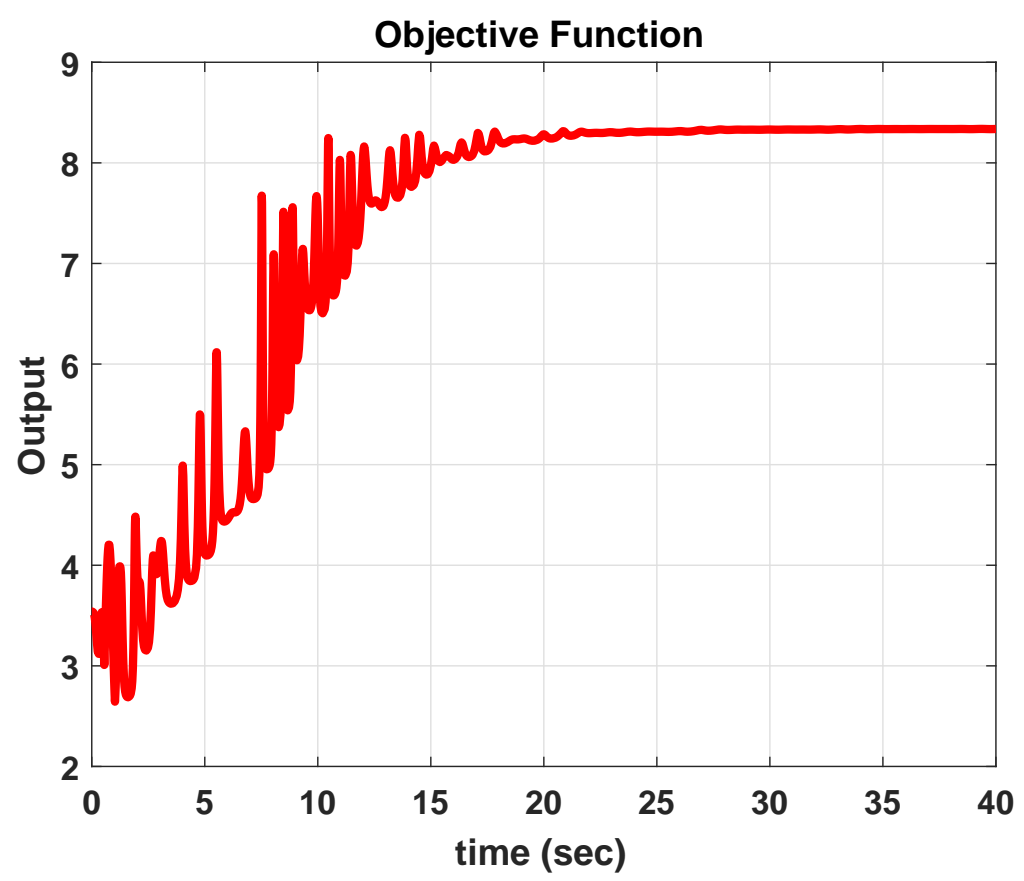

Figure 4.2: Objective function for PESC in a 2D case (3 vehicles). 
Next, we look at Figure 4.3. It indicates that time and position effect each other. Here, we notice that the PESC caused the optimum position to converge to $\left(x_{1}^{*}, y_{1}^{*}\right)=(0.5363,0.1898)$, $\left(x_{2}^{*}, y_{2}^{*}\right)=(1.2658,0.4151)$, and $\left(x_{3}^{*}, y_{3}^{*}\right)=(-0.1946,-0.0321)$. The optimum position of the three $x^{*}$ and $y^{*}$ is the point where the $J$ function reaches the value of $J^{*}$. In this figure we also notice the oscillation again which is caused by the effect of the sine wave used in the PESC.

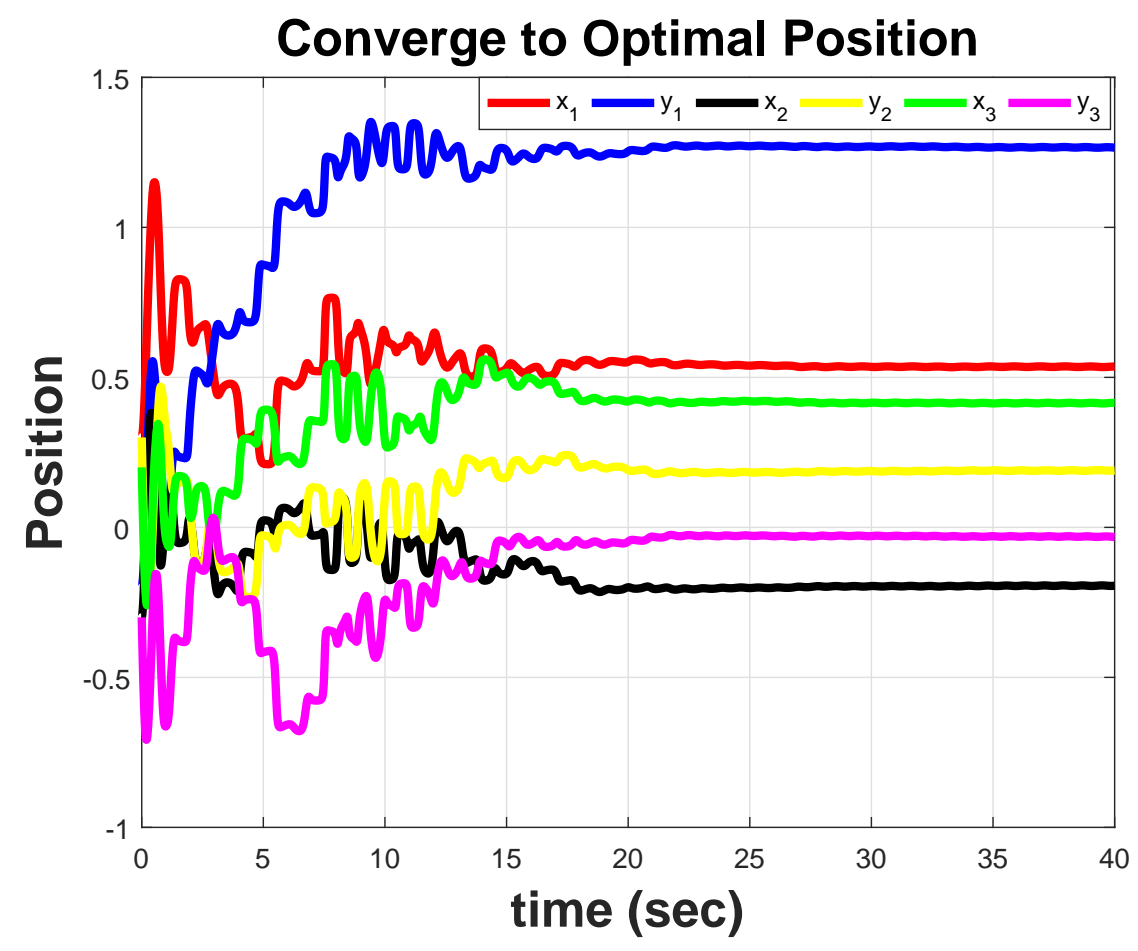

Figure 4.3: Position for PESC in a 2D case (3 vehicles).

The controller can be seen in Figure 4.4 The controller converges to a neighborhood of zero around the optimum values of the objective function and the position. 


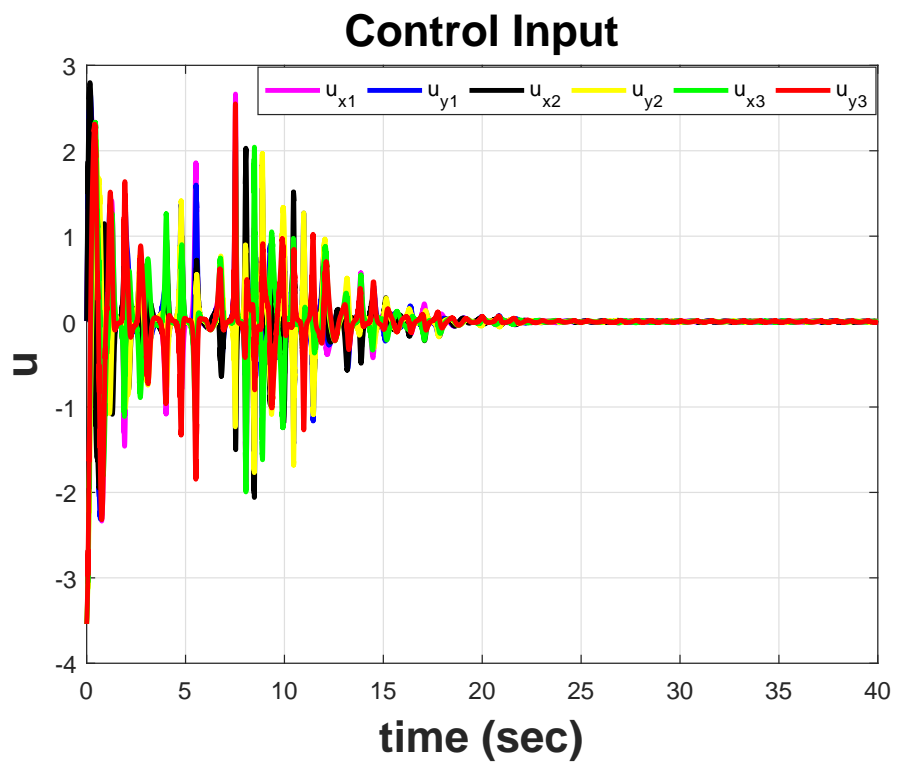

Figure 4.4: Control input of $x$-axis for PESC in a 2D case (3 vehicles).

Figure 4.5 shows the 3D view of how the vehicles' trajectory converges to the optimal objective function.

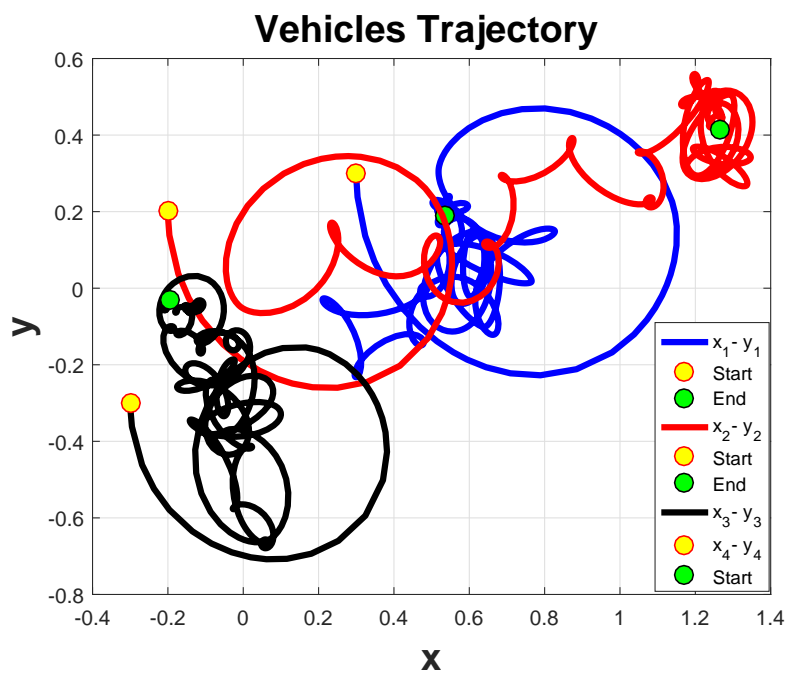

Figure 4.5: Vehicles trajectory for PESC in a 2D case (3 vehicles). 


\subsubsection{Using Numerical Optimization-based Extremum Seeking Control with DS}

Using the same initial condition as before, but instead applying NOESC with DS, the same process was followed. The objective functions was 8.327 as seen in Figure 4.6. It converged at 0.6 seconds meaning that the convergence was fast, which is desirable. In Figure 4.7 the closed-loop system is shown to converge to the correct maximizers $\left(x_{1}^{*}, y_{1}^{*}\right)=(0.4513,0.6172),\left(x_{2}^{*}, y_{2}^{*}\right)=$ $(-0.0857,0.0857)$, and $\left(x_{3}^{*}, y_{3}^{*}\right)=(-0.6172,-0.4530)$. And the controller converged to a neighborhood of zero in Figure 4.8, Figure 4.9 shows the 3D view of how the vehicles' trajectory converges to the optimal objective function.

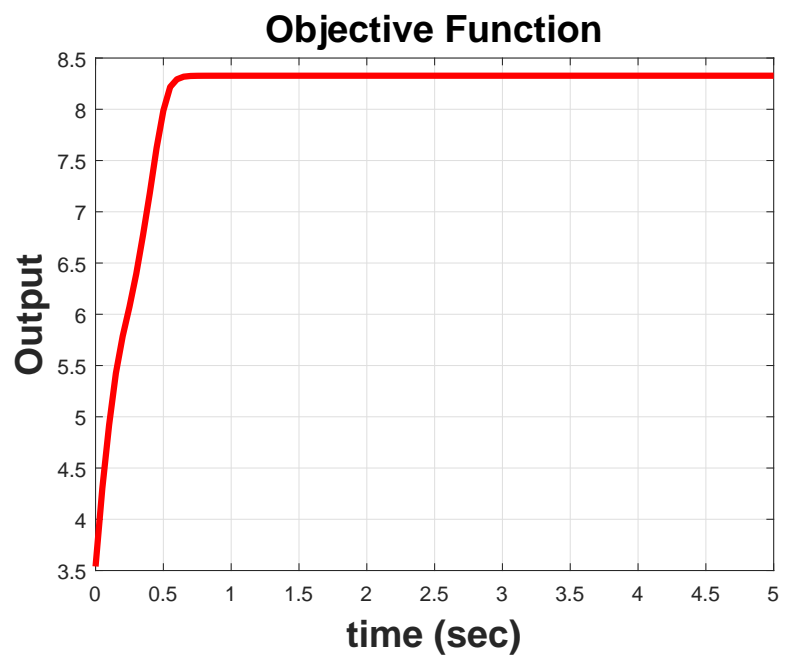

Figure 4.6: Objective function for NOESC-DS in a 2D case (3 vehicles). 


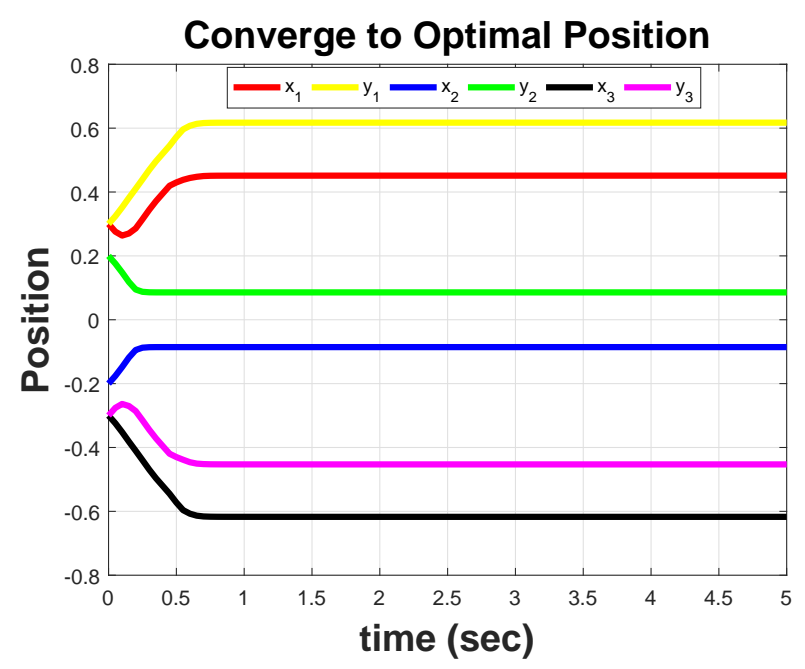

Figure 4.7: Position for NOESC-DS in a 2D case (3 vehicles).

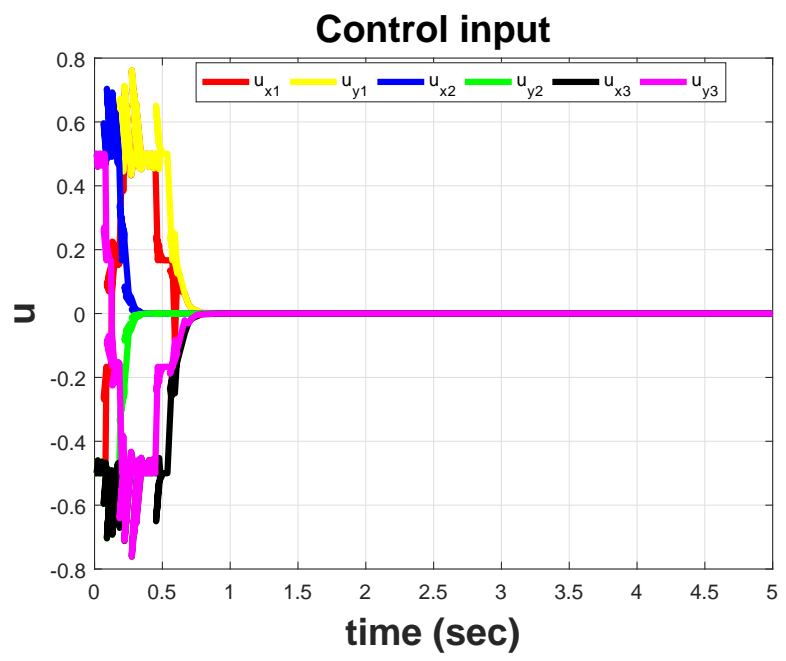

Figure 4.8: Control input of $x$-axis for NOESC-DS in a 2D case (3 vehicles). 


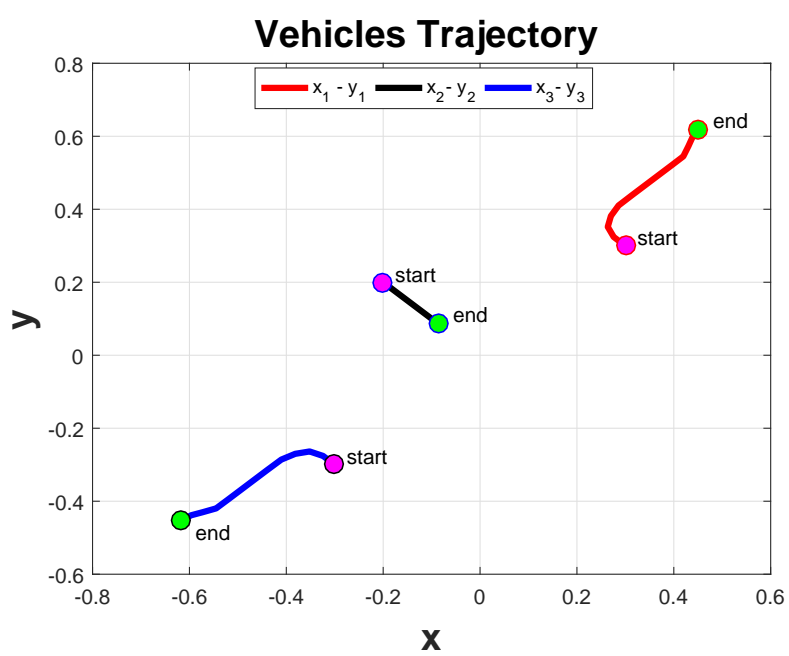

Figure 4.9: Vehicles trajectory for NOESC-DS in a 2D case (3 vehicles).

\subsubsection{Using Numerical Optimization-based Extremum Seeking Control with PSO}

Griddatan, which is a function that supports scattered data interpolation in $N-D$, can have an exponential growth in memory required by the underlying triangulation. Using it produces $\hat{J}$. Only when using PSO, $\hat{J}$ is considered the objective function. Using the same initial condition as before, but instead applying $\hat{J}$ on NOESC with PSO, the same process was followed. The objective functions was 8.2562 as seen in Figure 4.10 . It converged at six seconds meaning that the convergence was fast, which is desirable. In Figure 4.11, the closed-loop system is shown to converge to the correct maximizers $\left(x_{1}^{*}, y_{1}^{*}\right)=(0.0695,0.7537),\left(x_{2}^{*}, y_{2}^{*}\right)=(-0.0508,0.0026)$, and $\left(x_{3}^{*}, y_{3}^{*}\right)=(-0.1708,-0.7495)$. And in Figure 4.12, the controller converged to a neighborhood of zero. Figure 4.13 shows the 3D view of how the vehicles' trajectory converges to the optimal objective function. 


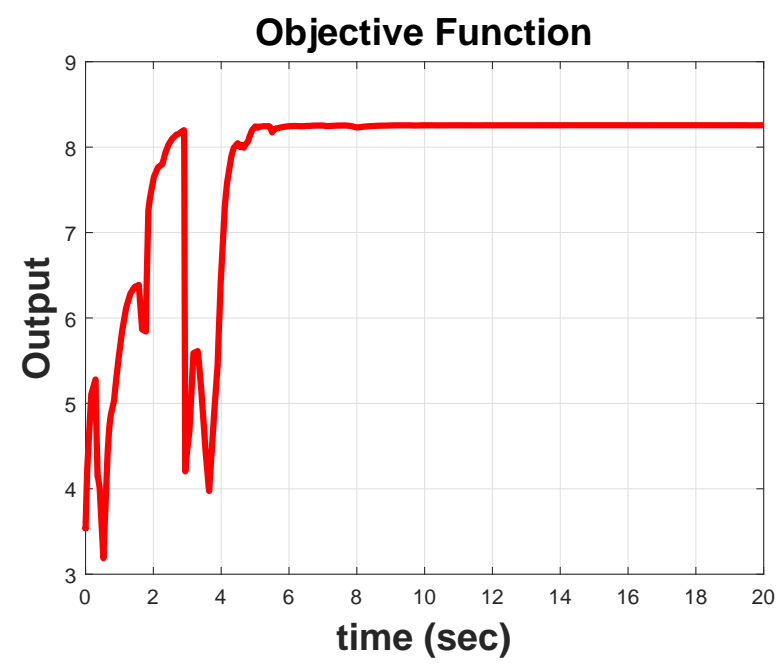

Figure 4.10: Objective function for NOESC-PSO in a 2D case (3 vehicles).

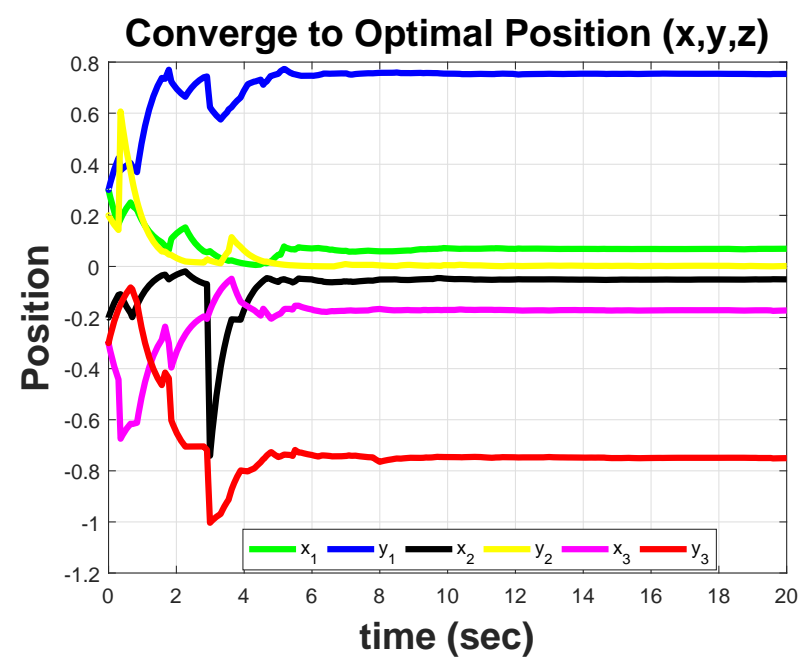

Figure 4.11: Position for NOESC-PSO in a 2D case (3 vehicles). 


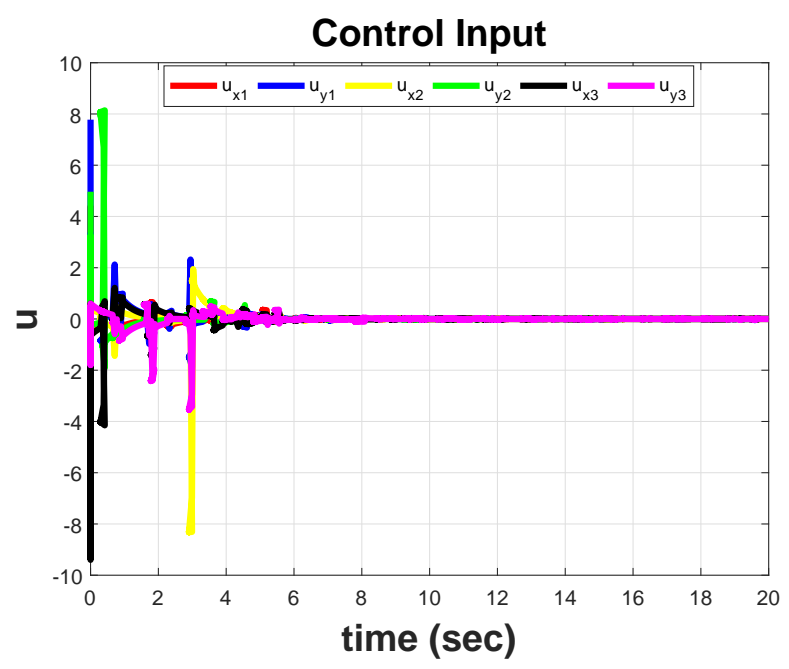

Figure 4.12: Control input of $x$-axis for NOESC-PSO in a 2D case (3 vehicles).

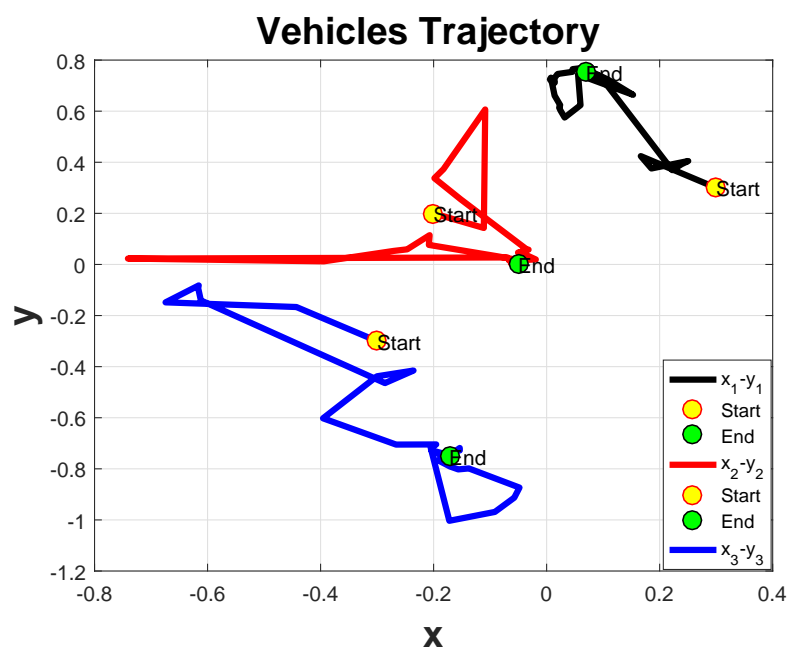

Figure 4.13: Vehicles trajectory for NOESC-PSO in a 2D case (3 vehicles).

\subsubsection{Comparison Results of PESC and NOESC with DS and PSO}

The three algorithms, PESC, NOESC with DS, and NOESC with PSO, though each is different, obtained similar results. The results can be seen in Table 4.1. The first being that PESC, obtains the 
final objective function $J^{*}=8.3364$, the objective function of the NOESC with DS method obtains $J^{*}=8.327$, and the objective function of the NOESC with PSO method obtains $J^{*}=8.2562$. This shows that they all acquired similar results. The second difference is the convergence time for NOESC with DS is faster than the PESC and NOESC with PSO, which makes NOESC with DS more desirable. The table below shows the differences.

Table 4.1: Comparison results of three algorithms in a 2D case (3 vehicles).

\begin{tabular}{|c|c|c|}
\hline $\mathrm{ESC}$ & Optimum objective & Optimum position \\
\hline PESC & 8.3364 & $\begin{array}{l}\left(x_{1}^{*}, y_{1}^{*}\right)=(0.5363,0.1898) \\
\left(x_{2}^{*}, y_{2}^{*}\right)=(1.2658,0.4151) \\
\left(x_{3}^{*}, y_{3}^{*}\right)=(-0.1946,-0.0321)\end{array}$ \\
\hline NOESC-DS & 8.327 & $\begin{array}{l}\left(x_{1}^{*}, y_{1}^{*}\right)=(0.4513,0.6172) \\
\left(x_{2}^{*}, y_{2}^{*}\right)=(-0.0857,0.0857) \\
\left(x_{3}^{*}, y_{3}^{*}\right)=(-0.0172,-0.4530)\end{array}$ \\
\hline NOESC-PSO & 8.2562 & $\begin{array}{l}\left(x_{1}^{*}, y_{1}^{*}\right)=(0.6695,0.7537) \\
\left(x_{2}^{*}, y_{2}^{*}\right)=(-0.0508,0.0026) \\
\left(x_{3}^{*}, y_{3}^{*}\right)=(-0.1708,-0.7495)\end{array}$ \\
\hline
\end{tabular}

\subsection{Four Vehicles in 3D with Fixed Antenna Orientation}

The extremum seeking case with point mass vehicle dynamics can be extended to the three dimensional case. The first vehicle located in the positive quarter has a coordinate of $\left(x_{1}, y_{1}, z_{1}\right)$. With the coordinates of $\left(x_{2}, y_{2}, z_{2}\right)$, the second vehicle is located with $x_{2}$ being negative, and $y_{2}$ and $z_{2}$ being positive. The third vehicle with $x_{3}$ and $y_{3}$ negative and $z_{3}$ positive, has a coordinate of $\left(x_{3}, y_{3}, z_{3}\right) . x_{4}$ and $z_{4}$ are positive, and the $y_{4}$ is negative, giving the coordinates $\left(x_{4}, y_{4}, z_{4}\right)$, for the fourth vehicle. In this case, the total radiation pattern of the fixed antenna orientation is defined by 
Equation 2.11. PESC and NOESC with PSO were the two algorithms implemented to the system.

The diagram of the vehicles is shown in Figure 4.14

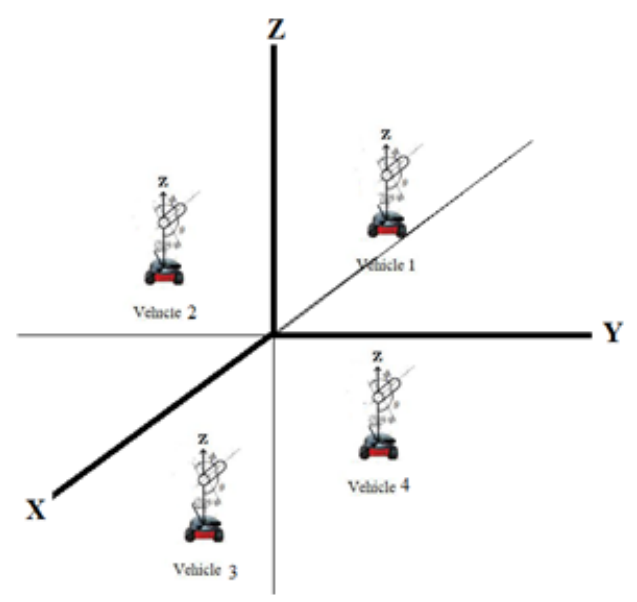

Figure 4.14: Four vehicles in 3D and fixed antenna orientation.

\subsubsection{Using Perturbation-based Extremum Seeking Control}

The simulation results can be found in Figures 4.15 . 4.18 where the initial conditions are $\left(x_{1}(0)\right)=0.81,\left(y_{1}(0)\right)=0.21,\left(z_{1}(0)\right)=1.25,\left(x_{2}(0)\right)=-0.43,\left(y_{2}(0)\right)=0.31,\left(z_{2}(0)\right)=$ $0.18,\left(x_{3}(0)\right)=-0.44,\left(y_{3}(0)\right)=-0.35,\left(z_{3}(0)\right)=0.15,\left(x_{4}(0)\right)=0.8,\left(y_{4}(0)\right)=-0.45$ and $\left(z_{4}(0)\right)=1.24$. The parameters of the perturbation based extremum seeking control simulation are selected as the perturbation frequency $\omega_{1}=21 \mathrm{rad} / \mathrm{s}, \omega_{2}=22 \mathrm{rad} / \mathrm{s}, \omega_{3}=23 \mathrm{rad} / \mathrm{s}$ and $\omega_{4}=24 \mathrm{rad} / \mathrm{s}$, perturbation amplitude $\alpha_{i}=0.001$, adaptation gains $C_{x_{i}}=C_{y_{i}}=C_{z_{i}}=1$, $i=1,2,3,4$ and washout filter chosen as $h=1$. The performance function is increased to a neighborhood of the maximum. It can also be noticed that four different perturbation frequencies are needed for four vehicles in a three-dimensional space. The vehicle starts at the initial condition by probing around to climb the gradient of the unknown map, the output of the unknown 
signal $J$ is shown in Figure 4.15. Figure 4.15 shows that the PESC is indeed able to find the optimum directivity $J$ even though the optimum input varies with time. As expected, $J$ converge to a periodic motion around $J^{*}$. It also shows that the objective function was driven toward its optimum rapidly and kept at optimum by the gradient method. The noise that appears before the objective function reaches its optimum value of 11.6415 is caused by the fact that the Perturbation-based Extremum Seeking Control method is based on the sinusoidal wave perturbation. The PESC showed good results because it was able to find the optimal position for four vehicles, which is $\left(x_{1}^{*}, y_{1}^{*}, z_{1}^{*}\right)=(1.3282,0.0427,1.7682),\left(x_{2}^{*}, y_{2}^{*}, z_{2}^{*}\right)=(0.0780,0.1563,0.6880)$, $\left(x_{3}^{*}, y_{3}^{*}, z_{3}^{*}\right)=(0.0620,-0.5067,0.6520)$, and $\left(x_{4}^{*}, y_{4}^{*}, z_{4}^{*}\right)=(1.3101,-0.6182,1.7501)$, as seen in Figure 4.16. In Figure 4.17, the controller was able to converge to a neighborhood of zero. Figure 4.18 shows the 3D trajectories of the vehicles converging into the objective function in 3D.

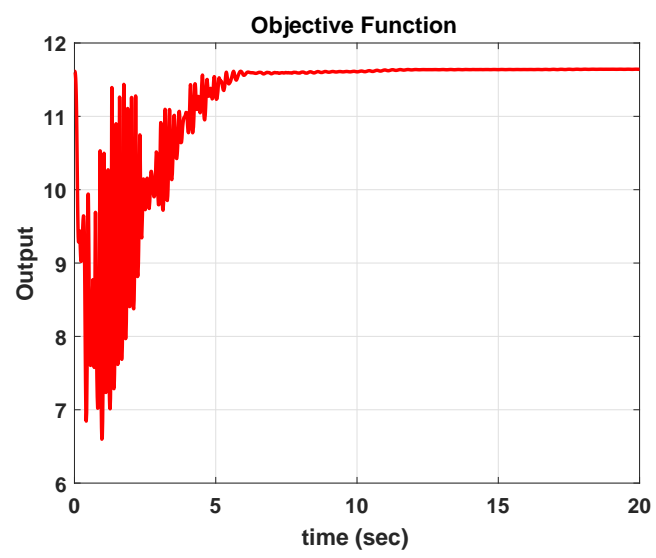

Figure 4.15: Objective function for PESC in a 4 vehicles in 3D. 


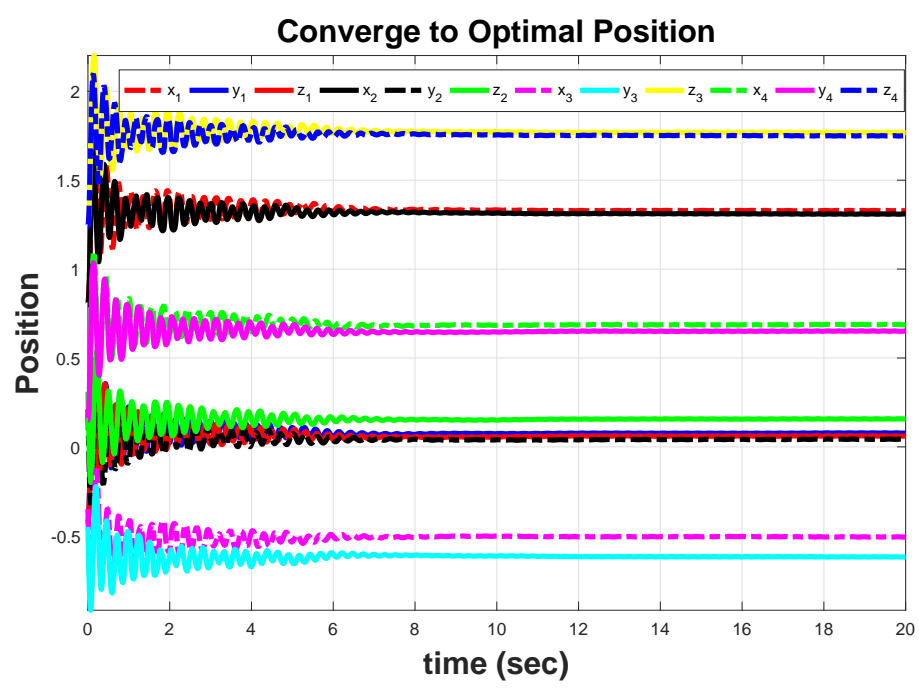

Figure 4.16: Position for PESC in a 4 vehicles in 3D.

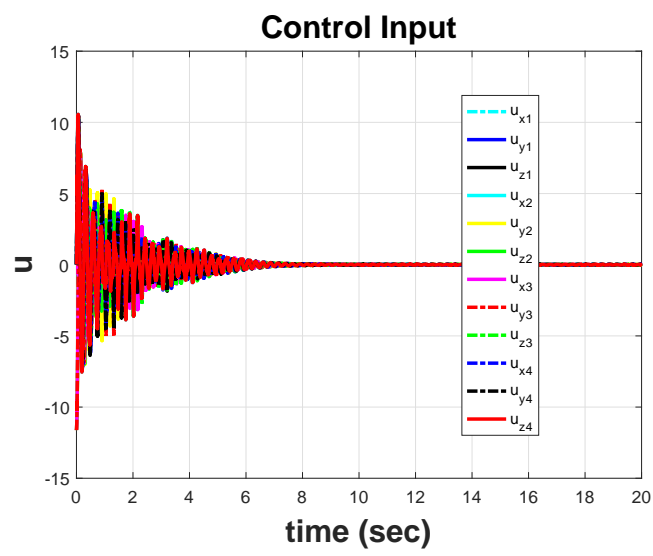

Figure 4.17: Control input for PESC in a 4 vehicles in 3D. 


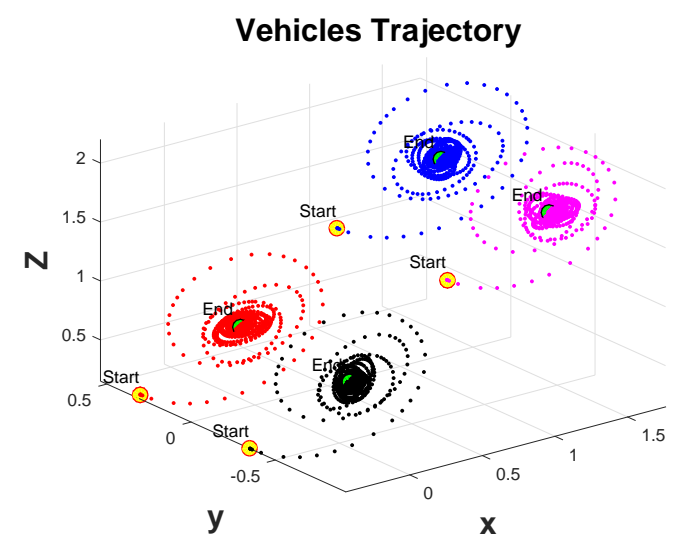

Figure 4.18: Trajectory for for PESC in a 4 vehicles in 3D.

\subsubsection{Using Numerical Optimization-based Extremum Seeking Control with DS}

With there being four vehicles each having a coordinate of $x, y$, and $z$, there is 12 degrees of freedom when applying NOES-DS. While maintaining the same initial condition as PESC, the same procedure is done. The objective functions was 11.6410, which can be seen in Figure 4.19. It converged at two seconds meaning that the convergence was fast, which is desirable. The closed-loop system is shown to converge to the correct maximizers $\left(x_{1}^{*}, y_{1}^{*}, z_{1}^{*}\right)=(0.8123,0.2140,1.2568)$, $\left(x_{2}^{*}, y_{2}^{*}, z_{2}^{*}\right)=(-0.4331,0.3118,0.1754),\left(x_{3}^{*}, y_{3}^{*}, z_{3}^{*}\right)=(-0.4423,-0.3534,0.1477)$, and $\left(x_{4}^{*}, y_{4}^{*}, z_{4}^{*}\right)=(0.8031,-0.4518,1.2382)$ in Figure 4.20 . The controller converged to a neighborhood of zero as seen in Figure 4.21. Than in Figure 4.22 shows the 3D view of how the vehicles' trajectory converges to the optimal objective function. 


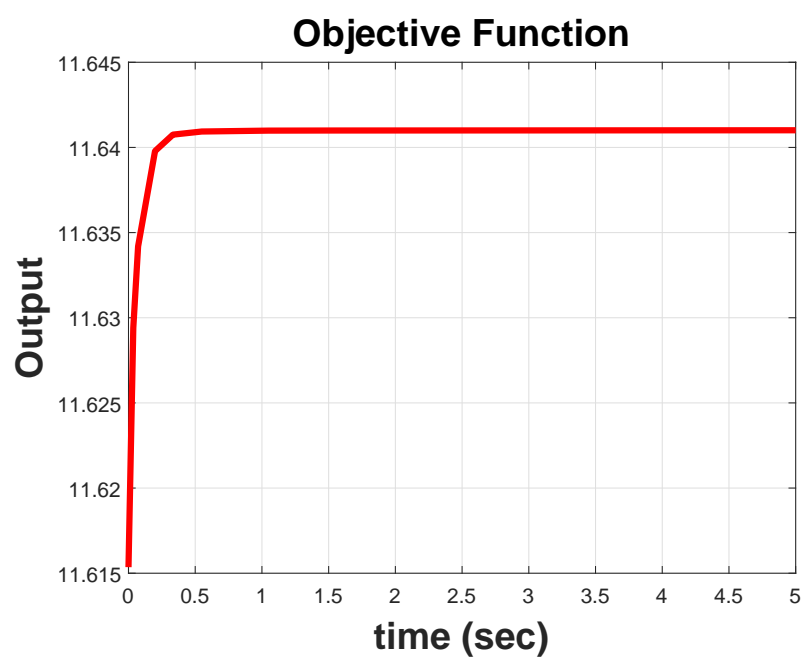

Figure 4.19: Objective function for NOESC-DS for 3D case (4 vehicles).

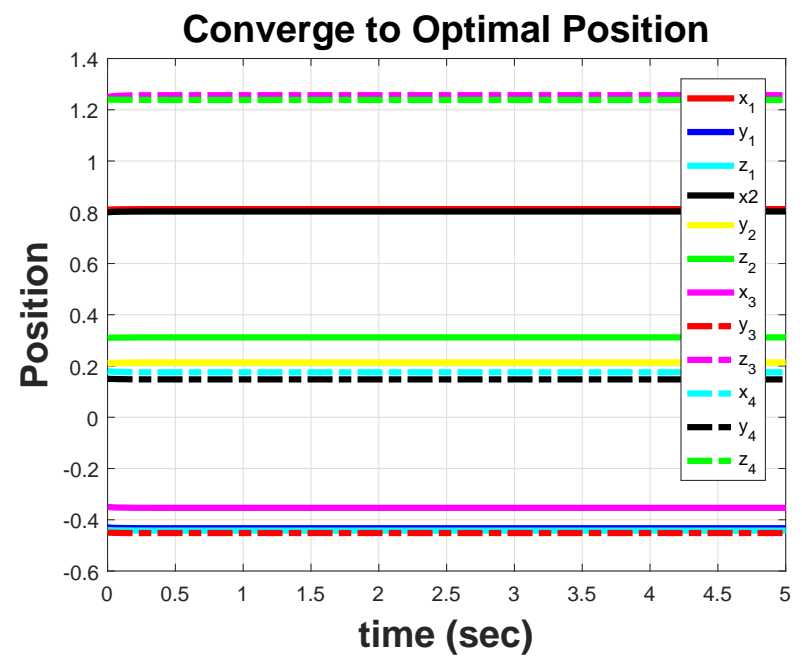

Figure 4.20: Position for NOESC-DS for 3D case (4 vehicles). 


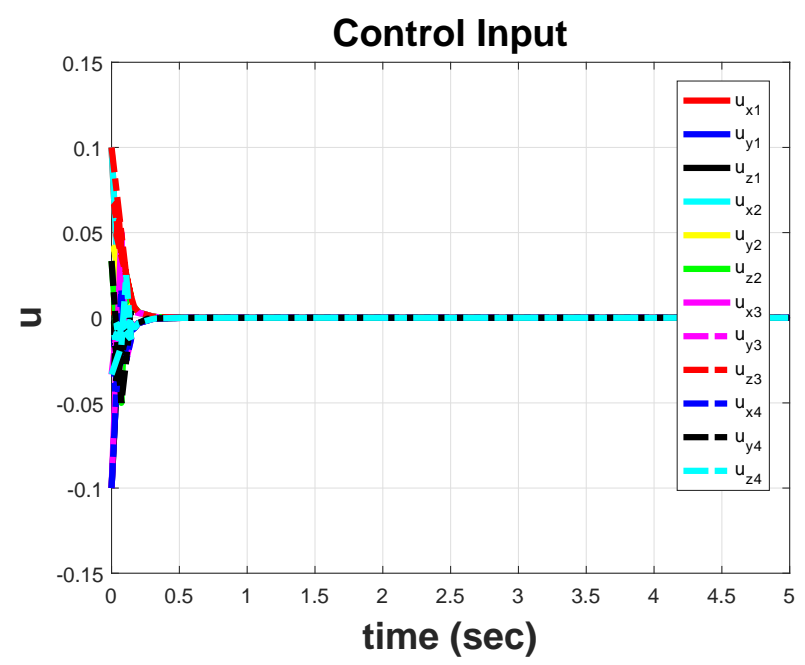

Figure 4.21: Control input of $x$-axis for NOESC-DS for 3D case (4 vehicles).

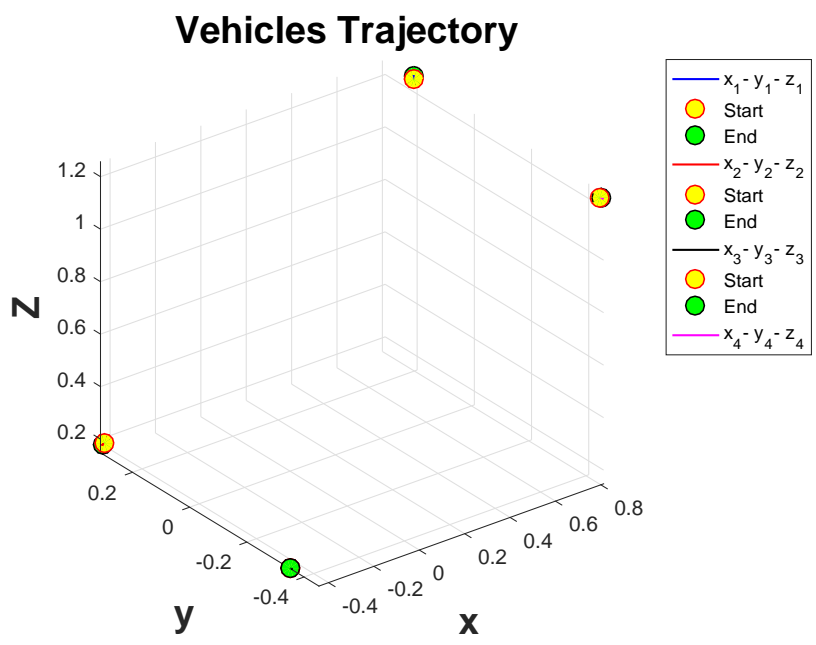

Figure 4.22: Vehicles trajectory for NOESC-DS for 3D case (4 vehicles).

\subsubsection{Comparison of Results of PESC and NOESC-DS}

There is one main differences in the results of PESC and NOESC with DS. The difference is that the convergence time for NOESC with DS is faster than the PESC, which makes NOESC with 
DS more desirable. The the PESC produces the objective function value $J^{*}=11.6415$, and the objective function of the NOESC with DS method yields $J^{*}=11.6410$, meaning they are quite similiar. 


\section{CHAPTER V}

\section{D CASE WITH ARBITRARY ANTENNA ORIENTATION}

\subsection{N Vehicles in 3D with Arbitrary Antenna Orientation}

In order to improve the performance of the transmitter section, multiple independent radiating elements can be arranged into a certain geometrical configuration. These antenna configurations are usually referred to as arrays. To control the shape and the directivity of the array pattern, the position of each element is changed. The array pattern control also conceives the possibility of simultaneously steering the main beam in a certain direction. The behavior of the array pattern mainly depends on five parameters:

1. Geometry, i.e. the spatial configuration of the array.

2. Spacing between the multiple elements.

3. Element pattern of the radiators.

4. Amplitude excitation of each element.

5. Phase excitation of each element.

In this thesis, the amplitude and phase excitation of each element are not studied or focused herein. By considering the geometry illustrated in Figure 5.1 that represents a generic distribution and composition of array elements. The basic setup of an arbitrary antenna array is shown in Figure 5.1. The location of the $n^{\text {th }}$ antenna element is described by as following: 


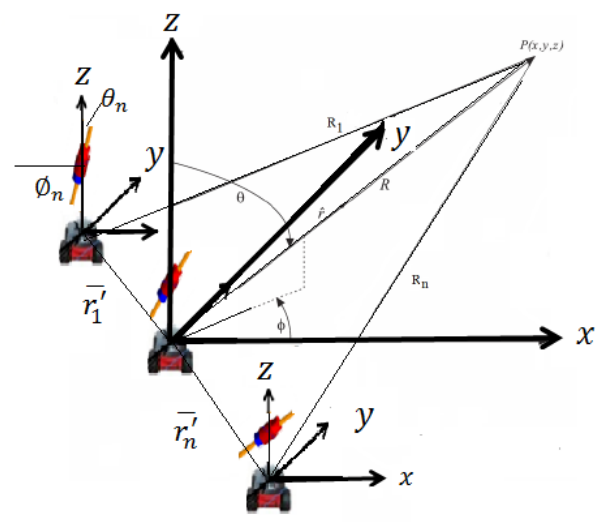

Figure 5.1: Geometry of $\mathrm{N}$ vehicles in 3D for non-uniformly spaced and orientation ntenna array

Equation 5.1 is a general case explaining the $n$-th element and $n$-th position in $3 \mathbf{D}$

$$
\begin{aligned}
& F(\theta, \phi) \simeq\left[\left(\sin \theta_{n} \cos \theta\left(\cos \left(\phi-\phi_{n}\right)\right)\right.\right. \\
& \left.\left.\quad-\sin \theta \cos \theta_{n}\right) \operatorname{sinc}\left(\frac{\beta L}{2}\left(\sin \theta_{n} \sin \theta\left(\cos \left(\phi-\phi_{n}\right)\right)+\cos \theta \cos \theta_{n}\right)\right)\right] e^{\left\{j \beta\left(x_{n} \sin \theta \cos \phi+y_{n} \sin \theta \sin \phi+z_{n} \cos \theta\right)\right\}} .
\end{aligned}
$$

\subsection{TWO Vehicles in 3D with Arbitrary Antenna Orientation}

In this section we consider a three dimension case where $N$ vehicles are carrying directional orientation antennas. The $N$ vehicles will be studied from a two vehicle perspective. It will be tested using two algorithms, PESC and NOESC-PSO. We will study the five degrees of freedom, which are three degrees for the position $(x, y, z)$ and two degrees for the orientation $(\theta, \phi)$. The system should be able to test every possible value for these five parameters to dynamically choose the position and orientation that maximize the directivity in real time.

\subsubsection{Using Perturbation-based Extremum Seeking Control}

Starting with PESC for 2 vehicles in 3D, the parameters are $\omega_{1}=6 \mathrm{rad} / \mathrm{s}, \omega_{2}=7 \mathrm{rad} / \mathrm{s}$, perturbation amplitude $\alpha_{i}=0.01$, adaptation gains $C_{x_{i}}=C_{y_{i}}=C_{z_{i}}=1$, and $i=1,2$, and 
the initial conditions are $x_{1}(0)=0.3, y_{1}(0)=0.3, z_{1}(0)=0.3 x_{2}(0)=-0.2, y_{2}(0)=0.2$, $z_{2}(0)=0.2, \theta_{1}(0)=10^{\circ}, \phi_{1}(0)=10^{\circ}, \theta_{2}(0)=10^{\circ}, \phi_{2}(0)=10^{\circ}$. The performance function is increased to a neighborhood of the maximum. It can also be noticed that two different perturbation frequencies are needed for two vehicles in a three-dimensional space. The vehicle starts at the initial condition by exploring around to scale the gradient of the unknown map, the output of the unknown signal $J$ is shown in Figure 5.2. The PESC is indeed able to obtain the optimum directivity $J$ even though the optimum input varies with time as shown in Figure 5.2. As expected, $J$ converges to a periodic motion around $J^{*}$. It also shows that the objective function was driven toward its optimum rapidly and kept at the optimum by the gradient method. The noise that appears before the objective function reaches its optimum value of 4.8448 is caused by the fact that the PESC method is based on the sinusoidal wave perturbation. Next, we look at Figure 5.3. Here, we notice that the PESC caused the optimum position to converge to $\left(x_{1}^{*}, y_{1}^{*}, z_{1}^{*}\right)=(0.6879,0.4795,0.6879)$ and $\left(x_{2}^{*}, y_{2}^{*}, z_{2}^{*}\right)=(0.2869,-0.0426,0.6869)$. The optimum position of the two $x^{*}$ and $y^{*}$ is the point where the $J$ function reaches the value of $J^{*}$. In this figure we also notice the oscillation again which is caused by the effect of the sine wave used in the PESC. The antenna orientation converged to the optimal angles of $\left(\theta_{1}^{*}, \phi_{1}^{*}\right)=\left(10.3869^{\circ}, 10.1795^{\circ}\right)$ and $\left(\theta_{2}^{*}, \phi_{2}^{*}\right)=\left(10.4869^{\circ}, 9.7574^{\circ}\right)$ as seen in Figure 5.4. The controller input for the position is shown in Figure 5.5, and the controller input for the angles can be seen in Figure 5.6. The controller converges to a neighborhood of zero. And the Figure 5.7 presented the vehicles' trajectory. 


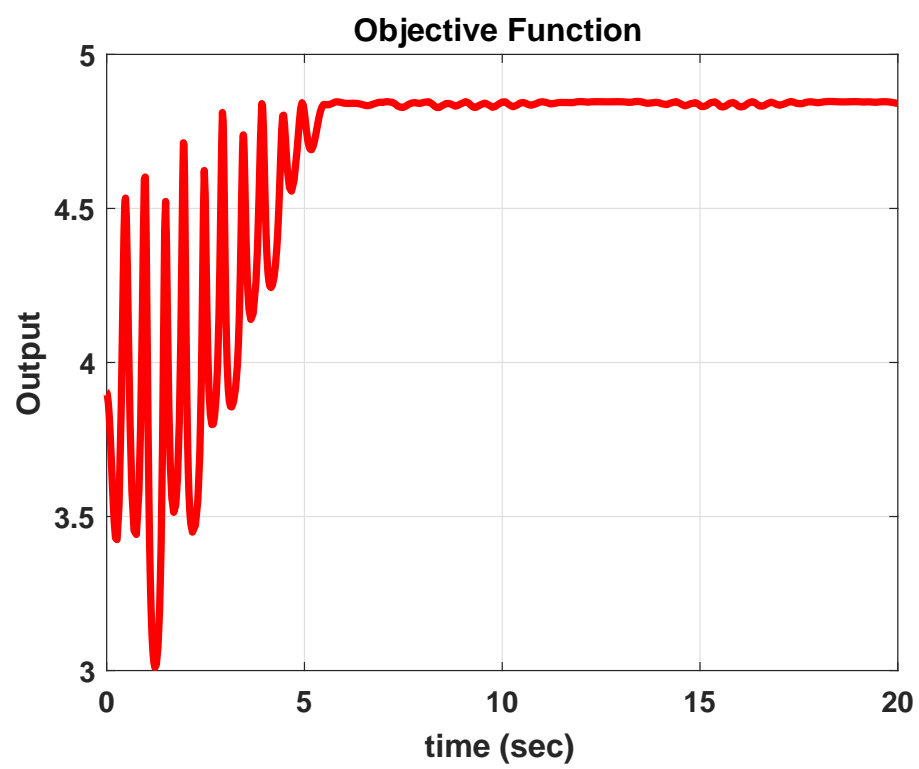

Figure 5.2: Objective function of two vehicles in 3D antenna orientated (PESC).

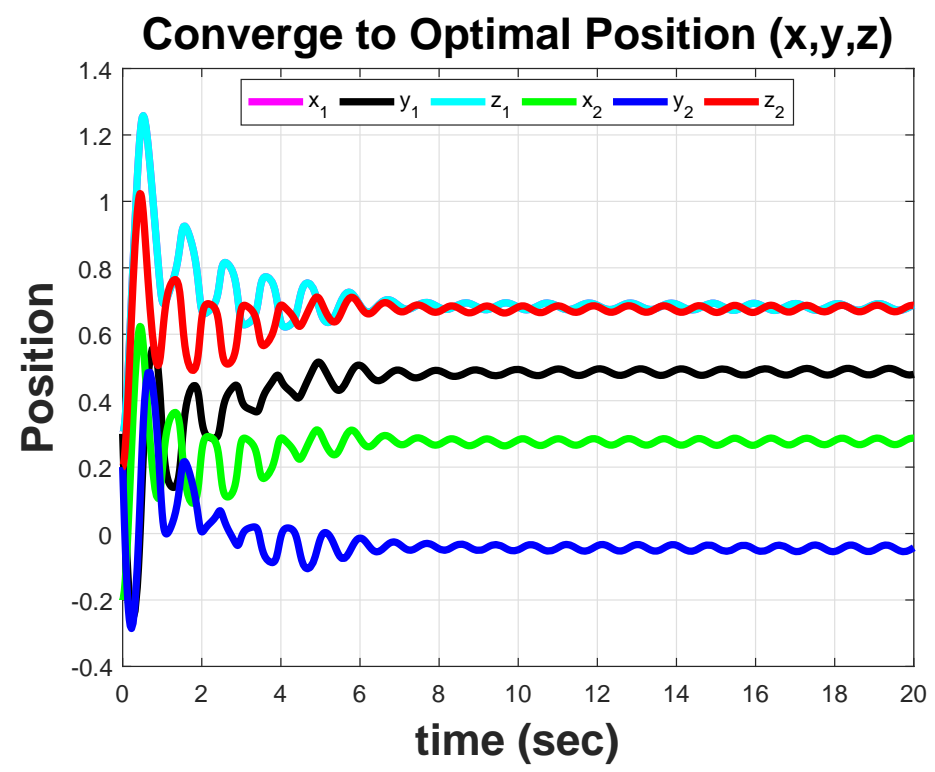

Figure 5.3: Optimal positions of two vehicles in 3D antenna orientated (PESC). 


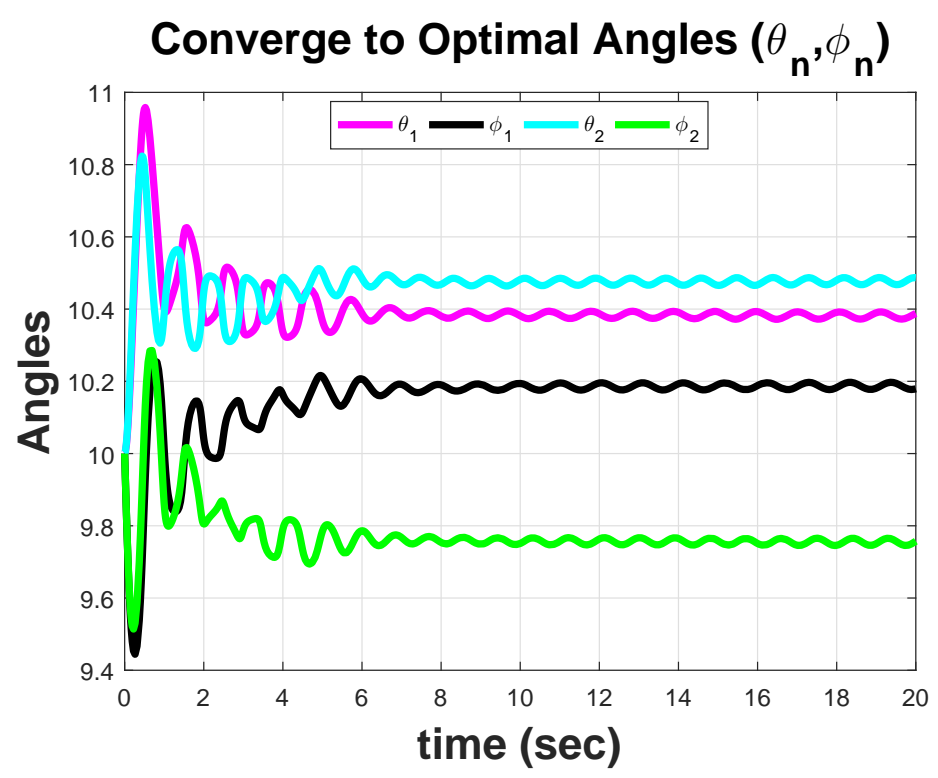

Figure 5.4: Optimal angles of two vehicles in 3D antenna orientated (PESC).

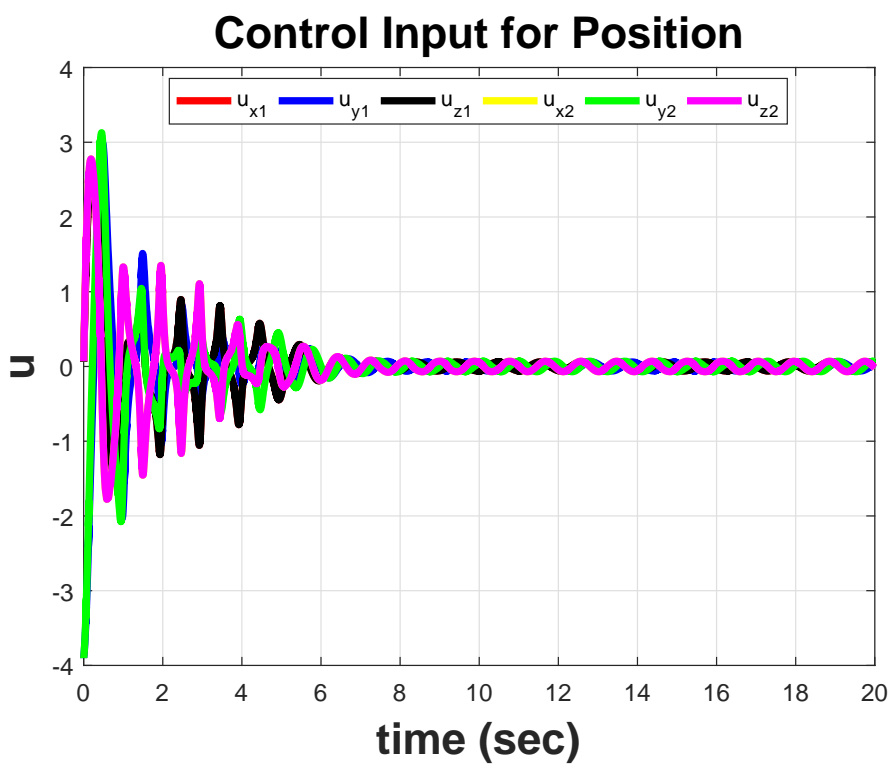

Figure 5.5: Control input for positions of two vehicles in 3D antenna orientated (PESC). 


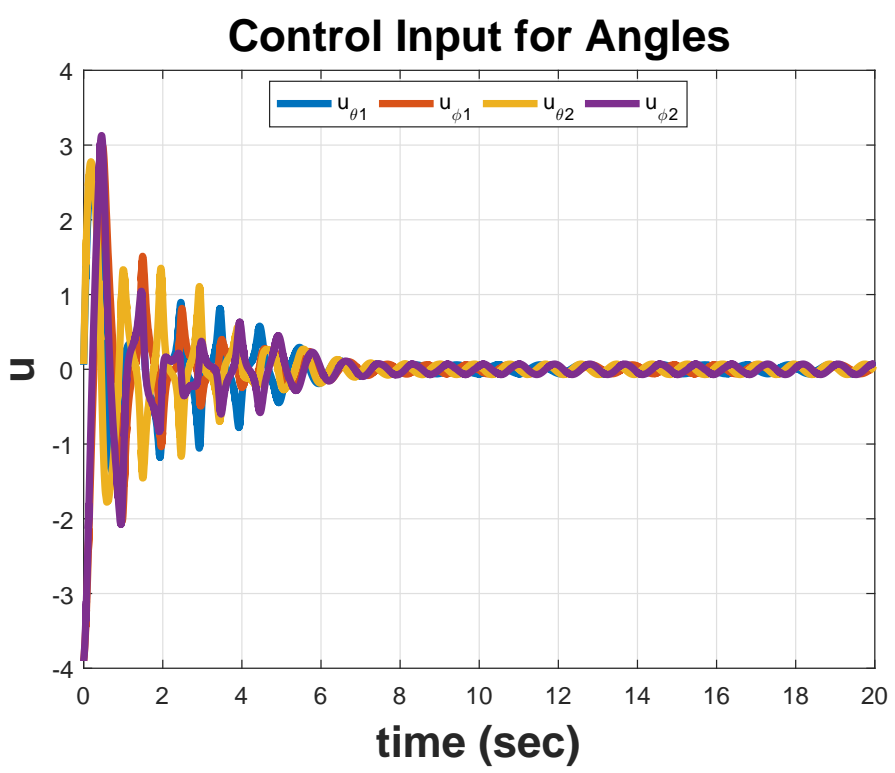

Figure 5.6: Control input for angles of two vehicles in 3D antenna orientated (PESC).

\section{Vehicles Trajectory}

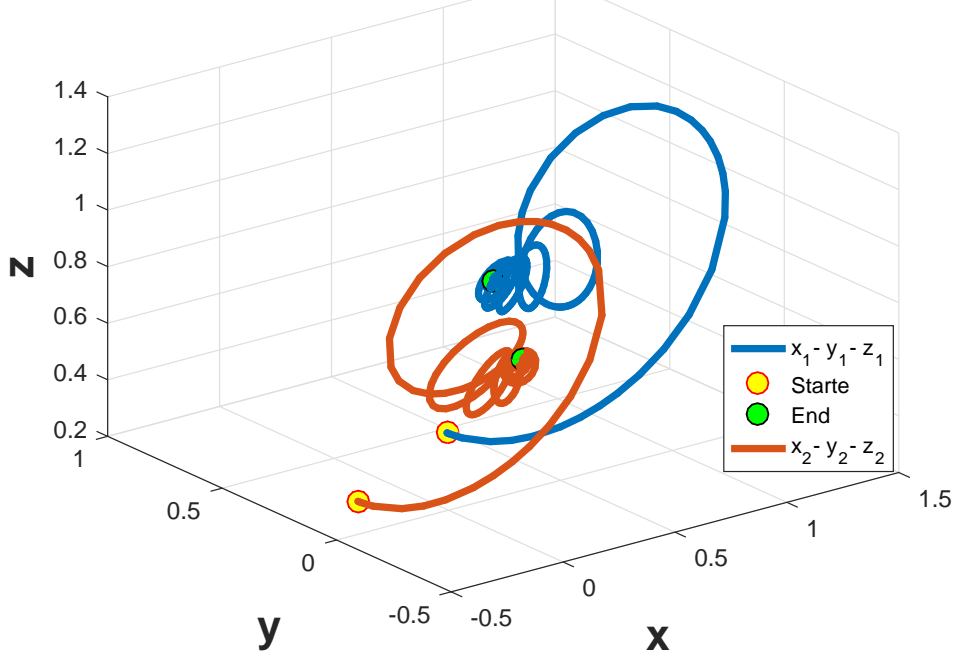

Figure 5.7: Vehicle trajectory for two vehicles in 3D antenna orientated (PESC). 


\subsubsection{Using Numerical Optimization-based Extremum Seeking Control with DS}

When NOESC-DS is used, the initial condition, parameters, and procedure from PESC are maintained. There are ten degrees of freedom because there are two vehicles each having a position of $(x, y, z)$ and orientation of $(\theta, \phi)$. When using NOESC-DS, the objective function outcome was 4.853 , and it converged at 0.65 , as can be seen in Figure 5.8 The closed-loop system is shown to converge to the correct maximizers $\left(x_{1}^{*}, y_{1}^{*}, z_{1}^{*}\right)=(0.3598,0.3589,0.2477)$ and $\left(x_{2}^{*}, y_{2}^{*}, z_{2}^{*}\right)=(-0.2598,0.1411,0.2523)$, shown in Figure 5.9. The antenna orientation converged to the optimal angles of $\left(\theta_{1}^{*}, \phi_{1}^{*}\right)=\left(10.0020^{\circ}, 10.0002^{\circ}\right)$ and $\left(\theta_{2}^{*}, \phi_{2}^{*}\right)=\left(10.0020^{\circ}, 10.0002^{\circ}\right)$ as seen in Figure 5.10. The controller input for position (Figure 5.11) and the controller input for the angles (Figures 5.12) both converged to a neighborhood of zero. And the vehicles trajectory is presented in Figure 5.13 .

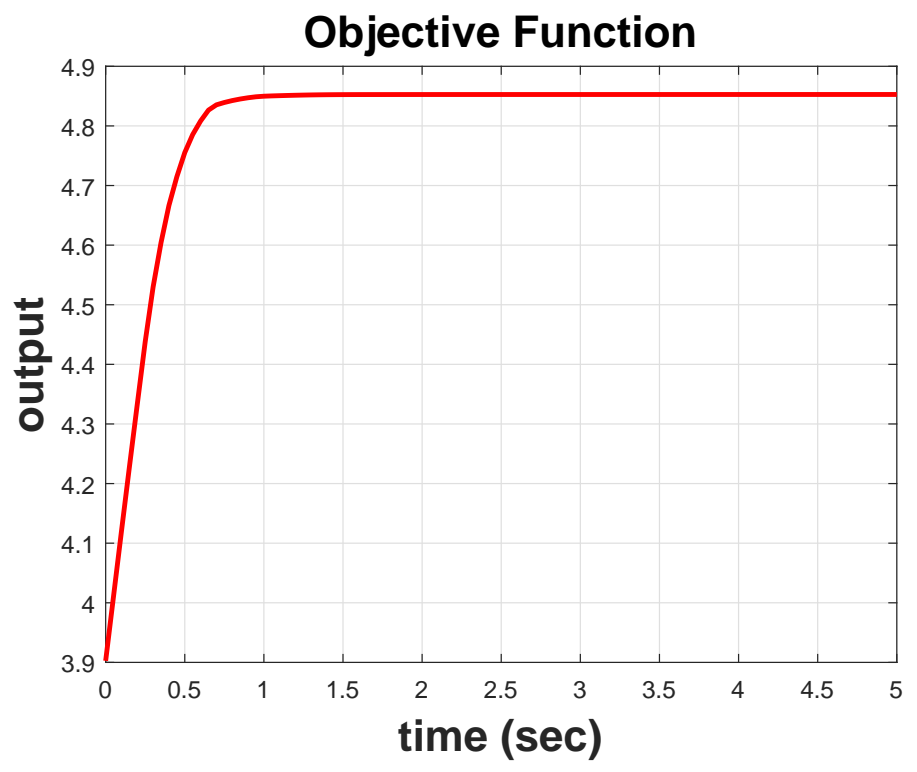

Figure 5.8: Objective function of two vehicles in 3D antenna orientated (NOESC-DS). 


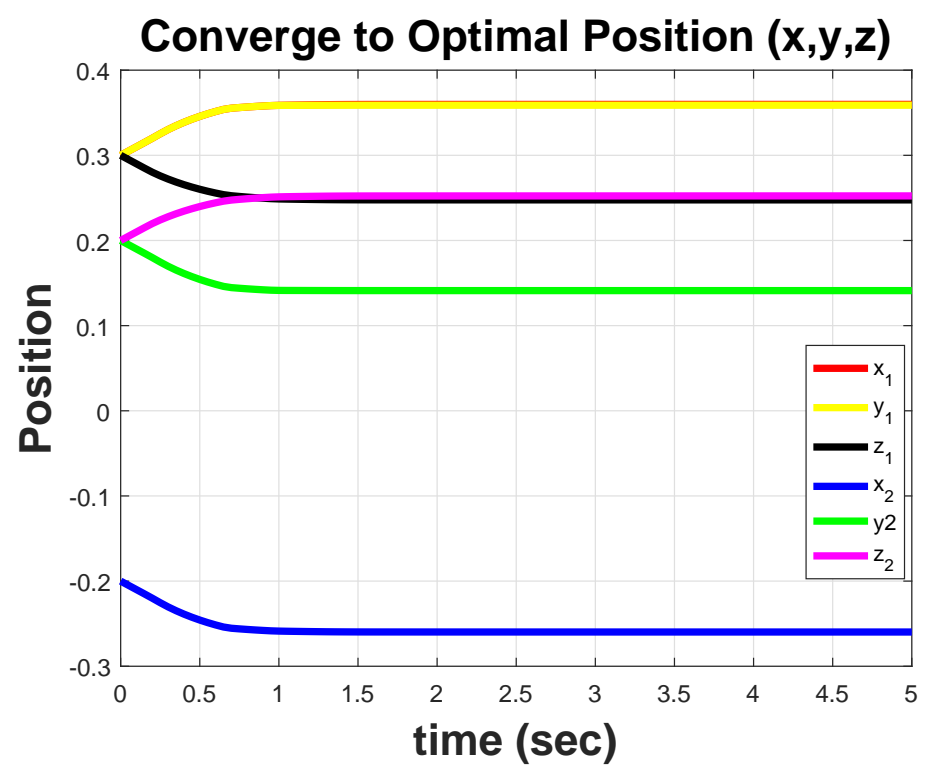

Figure 5.9: Optimal positions of two vehicles in 3D antenna orientated (NOESC-DS).

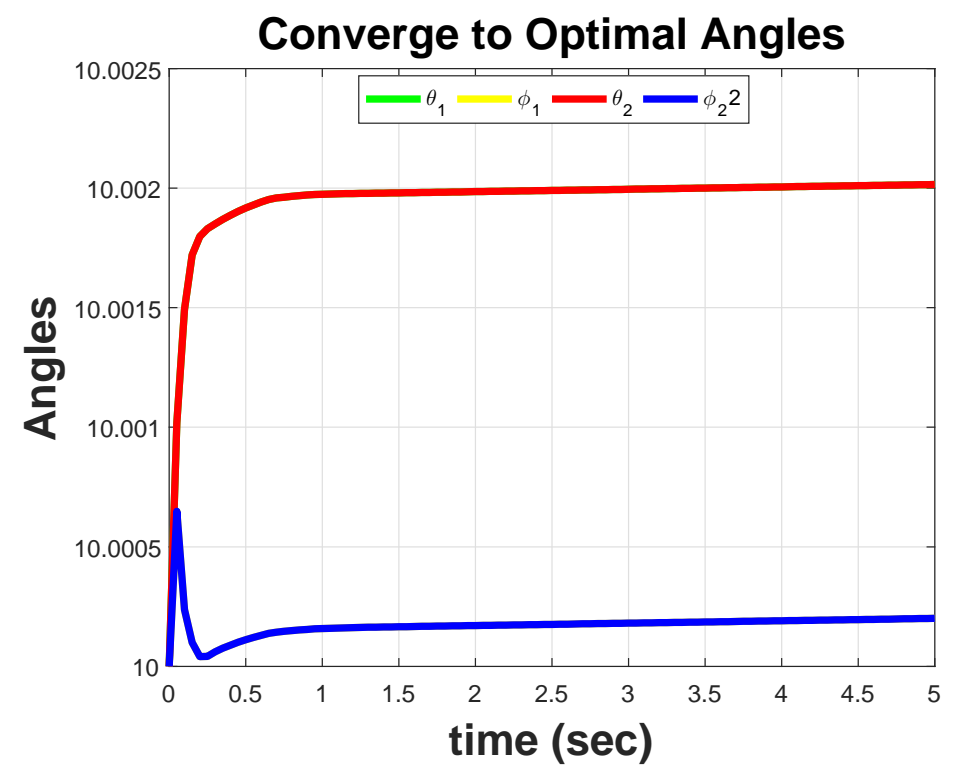

Figure 5.10: Optimal Angles of two vehicles in 3D antenna orientated (NOESC-DS). 


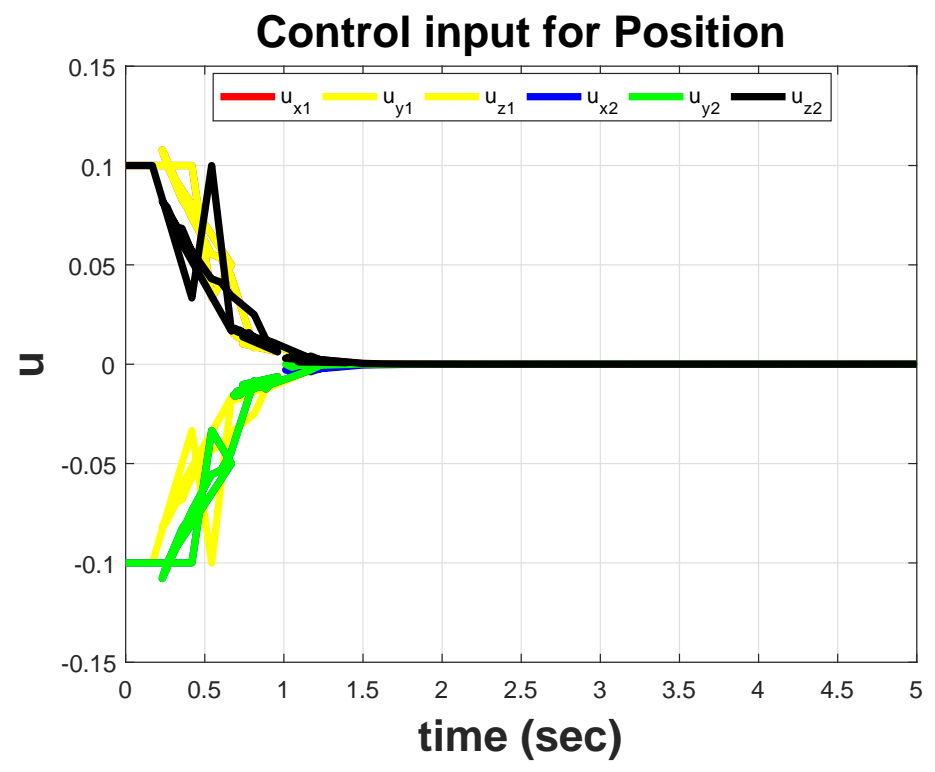

Figure 5.11: Control input for positions of two vehicles in 3D antenna orientated (NOESC-DS).

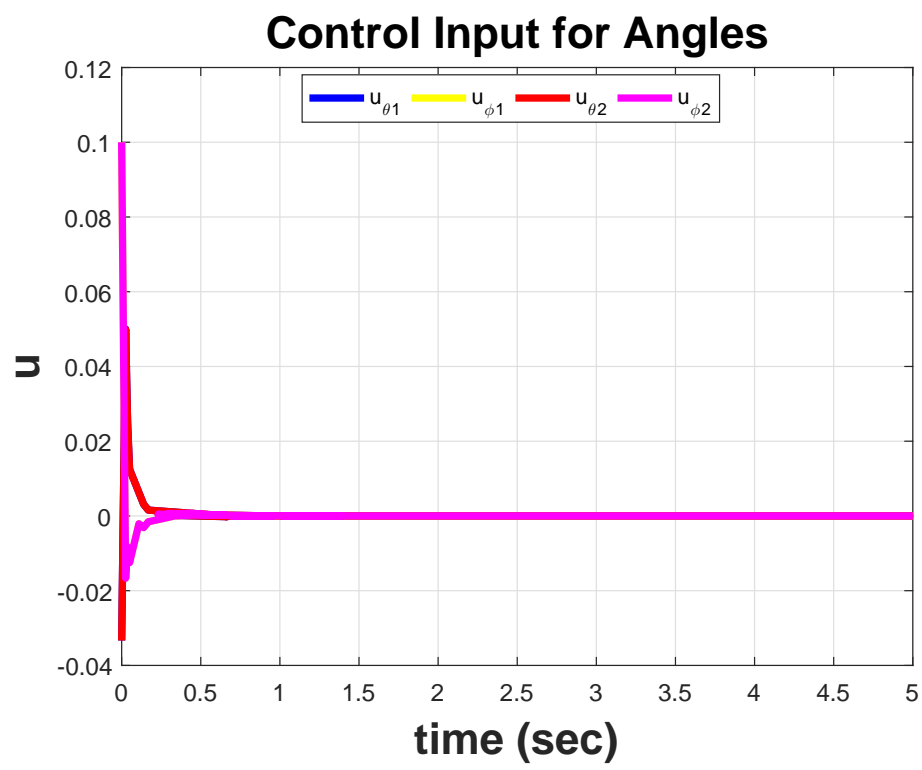

Figure 5.12: Control input for angles of two vehicles in 3D antenna orientated (NOESC-DS). 


\section{Vehicles Trajectory}

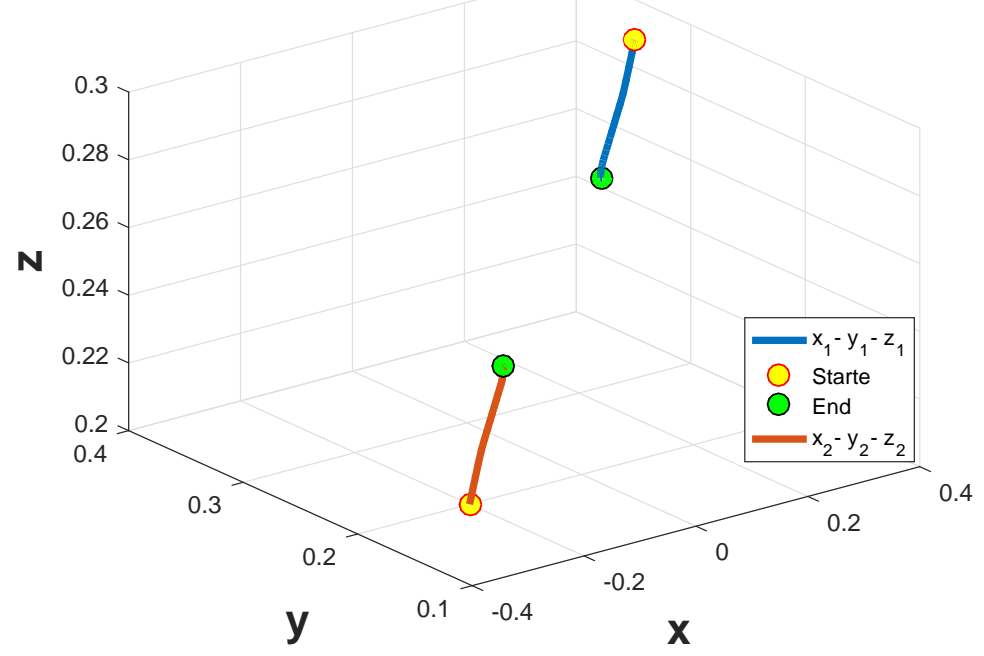

Figure 5.13: Vehicle trajectory for two vehicles in 3D antenna orientated (NOESC-DS).

\subsubsection{Using Numerical Optimization-based Extremum Seeking Control with PSO}

Using NOESC with PSO for 2 vehicles in $3 \mathbf{D}$, there are ten degrees of freedom because there are two vehicles each having a position of $(x, y, z)$ and orientation of $(\theta, \phi)$. Griddatan, which is a function that supports scattered data interpolation in N-D, produces $\hat{J}$. Only when using PSO, $\hat{J}$ is considered the objective function. The initial condition was put in as $x_{1}(0)=0.3, y_{1}(0)=0.3$, $z_{1}(0)=0.3 x_{2}(0)=-0.2, y_{2}(0)=0.2, z_{2}(0)=0.2, \theta_{1}(0)=0^{o}, \phi_{1}(0)=0^{o}, \theta_{2}(0)=0^{o}$, $\phi_{2}(0)=0^{\circ}$. The result for the objective function, which converged to 4.800 , are shown in Figure 5.14. As seen the objective function converged at about four seconds. Figure 5.15 shows the optimal position in this simulation converging to $\left(x_{1}^{*}, y_{1}^{*}, z_{1}^{*}\right)=(0.4145,0.1527,0.1563)$ and $\left(x_{2}^{*}, y_{2}^{*}, z_{2}^{*}\right)=(-0.2559,-0.0002,0.0719)$. Figure 5.16 shows the antenna orientation converging to the optimal angles of $\left(\theta_{1}^{*}, \phi_{1}^{*}\right)=\left(0.0011^{\circ}, 0.9911^{\circ}\right)$ and $\left(\theta_{2}^{*}, \phi_{2}^{*}\right)=\left(0.0225^{\circ}, 0.3217^{\circ}\right)$. Figure 5.17 shows that the control input for the position converged to a neighborhood of zero, and Figure 5.18 shows the control input for the angle orientation converged to a neighborhood of zero. 
It can be seen that the controller forced $\left(\theta_{1}, \phi_{1}\right)$ and $\left(\theta_{2}, \phi_{2}\right)$ to converge to zero. Figure 5.19, which is the vehicles trajectory, shows that the antenna position is arbitrary. The pink dots represent the initial condition, and the green dots represent the optimal position.

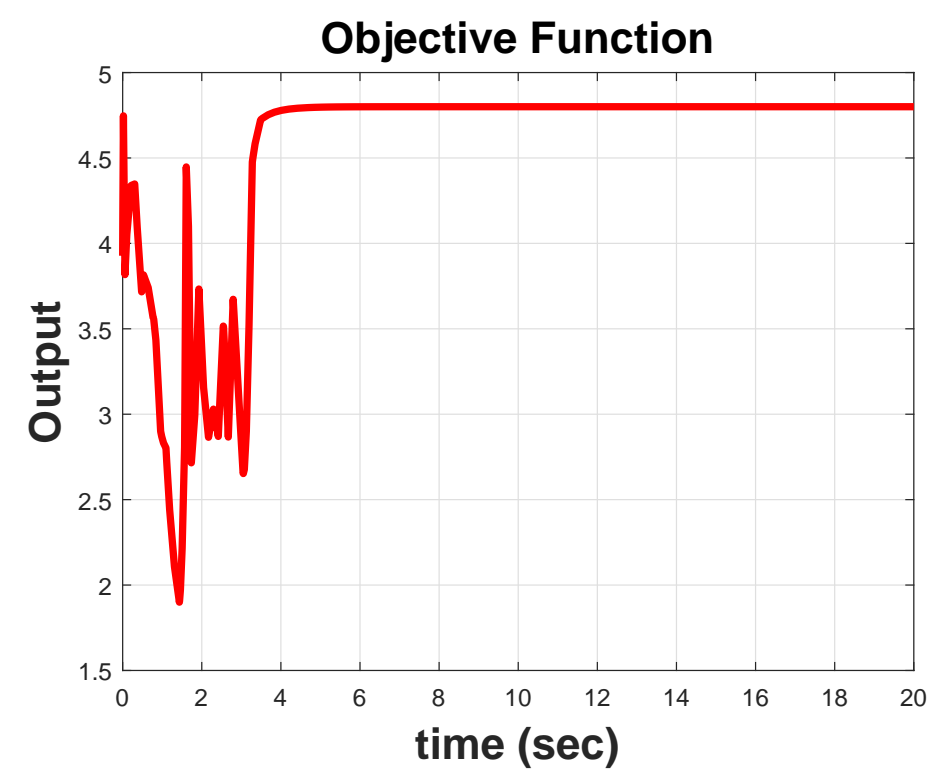

Figure 5.14: Objective function of two vehicles in 3D antenna orientated (NOESC-PSO). 


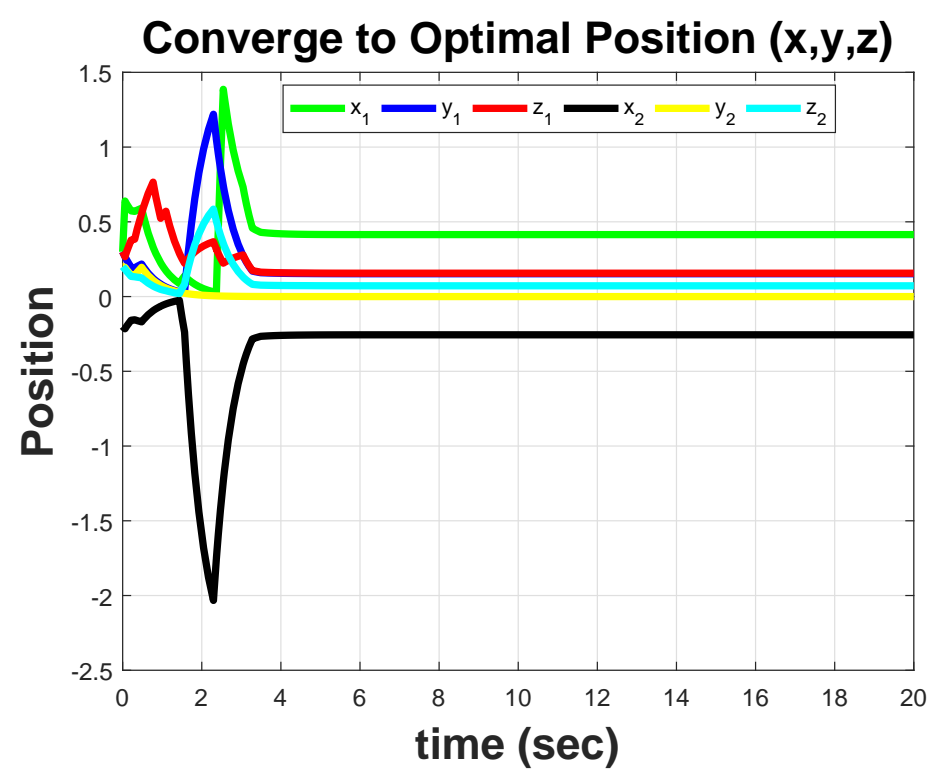

Figure 5.15: Optimal positions of two vehicles in 3D antenna orientated (NOESC-PSO).

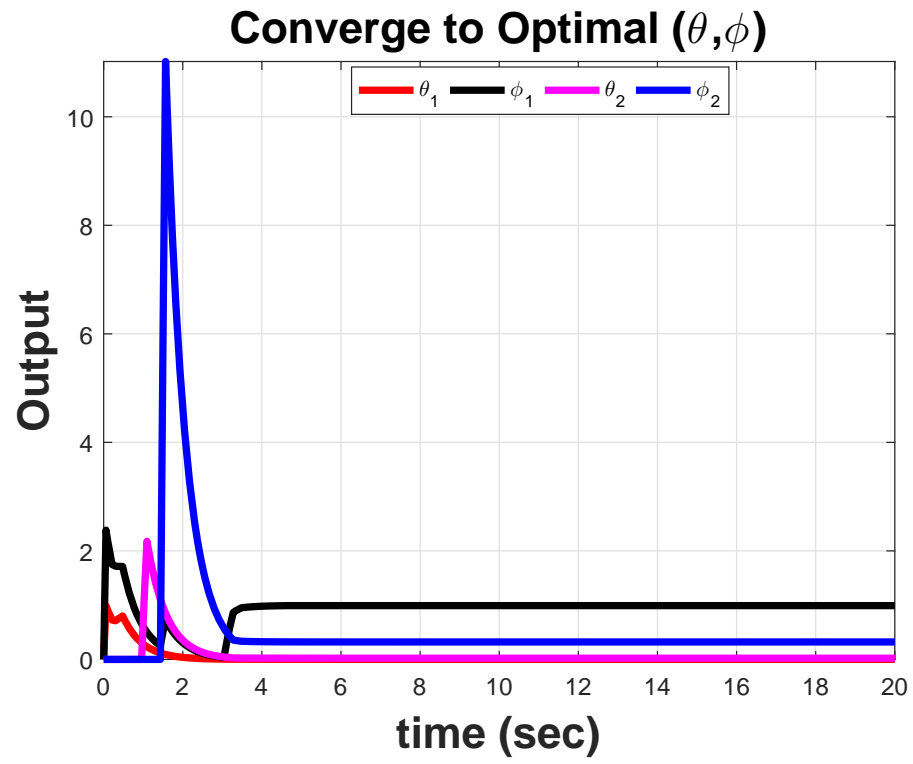

Figure 5.16: Optimal angles of two vehicles in 3D antenna orientated (NOESC-PSO). 


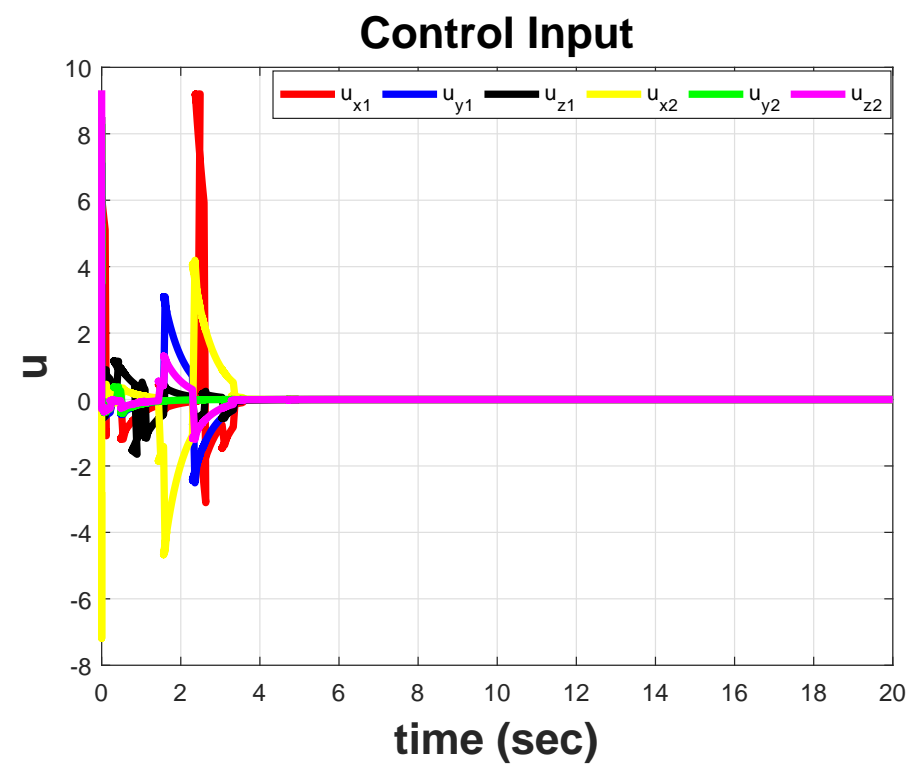

Figure 5.17: Control input for positions of two vehicles in 3D antenna orientated (NOESC-PSO).

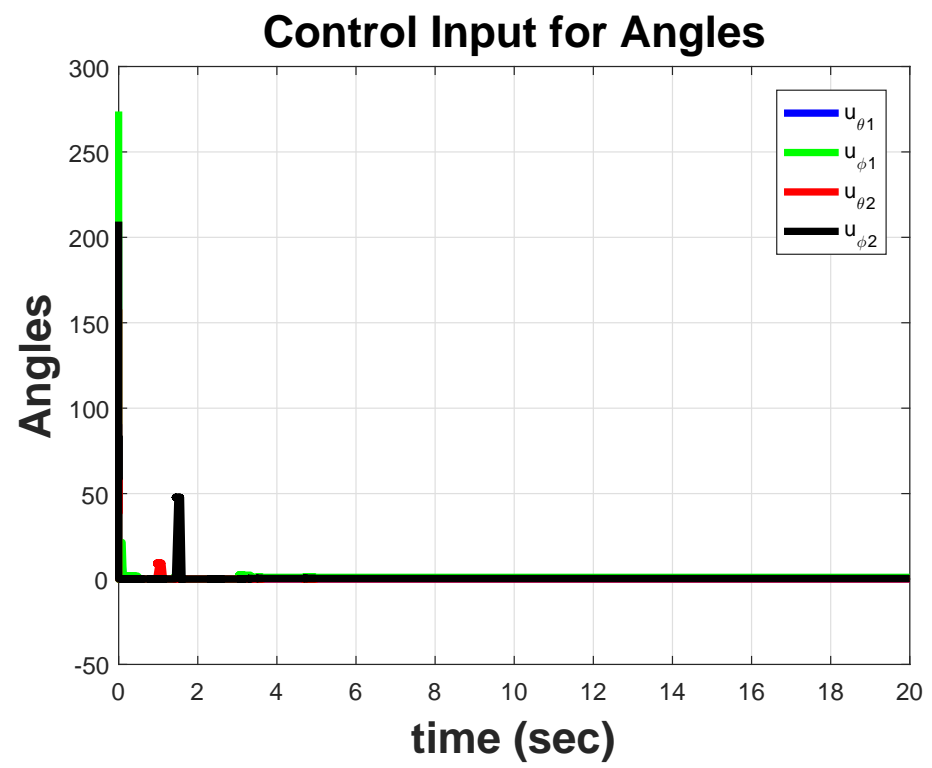

Figure 5.18: Control input for angles of two vehicles in 3D antenna orientated (NOESC-PSO). 


\section{Vehicles Trajectory}

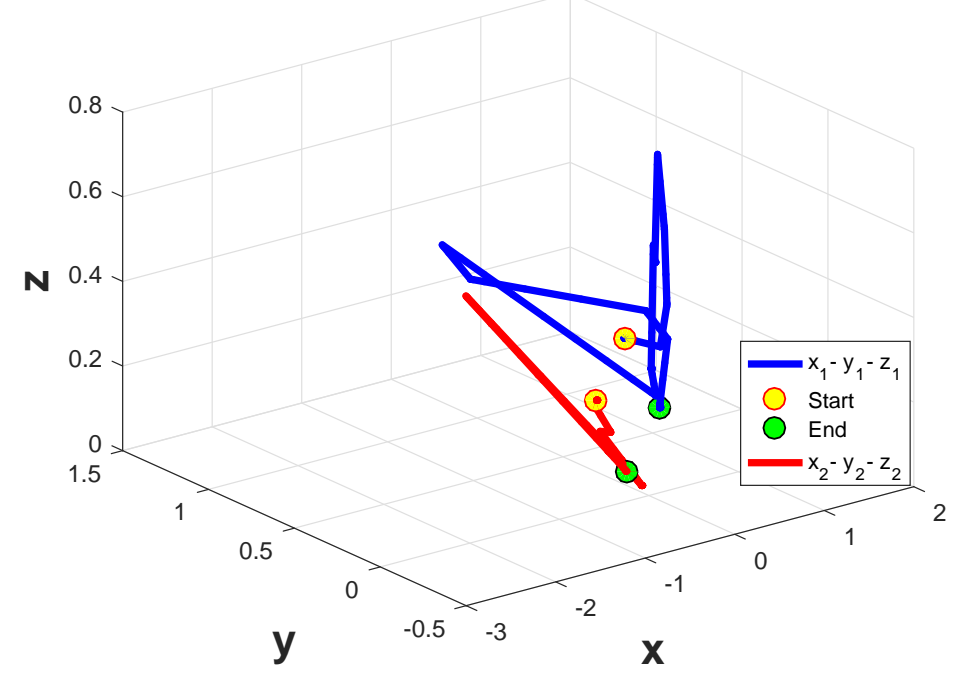

Figure 5.19: Vehicle trajectory for two vehicles in 3D antenna orientated (NOESC-PSO).

\subsubsection{Comparison of PESC and NOESC with DS and PSO for 2 Vehicles in 3D}

The three algorithms were able to get an optimal position and orientation. The NOESC-DS got better results than PESC and NOESC-PSO, and it gets the result faster in a simulation. The results aren't a unique solution, which means that if the intial condition is changed, it is possible to obtain an optimal solution, but with different positions.

\subsection{Four Vehicles in 3D with Arbitrary Antenna Orientation}

In this section we consider a three dimension case where $N$ vehicles are carrying directional orientation antennas. The $N$ vehicles will be studied from a four vehicle perspective. It will be tested using two algorithms, PESC and NOESC-PSO. We will study the five degrees of freedom, which are three degrees for the position $(x, y, z)$ and two degrees for the orientation $(\theta, \phi)$. The system should be able to test every possible value for these five parameters to dynamically choose the position and orientation that maximize the directivity in real time. 


\subsubsection{Using Perturbation-based Extremum Seeking Control}

Now again using the PESC algorithm in $3 \mathbf{D}$, we have four vehicles. This makes the system more complex. Each vehicle has five degrees of freedom, which means a total of 20 degree of freedom. We analyze how the system dynamically chooses the position and orientation that maximize the directivity. The simulation results can be found in Figures 5.20 - 5.22 where the initial conditions are $\left(x_{1}(0)\right)=0.81,\left(y_{1}(0)\right)=0.21,\left(z_{1}(0)\right)=1.25,\left(x_{2}(0)\right)=-0.43,\left(y_{2}(0)\right)=$ $0.31,\left(z_{2}(0)\right)=0.18,\left(x_{3}(0)\right)=-0.44,\left(y_{3}(0)\right)=-0.35,\left(z_{3}(0)\right)=0.15,\left(x_{4}(0)\right)=0.8$, $\left(y_{4}(0)\right)=-0.45$ and $\left(z_{4}(0)\right)=1.24$. And the initial condition for the antenna angle orientation are $\theta_{1}(0)=10^{\circ}, \phi_{1}(0)=10^{\circ}, \theta_{2}(0)=10^{\circ}, \phi_{2}(0)=10^{\circ}, \theta_{3}(0)=10^{\circ}, \phi_{3}(0)=10^{\circ}$, $\theta_{4}(0)=10^{\circ}$, and $\phi_{4}(0)=10^{\circ}$. The parameters of the perturbation based extremum seeking control simulation are selected as the perturbation frequency $\omega_{1}=21 \mathrm{rad} / \mathrm{s}, \omega_{2}=22 \mathrm{rad} / \mathrm{s}, \omega_{3}=23$ $\mathrm{rad} / \mathrm{s}, \omega_{4}=24 \mathrm{rad} / \mathrm{s}$, perturbation amplitude $\alpha_{i}=0.001$, adaptation gains $C_{x_{i}}=C_{y_{i}}=C_{z_{i}}=1$, and $i=1,2,3,4$. The performance function is increased to a neighborhood of the maximum. It can also be noticed that four different perturbation frequencies are needed for four vehicles in a three-dimensional space. The vehicle starts at the initial condition by probing around to climb the gradient of the unknown map. The output of the unknown signal $J$ is shown in Figure 5.20. In the same figure it shows that the PESC is indeed able to find the optimum directivity $J$ even though the optimum input varies with time. As expected, $J$ converge to a periodic motion around $J^{*}$. It also shows that the objective function was driven toward its optimum rapidly and kept at optimum by the gradient method. The noise that appears before the objective function reaches its optimum value of 11.6389 is caused by the fact that the Perturbationbased Extremum Seeking Control method is based on the sinusoidal wave perturbation. The PESC showed good results because it was able to find the optimal position for four vehicles, which is $\left(x_{1}^{*}, y_{1}^{*}, z_{1}^{*}\right)=(1.3316,0.0331,1.7716),\left(x_{2}^{*}, y_{2}^{*}, z_{2}^{*}\right)=(0.0691,0.1379,0.6791),\left(x_{3}^{*}, y_{3}^{*}, z_{3}^{*}\right)=$ 
$(0.0543,-0.5238,0.6443)$, and $\left(x_{4}^{*}, y_{4}^{*}, z_{4}^{*}\right)=(1.3136,-0.6254,1.7536)$, as seen in Figures 5.21

The orientation converged to $\left(\theta_{1}^{*}, \phi_{1}^{*}\right)=\left(10.5216^{\circ}, 9.8231^{\circ}\right),\left(\theta_{2}^{*}, \phi_{2}^{*}\right)=\left(10.4991^{\circ}, 9.8279^{\circ}\right)$, $\left(\theta_{3}^{*}, \phi_{3}^{*}\right)=\left(10.4943^{\circ}, 9.8262^{\circ}\right)$, and $\left(\theta_{4}^{*}, \phi_{4}^{*}\right)=\left(10.5136^{\circ}, 9.8246^{\circ}\right)$, as seen in Figure 5.22. The controller input for the position can be seen in Figure 5.23 , where it can be seen that it converges to a neighborhood of zero. And the controller input for the angles converges to a neighborhood of zero, which can be seen in Figure 5.24. The vehicles' trajectories are converging into the optimal position in 3D, and they are spread out into an arbitrary position as seen in Figure 5.25

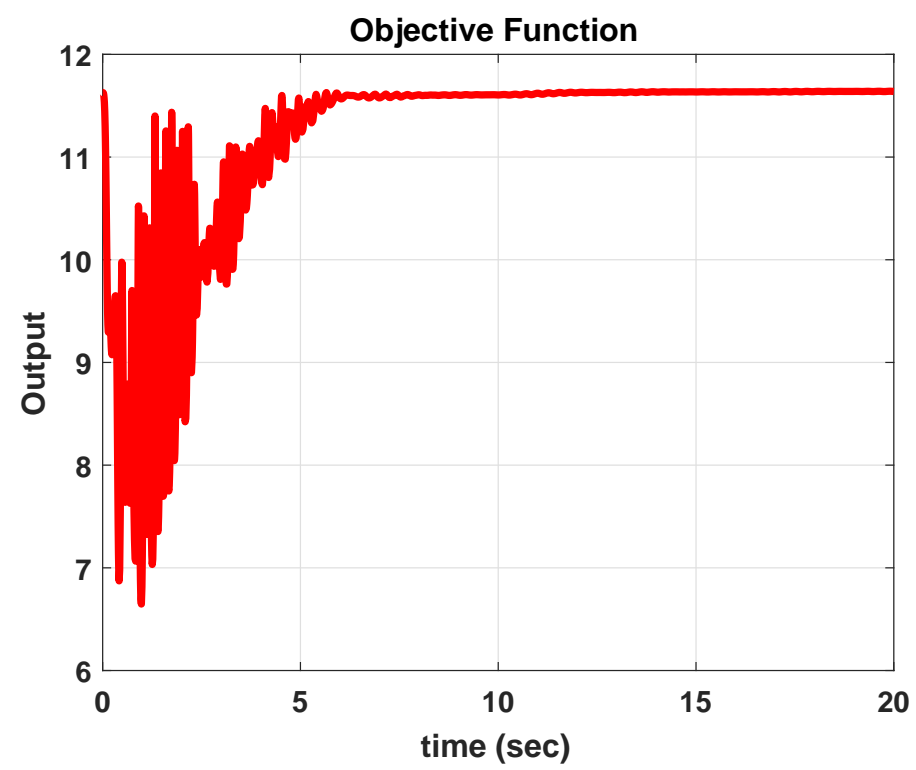

Figure 5.20: Objective function of four vehicles in 3D and antenna orientated (PESC). 


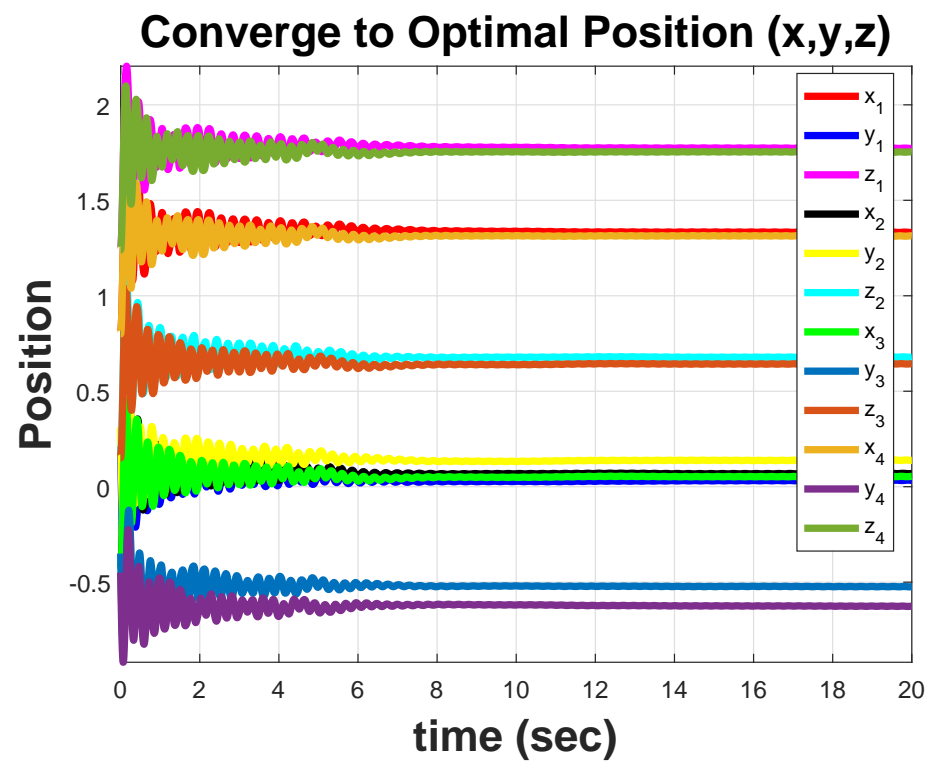

Figure 5.21: Optimal positions of four vehicles in 3D and antenna orientated (PESC).

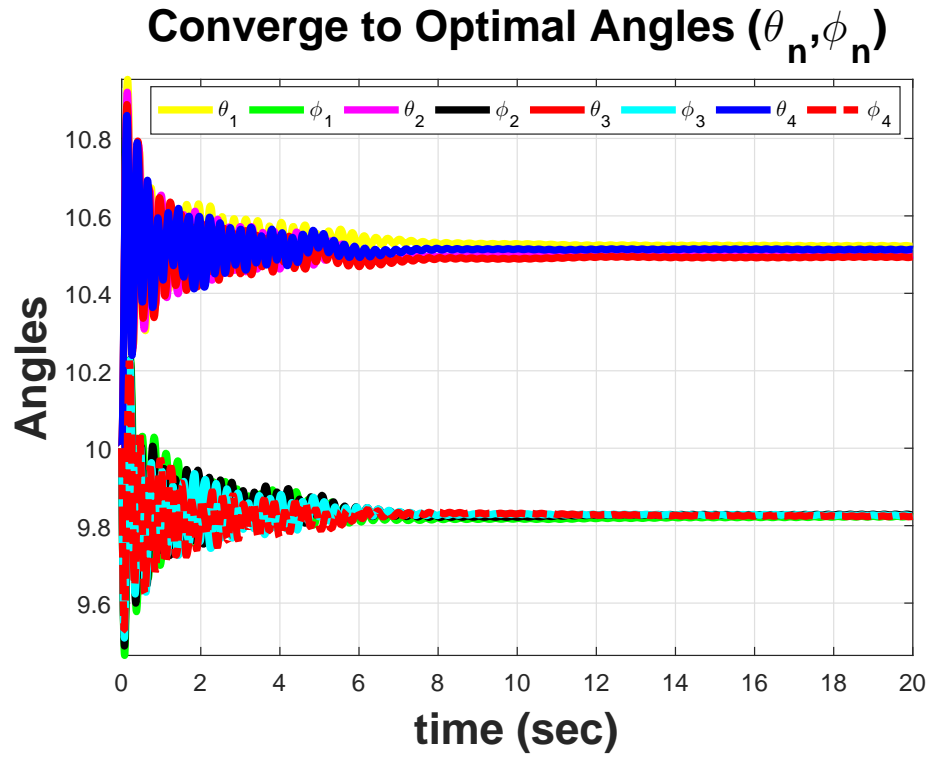

Figure 5.22: Optimal orientation angles for the antenna for four vehicles in 3D (PESC). 


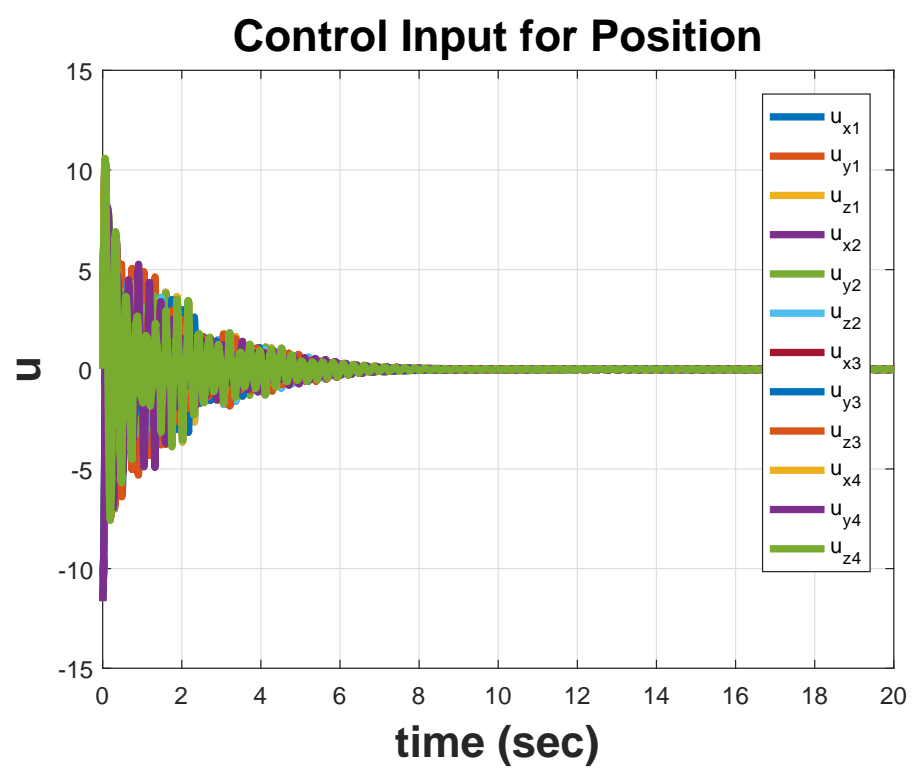

Figure 5.23: Control input for positions of four vehicles in 3D antenna orientated (PESC).

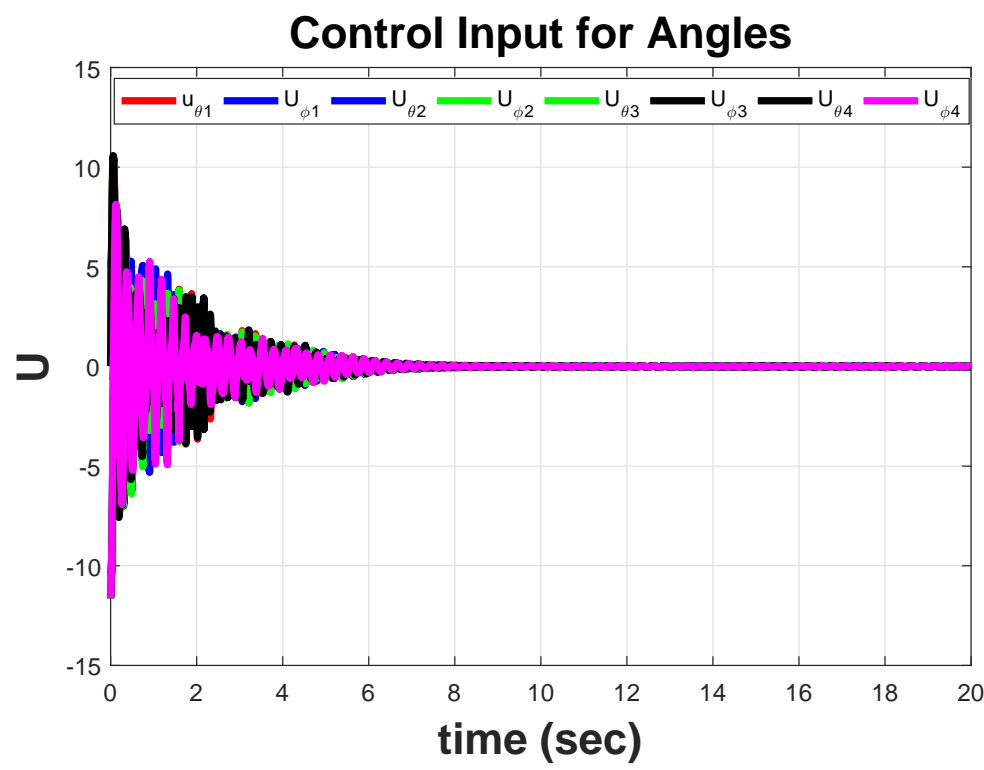

Figure 5.24: Control input for angles of four vehicles in 3D antenna orientated (PESC). 


\section{Vehicles Trajectory}

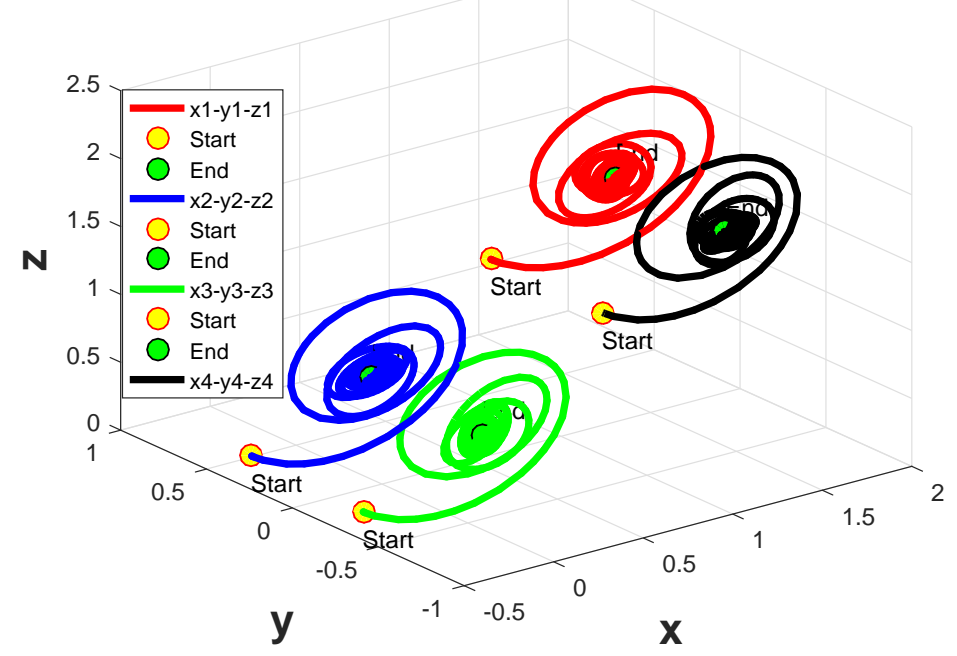

Figure 5.25: Vehicle trajectory for four vehicles in 3D antenna orientated (PESC).

\subsubsection{Using Numerical Optimization-based Extremum Seeking Control with DS}

Using NOESC with DS for 4 vehicles in $3 \mathbf{D}$, there is twenty degrees of freedom. The initial condition is the same as the ones used for PESC. The result for the objective function, which converged to 11.6639, are shown in Figure 5.26. As seen the objective function converged at about 0.25 seconds. Figure 5.27 shows the optimal position in this simulation converging to $\left(x_{1}^{*}, y_{1}^{*}, z_{1}^{*}\right)=$ $(0.8193,0.2123,1.2601),\left(x_{2}^{*}, y_{2}^{*}, z_{2}^{*}\right)=(-0.4283,0.3123,0.1699),\left(x_{3}^{*}, y_{3}^{*}, z_{3}^{*}\right)=$ $(-0.4461,-0.3523,0.1507)$, and $\left(x_{4}^{*}, y_{4}^{*}, z_{4}^{*}\right)=(0.8021,-0.4523,1.2453)$. The optimal antenna orientation angle, shown in Figure 5.28, is $\left(\theta_{1}^{*}, \phi_{1}^{*}\right)=\left(9.9772^{\circ}, 9.9772^{\circ}\right),\left(\theta_{2}^{*}, \phi_{2}^{*}\right)=$ $\left(9.9772^{\circ}, 9.9772^{\circ}\right),\left(\theta_{3}^{*}, \phi_{3}^{*}\right)=\left(9.9772^{\circ}, 9.9772^{\circ}\right)$, and $\left(\theta_{4}^{*}, \phi_{4}^{*}\right)=\left(9.9772^{\circ}, 9.9772^{\circ}\right)$. Figure 5.29 shows that the control input for the position converged to a neighborhood of zero, and Figure 5.30 shows the control input for the antenna orientation angles converged to a neighborhood of zero. Figure 5.31, which is the vehicles trajectory, shows that the antenna position is arbitrary. 


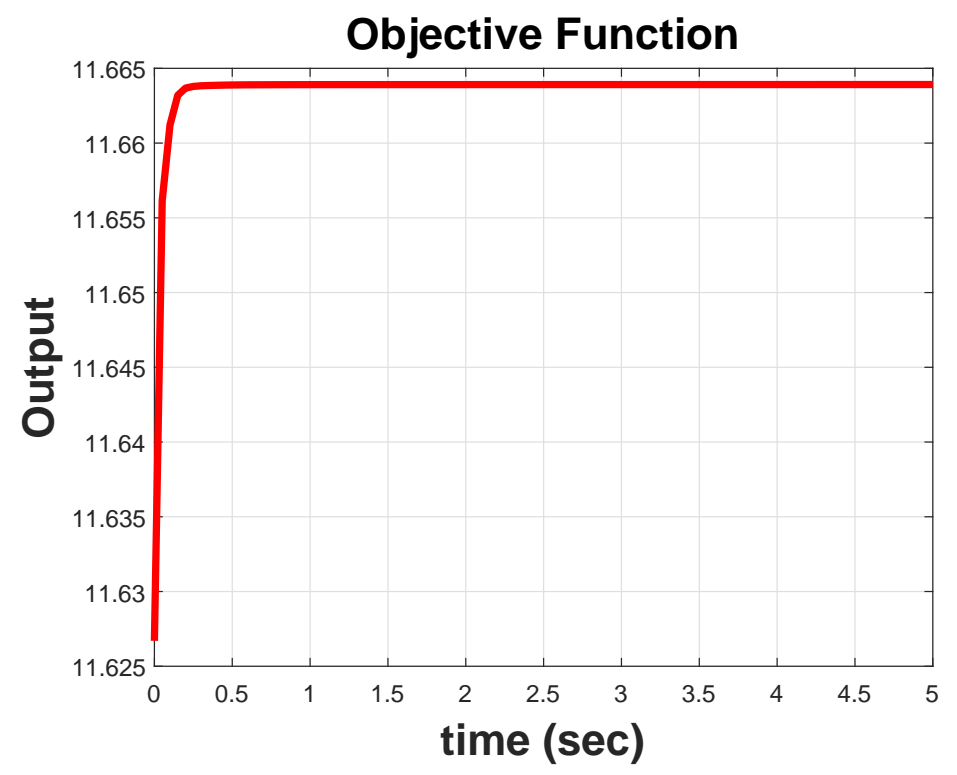

Figure 5.26: Objective function of four vehicles in 3D antenna orientated (NOESC-DS).

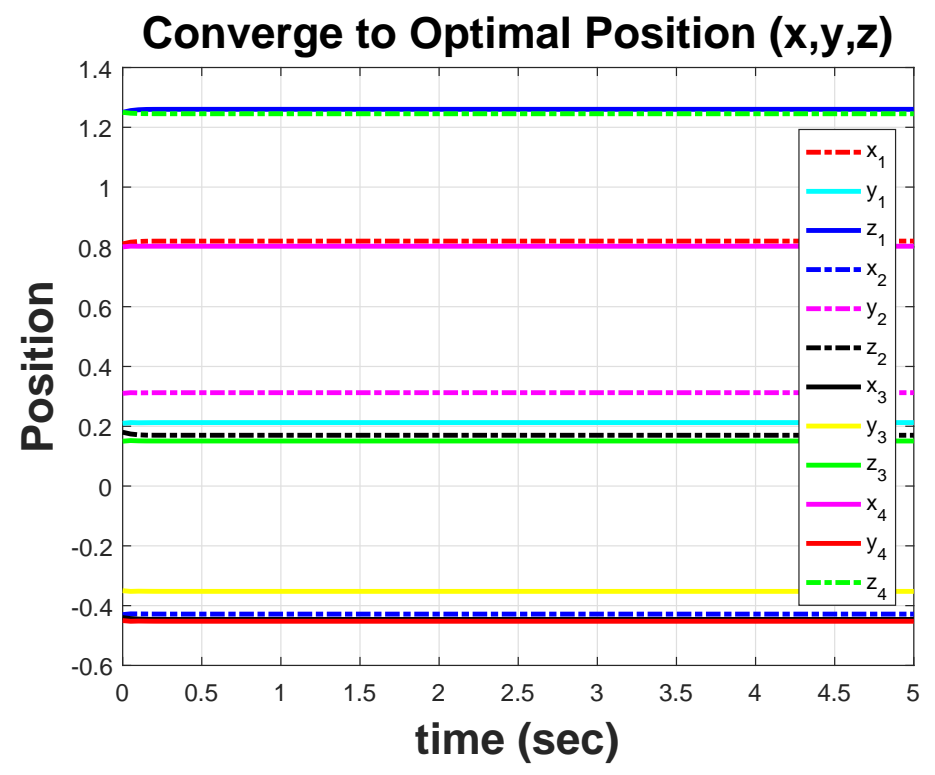

Figure 5.27: Optimal position of four vehicles in 3D antenna orientated (NOESC-DS). 


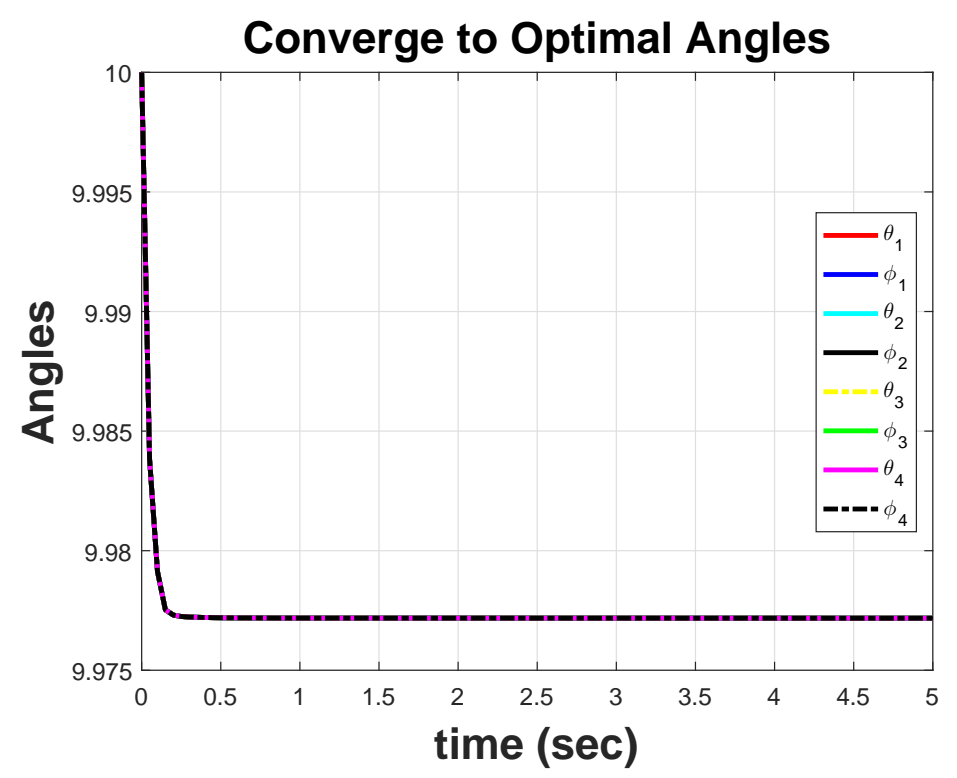

Figure 5.28: Optimal angle of four vehicles in 3D antenna orientated (NOESC-DS).

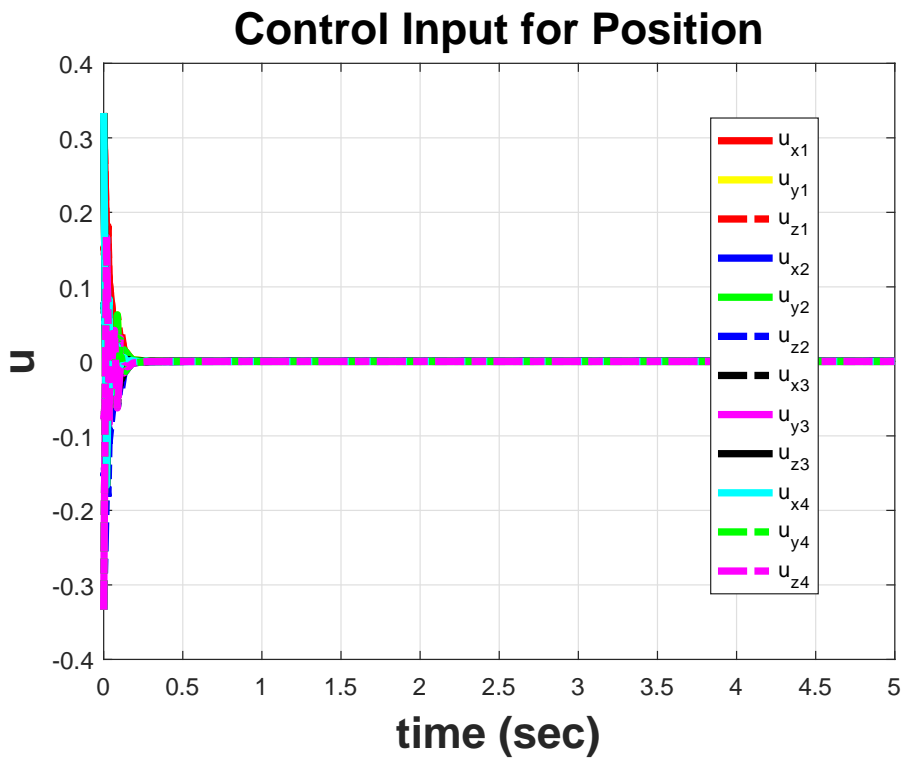

Figure 5.29: Control input for the position of four vehicles in 3D antenna orientated (NOESC-DS). 


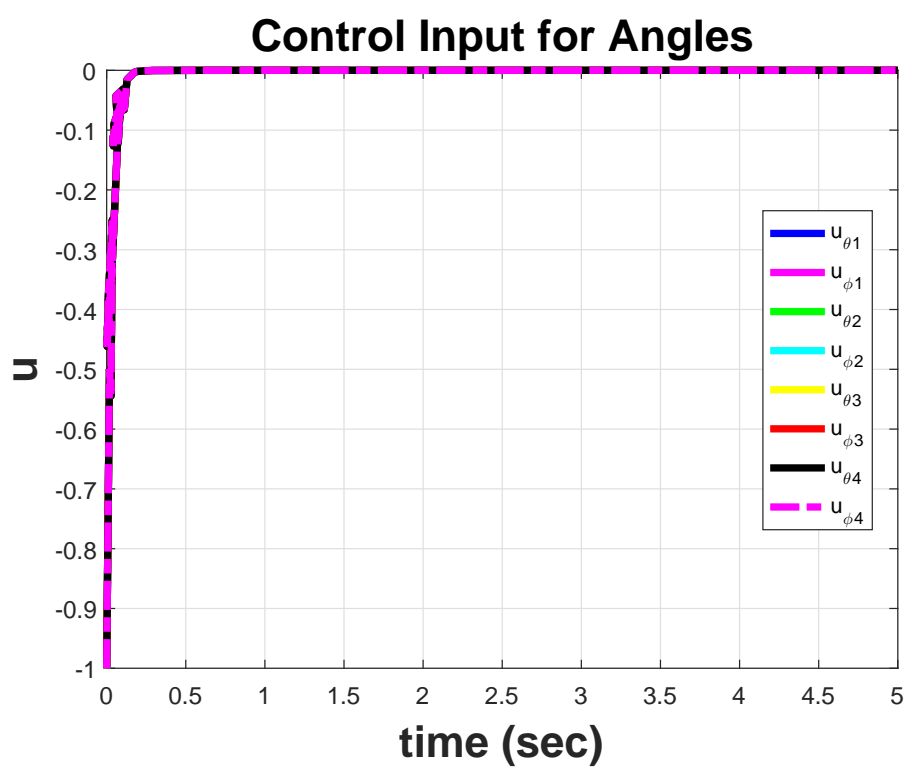

Figure 5.30: Control input for the angles of four vehicles in 3D antenna orientated (NOESC-DS).

\section{Converge to Optimal Position $(x, y, z)$}

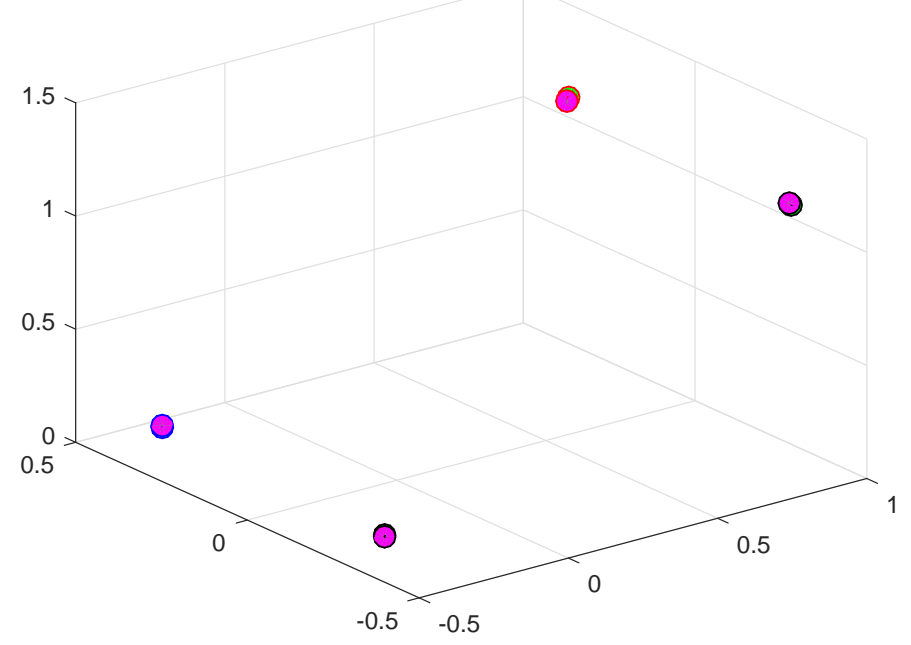

Figure 5.31: Vehicle trajectory for four vehicles in 3D antenna orientated (NOESC-DS). 


\subsubsection{Comparison of PESC and NOESC-DS for 4 Vehicles in 3D}

The two algorithms both got similar results for such a complex system. NOESC-DS got more desirable results because it converged to 11.66, which is higher than PESC's 11.6389. NOESC-DS converged faster at 0.25 seconds, and PESC converged at 6 seconds. This means overall NOESC-DS is slightly better. 


\section{CHAPTER VI}

\section{CONCLUSIONS AND FUTURE WORK}

\subsection{Conclusions}

We successfully designed a system that controls a non-uniform antenna array. This system was able to reconfigure the antenna array to find the position and orientation that produce the maximum objective (directivity).

Studying the sensitivity of the PESC or NOESC with PSO to changes in the initial condition in antenna array application showed evident that the PESC method and NOESC-PSO method are equally sensitive. When implementing the PESC and NOESC-PSO, they both converge or diverge to different optimal objective functions depending on the initial condition. With that it can be said that neither NOESC with PSO or PESC is more efficient than the other.

We assumed that we have $N$ vehicles carrying antennas. These vehicles were in $1 \mathbf{D}, 2 \mathbf{D}$, and 3D spaces (e.g., quad copters). Each vehicle with its antenna has five degrees of freedom. The five degrees are as follows, three degrees for the position of the vehicle $(x, y, z)$, and two for the orientation of the antenna $(\theta, \phi)$. The system was be able to test every possible value for these five parameters and dynamically choose the position and orientation that maximize the directivity. By measuring the performance function value online, a sequence, generated by three Extremum Seeking Control (ESC) algorithms that are compared in this work, guides the regulator that drives 
the state of system to a set point that optimizes the performance function. This approach develops a novel method to achieve reconfigurable antenna arrays, by defining an objective function to be optimized on-line using three different ESC methods: (a) Perturbation-based Extremum Seeking Control, (b) Numerical Optimization-based Extremum Seeking Control Using Direct Search, and (c) Numerical Optimization-based Extremum Seeking Control Using Particle Swarm Optimization.

In comparing the PESC, NOESC with Direct Search, and NOESC with PSO, it can be noticed that each algorithm performs the same task. The three algorithms got similar results for such an intricate system. The three algorithms were able to get an optimal position and orientation. Overall, NOESC-DS was able to get slightly better results out of all three algorithms. The three methods achieve the same final result, despite the fact that different algorithms were used to obtain the results.

We have found ESC to be a viable and effective method to achieve reconfigurable antenna arrays, capable of satisfying on the fly a given performance objective, even when the array elements can be moved in 3D space and the orientations of the antenna elements are allowed to vary. This is a challenging scenario, and our results indicate that it could be feasibly solved in practice using the proposed approach.

\subsection{Future Work}

To build off of what was done in this study, future work could include comparing the algorithms used in this dissertation with the DIRECT algorithm, to analyze differences and similarities. Also this study did not account for or look into collisions, so other works wanting to work upon this could see the effect of collision on the system. Obstacles were also one of the things that was not analyzed or focused on. Future works could see what problems obstacles could cause and how to solve any issues that may happen. Gaussian noise can also be added to the system to see what effects it could have. 


\section{BIBLIOGRAPHY}

[1] C. A. Balanis, Antenna theory: analysis and design. John Wiley \& Sons, 2005, vol. 1.

[2] W. L. Stutzman and G. A. Thiele, Antenna theory and design. John Wiley \& Sons, 2012.

[3] I. Wolff, "Determination of the radiating system which will produce a specified directional characteristic," Radio Engineers, Proceedings of the Institute of, vol. 25, no. 5, pp. 630-643, 1937.

[4] S. A. Schelkunoff, "A mathematical theory of linear arrays," Bell System Technical Journal, vol. 22, no. 1, pp. 80-107, 1943.

[5] C. Dolph, "A current distribution for broadside arrays which optimizes the relationship between beam width and side-lobe level," Proceedings of the IRE, vol. 34, no. 6, pp. 335-348, 1946.

[6] P. Woodward, "A method of calculating the field over a plane aperture required to produce a given polar diagram," Journal of the Institution of Electrical Engineers-Part IIIA: Radiolocation, vol. 93, no. 10, pp. 1554-1558, 1946.

[7] P. Woodward and J. Lawson, "The theoretical precision with which an arbitrary radiationpattern may be obtained from a source of finite size," Journal of the Institution of Electrical Engineers-Part III: Radio and Communication Engineering, vol. 95, no. 37, pp. 363-370, 1948.

[8] T. T. Taylor, "Design of line-source antennas for narrow beamwidth and low side lobes," Antennas and Propagation, Transactions of the IRE Professional Group on, vol. 3, no. 1, pp. 16-28, 1955.

[9] H. Unz, "Linear arrays with arbitrarily distributed elements," IRE Transactions on Antennas and Propagation, vol. 2, no. 8, pp. 222-223, 1960.

[10] J. Sahalos, "A solution of the general nonuniformly spaced antenna array," Proceedings of the IEEE, vol. 62, no. 9, pp. 1292-1294, 1974.

[11] H. Unz, "Nonuniformly spaced arrays: The orthogonal method," Proceedings of the IEEE, vol. 54, no. 1, pp. 53-54, 1966. 
[12] D. King, R. Packard, and R. Thomas, "Unequally-spaced, broad-band antenna arrays," Antennas and Propagation, IRE Transactions on, vol. 8, no. 4, pp. 380-384, 1960.

[13] R. F. Harrington, "Sidelobe reduction by nonuniform element spacing," Antennas and Propagation, IRE Transactions on, vol. 9, no. 2, pp. 187-192, 1961.

[14] Y. Lo, "A non-uniform linear array system for the radio telescope at the university of illinois," in 1960 Spring URSI and IRE Joint Meeting, Washington, DC, subsequently published in the IRE Trans. on Antennas and Propagation, vol. 9, 1961, pp. 9-16.

[15] J. Lan, R. K. Jeffers, and S. G. Boucher, "Optimum unequally spaced arrays and their amplitude shading," in Ultrasonics Symposium, 1995. Proceedings., 1995 IEEE, vol. 2. IEEE, 1995, pp. 965-969.

[16] R. L. Haupt, "Thinned arrays using genetic algorithms," Antennas and Propagation, IEEE Transactions on, vol. 42, no. 7, pp. 993-999, 1994.

[17] M. G. Bray, D. H. Werner, D. W. Boeringer, and D. W. Machuga, "Optimization of thinned aperiodic linear phased arrays using genetic algorithms to reduce grating lobes during scanning," Antennas and Propagation, IEEE Transactions on, vol. 50, no. 12, pp. 1732-1742, 2002.

[18] D. W. Boeringer and D. H. Werner, "Particle swarm optimization versus genetic algorithms for phased array synthesis," Antennas and Propagation, IEEE Transactions on, vol. 52, no. 3, pp. 771-779, 2004.

[19] B. Kumar and G. Branner, "Generalized analytical technique for the synthesis of unequally spaced arrays with linear, planar, cylindrical or spherical geometry," Antennas and Propagation, IEEE Transactions on, vol. 53, no. 2, pp. 621-634, 2005.

[20] M. S. Najjar and N. D. Khodier, "Design of non-uniform circular antenna arrays using particle swarm optimization," Journal of electrical engineering, vol. 59, no. 4, pp. 216-220, 2008.

[21] R. Abdolee, V. Vakilian, and T. A. Rahman, "Elements space and amplitude perturbation using genetic algorithm for antenna array side lobe cancellation," Signal Processing: An International Journal (SPIJ), vol. 2, no. 2, pp. 10-16, 2008.

[22] P. J. Bevelacqua, C. Balanis et al., "Geometry and weight optimization for minimizing sidelobes in wideband planar arrays," Antennas and Propagation, IEEE Transactions on, vol. 57, no. 4, pp. 1285-1289, 2009.

[23] M. Ridwan, M. Abdo, and E. Jorswieck, "Design of non-uniform antenna arrays using genetic algorithm," in Advanced Communication Technology (ICACT), 2011 13th International Conference on. IEEE, 2011, pp. 422-427.

[24] S. Rupcic, V. Mandric, and D. Zagar, "Reduction of sidelobes by nonuniform elements spacing of a spherical antenna array," Radioengineering, vol. 20, no. 1, pp. 299-306, 2011. 
[25] I. E. Lager, M. Simeoni, and C. Coman, "Mutual coupling in non-uniform array antennasan effective recipe," in Antennas and Propagation (EUCAP), 2012 6th European Conference on. IEEE, 2012, pp. 1518-1522.

[26] I. E. Lager and M. Simeoni, "Radiation properties of non-uniform array antennas," in 2012 42nd European Microwave Conference, 2012.

[27] C. Zhang and R. Ordóñez, Extremum-seeking control and applications: a numerical optimization-based approach. Springer Science \& Business Media, 2011.

[28] P. Blackman, "Extremum-seeking regulators," in An exposition of adaptive control. New York: Macmillan, 1962, pp. 36-50.

[29] C. S. Draper and Y. T. Li, Principles of optimalizing control systems and an application to the internal combustion engine. American Society of Mechanical Engineers, 1951.

[30] S. MEERKOV, "Asymptotic methods for investigating quasistationary states in continuous systems of automatic optimization(asymptotic methods for quasi-stationary modes in continuous automatic optimization systems, using averaging techniques)," AVTOMATIKA I TELEMEKHANIKA, pp. 119-139, 1967.

[31] S. Meerkov, "Asymptotic methods for investigating a class of forced states in extremal systems," Automation and Remote Control, vol. 12, no. 1916, p. 1920, 1967.

[32] O. Jacobs and G. Shering, "Design of a single-input sinusoidal-perturbation extremum-control system," in Proceedings of the Institution of Electrical Engineers, vol. 115, no. 1. IET, 1968, pp. 212-217.

[33] H. S. Tsien, Engineering cybernetics. McGraw-Hill book Company, Incorporated, 1954.

[34] A. Feldbaum, "Dual control theory," Automn. Remote Control, vol. 22, pp. 1-12, 1961.

[35] A. Krasovskii, "Dynamics of continuous self-tuning systems," Fizmatgiz, Moscow, 1963.

[36] D. J. Wilde, "Optimum seeking methods," 1964.

[37] E. P. I. Chinaev, “Self-tuning systems handbook," Automn. Remote Control, vol. 22, pp. 1-12, 1969.

[38] J. Sternby, "Extremum control systems: An area for adaptive control?" in Joint Automatic Control Conference, no. 17, 1980, p. 8.

[39] M. Krstić and H.-H. Wang, "Stability of extremum seeking feedback for general nonlinear dynamic systems," Automatica, vol. 36, no. 4, pp. 595-601, 2000.

[40] K. B. Ariyur and M. Krstic, Real-time optimization by extremum-seeking control. John Wiley \& Sons, 2003. 
[41] M. Krstić, "Performance improvement and limitations in extremum seeking control," Systems and Control Letters, vol. 39, no. 5, pp. 313-326, 2000.

[42] J.-Y. Choi, M. Krstić, K. B. Ariyur, and J. S. Lee, "Extremum seeking control for discrete-time systems," Automatic Control, IEEE Transactions on, vol. 47, no. 2, pp. 318-323, 2002.

[43] E. Schuster, C. Xu, N. Torres, E. Morinaga, C. Allen, and M. Krstic, "Beam matching adaptive control via extremum seeking," Nuclear Instruments and Methods in Physics Research Section A: Accelerators, Spectrometers, Detectors and Associated Equipment, vol. 581, no. 3, pp. 799815, 2007.

[44] Y. Ou, C. Xu, E. Schuster, T. Luce, J. Ferron, M. Walker, and D. Humphreys, "Design and simulation of extremum-seeking open-loop optimal control of current profile in the diii-d tokamak," Plasma Physics and Controlled Fusion, vol. 50, no. 11, p. 115001, 2008.

[45] J. Cochran, E. Kanso, S. D. Kelly, H. Xiong, and M. Krstic, "Source seeking for two nonholonomic models of fish locomotion," Robotics, IEEE Transactions on, vol. 25, no. 5, pp. 1166-1176, 2009.

[46] C. Zhang, D. Arnold, N. Ghods, A. Siranosian, and M. Krstic, "Source seeking with nonholonomic unicycle without position measurement and with tuning of forward velocity," Systems \& control letters, vol. 56, no. 3, pp. 245-252, 2007.

[47] A. Banaszuk, K. B. Ariyur, M. Krstić, and C. A. Jacobson, "An adaptive algorithm for control of combustion instability," Automatica, vol. 40, no. 11, pp. 1965-1972, 2004.

[48] H.-H. Wang, S. Yeung, and M. Krstić, "Experimental application of extremum seeking on an axial-flow compressor," Control Systems Technology, IEEE Transactions on, vol. 8, no. 2, pp. 300-309, 2000.

[49] H.-H. Wang and M. Krstić, "Extremum seeking for limit cycle minimization," Automatic Control, IEEE Transactions on, vol. 45, no. 12, pp. 2432-2436, 2000.

[50] M. S. Stanković and D. M. Stipanović, "Extremum seeking under stochastic noise and applications to mobile sensors," Automatica, vol. 46, no. 8, pp. 1243-1251, 2010.

[51] W. H. Moase, C. Manzie, and M. J. Brear, "Newton-like extremum-seeking for the control of thermoacoustic instability," Automatic Control, IEEE Transactions on, vol. 55, no. 9, pp. 2094-2105, 2010.

[52] P. M. Dower, P. M. Farrell, and D. Nešić, "Extremum seeking control of cascaded raman optical amplifiers," Control Systems Technology, IEEE Transactions on, vol. 16, no. 3, pp. 396-407, 2008.

[53] M. Guay and T. Zhang, "Adaptive extremum seeking control of nonlinear dynamic systems with parametric uncertainties," Automatica, vol. 39, no. 7, pp. 1283-1293, 2003. 
[54] C. Zhang and R. Ordonez, "Numerical optimization-based extremum seeking control with application to abs design," IEEE Transactions on Automatic Control, vol. 52, no. 3, pp. 454467, 2007.

[55] C. Zhang and R. Ordóñez, "Robust and adaptive design of numerical optimization-based extremum seeking control," Automatica, vol. 45, no. 3, pp. 634-646, 2009.

[56] C. Zhang and R. Ordónez, "Numerical optimization-based extremum seeking control of lti systems," in Proceedings of the 44th IEEE Conference on Decision and Control. IEEE, 2005, pp. 4428-4433.

[57] M. Hamza, "Extremum control of continuous systems," Automatic Control, IEEE Transactions on, vol. 11, no. 2, pp. 182-189, 1966.

[58] I. I. Ostrovskii, "Extremum regulation," Automatic and Remote Control, vol. 18, pp. 900-907, 1957.

[59] A. Pervozvanskii, "Continuous extremum control system in the presence of random noise," Automatic and Remote Control, vol. 21, pp. 673-677, 1960.

[60] R. C. Eberhart, J. Kennedy et al., "A new optimizer using particle swarm theory," in Proceedings of the sixth international symposium on micro machine and human science, vol. 1. New York, NY, 1995, pp. 39-43.

[61] M. Clerc and J. Kennedy, "The particle swarm-explosion, stability, and convergence in a multidimensional complex space," IEEE transactions on Evolutionary Computation, vol. 6, no. 1, pp. 58-73, 2002.

[62] K. E. Parsopoulos and M. N. Vrahatis, "Recent approaches to global optimization problems through particle swarm optimization," Natural computing, vol. 1, no. 2-3, pp. 235-306, 2002.

[63] A. P. Engelbrecht, "Fundamentals of computational swarm intelligence," 2005.

[64] T. Blackwell and J. Branke, "Multiswarms, exclusion, and anti-convergence in dynamic environments," IEEE transactions on evolutionary computation, vol. 10, no. 4, pp. 459-472, 2006.

[65] M. Pant, R. Thangaraj, and A. Abraham, "New particle swarm optimization algorithm incorporating reproduction operator for solving global optimization problems," in 7th International Conference on Hybrid Intelligent Systems (HIS 2007). IEEE, 2007, pp. 144-149.

[66] O. Djaneye-Boundjou, R. Ordónez, and V. Gazi, "Stable adaptive particle swarm optimization," ICCAS 2013, pp. 440-445, 2013. 